\title{
Robust Image Transmission with Rate- Compatible Low-Density Parity-Check codes over Noisy Channels
}

\author{
by \\ Xiang Pan \\ A thesis submitted to the \\ Faculty of Graduate Studies and Research \\ in partial fulfillment of the requirements for the degree of \\ Master of Applied Science in Electrical Engineering \\ Ottawa-Carleton Institute of Electrical and Computer Engineering \\ Department of Systems and Computer Engineering \\ Carleton University \\ Ottawa, Ontario \\ December, 2004 \\ (C) Copyright \\ Xiang Pan, 2004
}




$\begin{array}{ll}\begin{array}{l}\text { Library and } \\ \text { Archives Canada }\end{array} & \begin{array}{l}\text { Bibliothèque et } \\ \text { Archives Canada }\end{array} \\ \begin{array}{l}\text { Published Heritage } \\ \text { Branch }\end{array} & \begin{array}{l}\text { Direction du } \\ \text { Patrimoine de l'édition }\end{array} \\ \begin{array}{l}\text { 395 Wellington Street } \\ \text { Ottawa ON K1A ON4 }\end{array} & \begin{array}{l}\text { 395, rue Wellington } \\ \text { Ottawa ON K1A ON4 } \\ \text { Canada }\end{array}\end{array}$

Your file Votre référence

ISBN: 0-494-00751-6

Ourfile Notre référence

ISBN: 0-494-00751-6

NOTICE:

The author has granted a nonexclusive license allowing Library and Archives Canada to reproduce, publish, archive, preserve, conserve, communicate to the public by telecommunication or on the Internet, loan, distribute and sell theses worldwide, for commercial or noncommercial purposes, in microform, paper, electronic and/or any other formats.

The author retains copyright ownership and moral rights in this thesis. Neither the thesis nor substantial extracts from it may be printed or otherwise reproduced without the author's permission.
AVIS:

L'auteur a accordé une licence non exclusive permettant à la Bibliothèque et Archives Canada de reproduire, publier, archiver, sauvegarder, conserver, transmettre au public par télécommunication ou par l'Internet, prêter, distribuer et vendre des thèses partout dans le monde, à des fins commerciales ou autres, sur support microforme, papier, électronique et/ou autres formats.

L'auteur conserve la propriété du droit d'auteur et des droits moraux qui protège cette thèse. $\mathrm{Ni}$ la thèse ni des extraits substantiels de celle-ci ne doivent être imprimés ou autrement reproduits sans son autorisation.
In compliance with the Canadian

Privacy Act some supporting forms may have been removed from this thesis.

While these forms may be included in the document page count, their removal does not represent any loss of content from the thesis.
Conformément à la loi canadienne sur la protection de la vie privée, quelques formulaires secondaires ont été enlevés de cette thèse.

Bien que ces formulaires aient inclus dans la pagination, il n'y aura aucun contenu manquant.

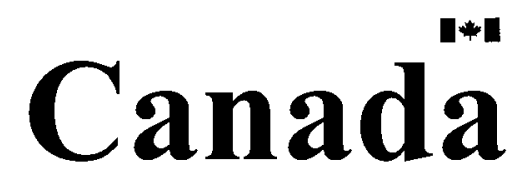




\begin{abstract}
Rate-Compatible Low-Density Parity-Check (RC-LDPC) codes are proposed to provide unequal error protection (UEP) for robust and efficient transmission of progressively compressed images over noisy channels. The total bit budget is partitioned between the source and channel coding, and the optimal rates are assigned to source encoded packets with a trellis-based rate-allocation algorithm using a forward error correction (FEC) scheme. We propose an improved rate-allocation algorithm by which the speed could be increased up to about three orders of magnitude due to the reduction in the complexity of the trellis. Simulation results based on Joint Photographic Experts Group (JPEG) 2000 and set partitioning in hierarchical trees (SPIHT) source encoders show that the proposed FEC schemes over binary symmetric channel (BSC) outperforms similar schemes based on turbo codes and irregular repeat-accumulate codes by up to about $1.1 \mathrm{~dB}$ and $1 \mathrm{~dB}$ in the expected peak signal-to-noise ratio (PSNR) of reconstructed images. Moreover, the proposed FEC scheme achieves up to about $1.7 \mathrm{~dB}$ and 3 $\mathrm{dB}$ improvements over EEP and UEP with product codes respectively over Rayleigh fading channel. For applications where delay is not a major concern, we present an alternative UEP scheme using automatic repeat request (ARQ), where parity check bits are incrementally appended until source packets are correctly recovered.
\end{abstract}




\section{Acknowledgements}

I would like to give my deep gratitude to my thesis Co-supervisors Professor Aysegul Cuhadar and Professor Amir H. Banihashemi for their careful guidance, encouragement and enthusiasm in my research work. Their patience, critical insight and helpful suggestions were invaluable in the completion of this work.

I would also like to thank Mohammad-Reza Yazdani for his sincere help on my research. And all my friends and colleagues at our research group and laboratory helped me a lot by providing an intellectually stimulating and enjoyable atmosphere. 


\section{Table of Contents}

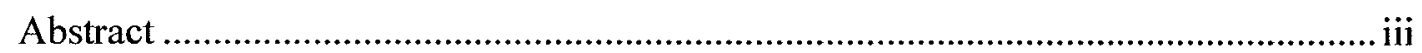

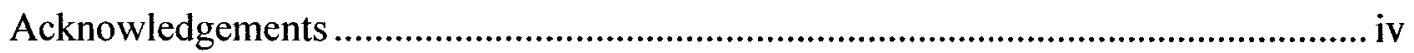

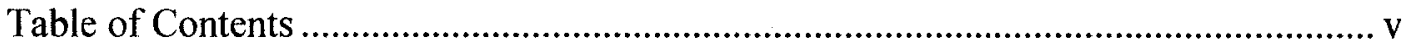

List of Tables...................................................................................................... vii

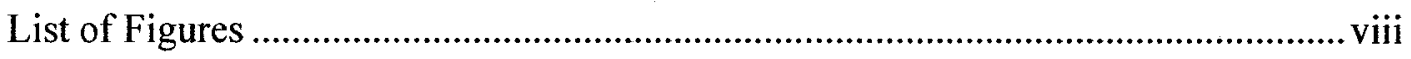

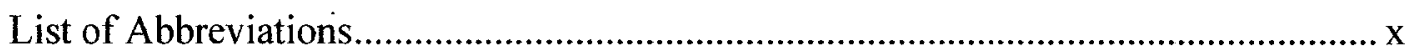

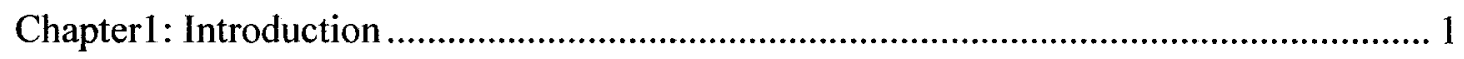

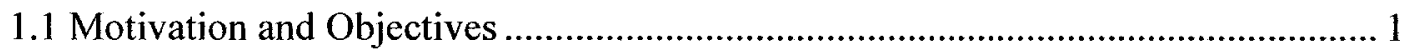

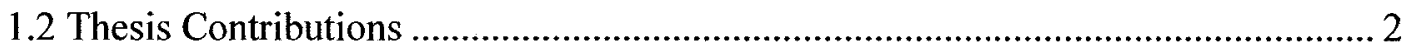

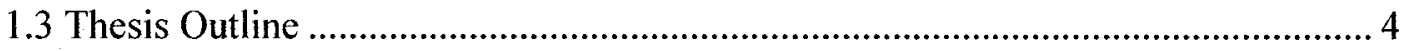

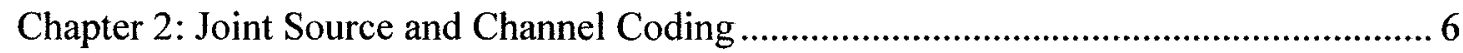

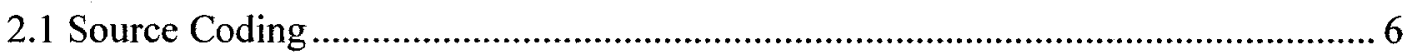

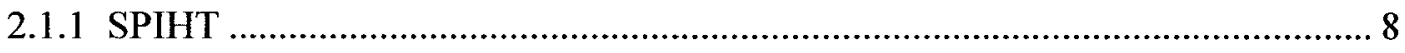

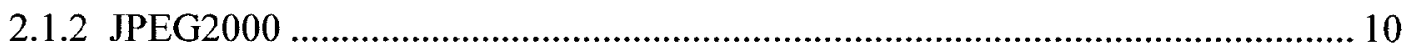

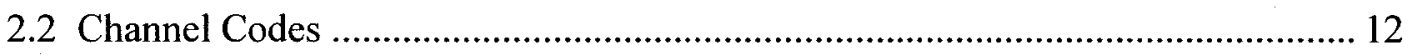

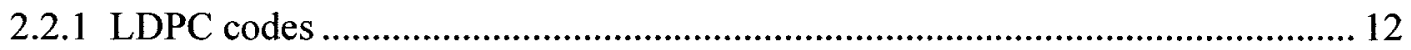

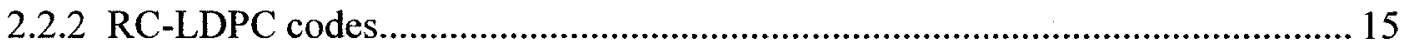

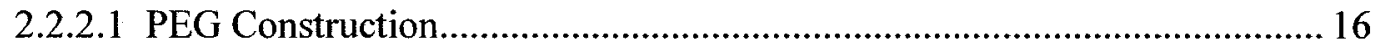

2.2.2.2 Encoding and Decoding of LDPC codes................................................. 16

2.2.2.3 General Construction of RC-LDPC codes............................18

Chapter 3 Rate-Allocation Algorithm.............................................20

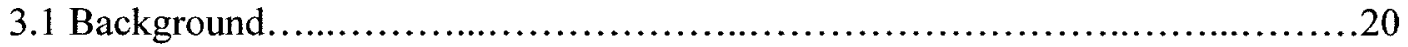


3.3 Performance of the Fast Trellis-Based Rate-Allocation Algorithm.................... 31

Chapter 4: Joint Source and Channel Coding with over BSC with FEC ......................... 36

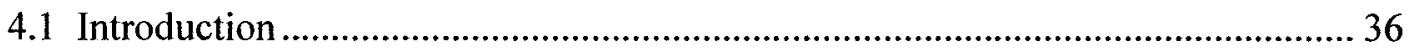

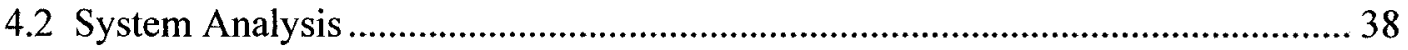

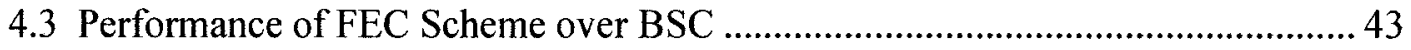

4.4 Estimation Error for Chanel Parameter ................................................................ 48

Chapter 5: Progressive Image Transmission over Fading Channel with FEC.................. 50

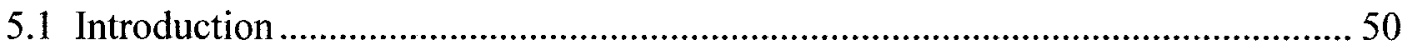

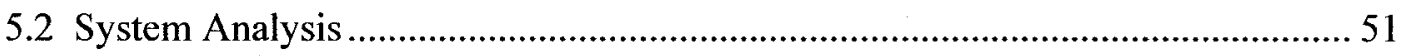

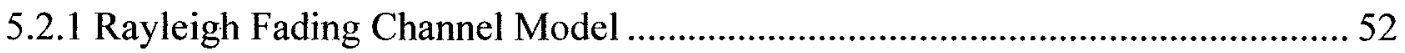

5.3 Performance of FEC Scheme over Rayleigh Fading Channels............................ 53

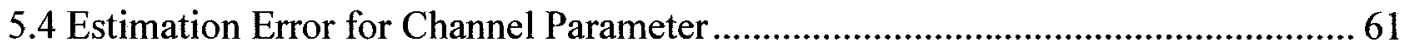

Chapter 6: Progressive Image Transmission over Noisy Channels with ARQ ...............68

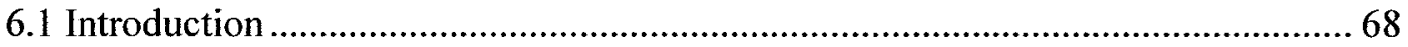

6.2 System Analysis.......................................................69

6.3 Performance of ARQ Scheme over BSC …................................................... 71

6.4 Performance of ARQ Scheme over Rayleigh Flat-Fading Channels .................... 74

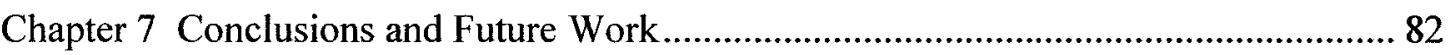

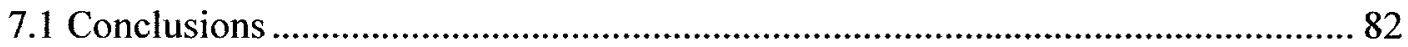

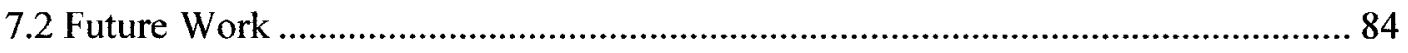

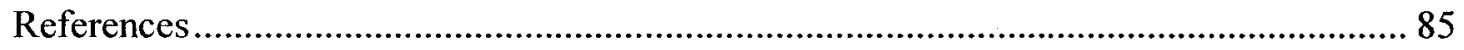

$\mathrm{vi}$ 


\section{List of Tables}

Table 3.1: Speed-up factors of the proposed rate-allocation algorithm compared with that of [2]. 34

Table 4.1: Expected PSNR (dB) for RC-LDPC codes, RCTCs and IRA Codes..........46

Table 4.2. Expected PSNR (dB) for the proposed schemes based on RC-LDPC codes RCTCs and IRAs, and for the error-free transmission at capacity rate (Total rate = 0.994 bpp).

Table 4.3. Statistics (mean and standard deviation) of the number of iterations required for the convegence of each RC-LDPC coded packet .............................................. 48

Table 4.4. Expected PSNR (dB) with mismatched BERs (Total rate $=0.994 \mathrm{bpp}) \ldots \ldots \ldots . . .48$ 


\section{List of Figures}

Figure 2.1. Illustration of 2-level wavelet transform of "Lena" into sub-bands.................. 8

Figure 2.2. Illustration of the parent-child relation for the coefficients of SPIHT ........... 9

Figure 2.3. Tanner graph of LDPC codes ................................................................ 13

Figure 2.4. General Construction of the parity-check matrices for the mother code and extendedcodes in the sequence of RC-LDPC codes ........................................ 18

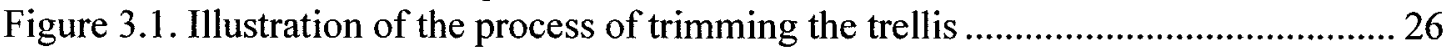

Figure 3.2. The WER curves of the channel codes over a BSC channel........................ 32

Figure 3.3. Optimal ratio of source bit budget to total bit budget for JPEG2000 algorithm.

Figure 3.4. Average optimal ratio of source bit budget to total bit budget for JPEG2000

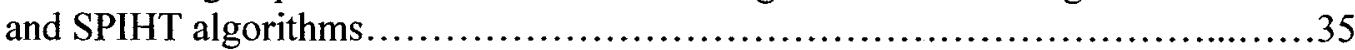

Figure 4.1. Block Diagram for FEC Scheme.................................... 38

Figure 4.2.(a) PSNR vs. information block length ' $k$ ' for 'Lena", when total bit budget

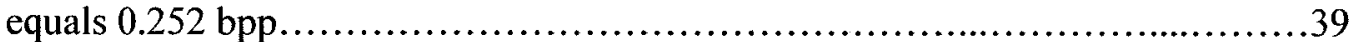

Figure 4.2.(b) PSNR vs. information block length ' $k$ ' for 'Lena", when total bit budget equals 0.505 bpp............................................................40

Figure 4.3. Construction of the parity-check matrices for the mother code and extended codes in the sequence of RC-LDPC codes..

Figure 4.4. Performance of RC-LDPC codes with information block length $\mathrm{K}=384$ bytes at different rates (solid lines); Performance of RCTCs of [2] with block length $=$

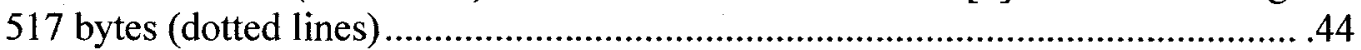

Figure 5.1. Rayleigh fading channel model .............................................................. 52

Figure 5.2.(a) WER curves of RC-LDPC codes for different Rayleigh fading channels; K $=384$ bytes; Uncorrelated Rayleigh fading channel with SI ................................. 54

Figure 5.2.(b) WER curves of RC-LDPC codes for different Rayleigh fading channels; K $=384$ bytes; Uncorrelated Rayleigh fading channel without SI............................ 55

Figure 5.2.(c) WER curves of RC-LDPC codes for different Rayleigh fading channels; $\mathrm{K}$ $=384$ bytes; Correlated Rayleigh fading channel; Vehicle speed V $=4 \mathrm{~m} / \mathrm{h} ; \ldots \ldots . . .55$

Figure 5.2.(d) WER curves of RC-LDPC codes for different Rayleigh fading channels; K $=384$ bytes; Correlated Rayleigh fading channel; Vehicle speed $\mathrm{V}=30 \mathrm{~m} / \mathrm{h} \ldots \ldots . .56$

Figure 5.2.(e) WER curves of RC-LDPC codes for different Rayleigh fading channels; $\mathrm{K}=384$ bytes; Correlated Rayleigh fading channel; Vehicle speed $\mathrm{V}=70 \mathrm{~m} / \mathrm{h} . . .56$

Figure 5.3.(a) End-to-end PSNRs for JPEG2000 encoded 512×512, 8 bit monochrome "Lena" image over different Rayleigh fading channels; Including the PSNRs for underestimation and over-estimation assuming the source image is encoded for the channel; PSNRs for uncorrelated Rayleigh fading channel with SI compared with

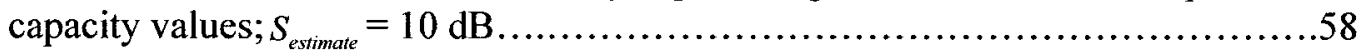

Figure 5.3.(b) End-to-end PSNRs for JPEG2000 encoded 512×512, 8 bit monochrome "Lena" image over different Rayleigh fading channels; Including the PSNRs for underestimation and over-estimation assuming the source image is encoded for the channel; PSNRs for uncorrelated Rayleigh fading channel without SI compared

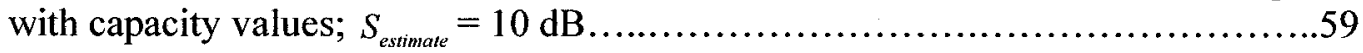


Figure 5.3.(c) End-to-end PSNRs for JPEG2000 encoded 512×512, 8 bit monochrome "Lena" image over different Rayleigh fading channels; Including the PSNRs for underestimation and over-estimation assuming the source image is encoded for the channel; PSNRs for correlated Rayleigh fading channel; Vehicle speed V=4 m/h; $S_{\text {estimate }}=15 \mathrm{~dB}$.

Figure 5.3.(d) End-to-end PSNRs for JPEG2000 encoded 512×512, 8 bit monochrome "Lena" image over different Rayleigh fading channels; Including the PSNRs for underestimation and over-estimation assuming the source image is encoded for the channel; PSNRs for correlated Rayleigh fading channel; Vehicle speed V $=30 \mathrm{~m} / \mathrm{h}$;

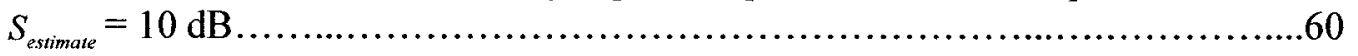

Figure 5.3.(e) End-to-end PSNRs for JPEG2000 encoded $512 \times 512,8$ bit monochrome "Lena" image over different Rayleigh fading channels; Including the PSNRs for underestimation and over-estimation assuming the source image is encoded for the channel; PSNRs for correlated Rayleigh fading channel; Vehicle speed $V=70 \mathrm{~m} / \mathrm{h}$;

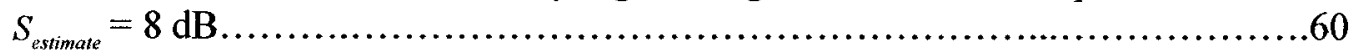

Figure 5.4. End-to-end PSNRs for SPIHT encoded "Lena" image for correlated Rayleigh fading channels with different normalized Doppler spread from $1 \times 10^{-5}$ to $2 \times 10^{-4}$ $(\mathrm{SNR}=13 \mathrm{~dB}$, total transmission rate $=0.25 \mathrm{bpp})$

Figure 5.5. End-to-end PSNRs for SPIHT encoded "Lena" image for correlated Rayleigh fading channels with normalized Doppler spread of $1 \times 10^{-4}$ (the total transmission rate is $0.25 \mathrm{bpp}$ )

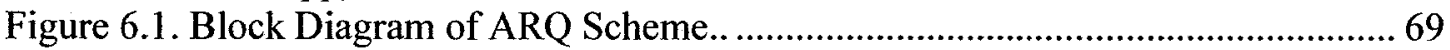

Figure 6.2. The throughput for type-II hybrid-ARQ scheme compared to the channel ca-

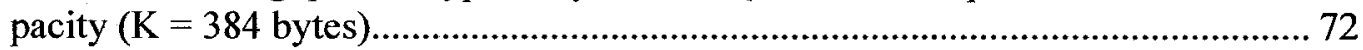

Figure 6.3.(a) Performance of FEC and hybrid-ARQ scheme $(\mathrm{K}=384$ bytes); "Lena".. 73

Figure 6.3.(b) Performance of FEC and hybrid-ARQ scheme $(K=384$ bytes); "Goldhill".

Figure 6.4. Throughput of Rayleigh flat-fading channel with SI, compared with channelcapacity

Figure 6.5. The PSNRs of FEC and ARQ schemes for uncorrelated Rayleigh flat-fading channel with SI compared with channel capacity values; for "Lena". 76

Figure 6.6. Throughput of Rayleigh flat-fading channel without SI compared with channel capacity

Figure 6.7. The PSNRs of FEC and ARQ schemes for uncorrelated Rayleigh flat-fading channel without SI compared with channel capacity values.. 78

Figure 6.8.(a) The PSNRs of JPEG-2000 coded "Lena" for FEC and ARQ schemes transmitted over correlated Rayleigh fading channels; The mobile speed $V=4 \mathrm{~m} / \mathrm{h}$

Figure 6.8.(b) The PSNRs of JPEG-2000 coded "Lena" for FEC and ARQ schemes transmitted over correlated Rayleigh fading channels; The mobile speed $\mathrm{V}=30$ $\mathrm{m} / \mathrm{h}$. 80

Figure 6.8.(c) The PSNRs of JPEG-2000 coded "Lena" for FEC and ARQ schemes transmitted over correlated Rayleigh fading channels; The mobile speed $\mathrm{V}=70$ $\mathrm{m} / \mathrm{h}$. 


\section{List of Abbreviations}

\begin{tabular}{ll} 
Abbreviations & \\
BP & Belief Propagation \\
bpp & bit per pixel \\
CRC & Cyclic Redundancy Check \\
DWT & Discrete Wavelet Transformation \\
EBCOT & Embedded Wavelet Coding with Optimal Truncation \\
EEP & Equal Error Protection \\
EZW & Embedded Zero-tree Wavelet \\
FEC & Forward Error Correction \\
IRA & Irregular Repeat Accumulate \\
JPEG & Joint Photographic Experts Group \\
JSCC & Point Source and Channel Coding \\
LIP & Progressive Edge Growth \\
LIS & List of Insignificant Pixels \\
LSP & List of Insignificant Sets \\
MSE & List of Significant Pixels \\
NACK & Mean Squared Error \\
PCRC & Post-Compression Rate-Control \\
\hline
\end{tabular}


RC-LDPC Rate-Compatible Low-Density Parity-Check Codes

RCPC Rate-Compatible Punctured Convolutional codes

RCTC Rate-Compatible Turbo Codes

SI

Side Information

SNR

Signal to Noise Ratio

SPIHT

Set Partitioning in Hierarchical Trees

UEP

Unequal Error Protection

WER

Word Error Rate

$\mathrm{xi}$ 


\section{Chapter 1}

\section{Introduction}

\subsection{Motivation and Objectives}

Transmission of images/video over internet and wireless communication channels requires robust and efficient source and channel coding algorithms. The current image coding standard JPEG2000 or SPIHT algorithm provides progressive image compression where the original image can be reconstructed incrementally. The main drawback of progressive organization of the bitstream is that it is highly prone to transmission noise. Channel codes are required to protect the source encoded bitstream. Traditionally, the problems of source coding and channel coding have been addressed independently. However, when the constraints of the communication channel are considered, a joint source/channel coding scheme (JSCC) is found to be the most promising scheme for communication of images over noisy channels. In particular, rate-compatible (RC) channel codes, due to their ability to cope with varying channel conditions are found to perform close to the theoretical bounds of transmission rates. Different RC channel codes for implementing joint source and channel coding have been studied, including RC punctured convolutional codes (RCPC), Reed-Solomon codes, RC turbo codes (RCTC) and 
irregular repeat accumulate (IRA) codes. It is known that RC-LDPC codes provide nearShannon limit error correction performance [1]. In this work we use them in the context of JSCC over noisy channels and show that JSCC with RC-LDPC codes is promising.

The main objective of this thesis is to provide solutions to the problem of progressive transmission of images over BSC or Rayleigh flat-fading channels using RC-LDPC codes. We propose a framework where we determine the optimal channel code rates to maximize the end-to-end peak signal to noise ratio (PSNR) (which is the PSNR between the source image and the recovered one). Also, we present a rate-allocation algorithm for a FEC system to minimize the distortion at high speed. Furthermore, we examine the performance of the proposed scheme for an ARQ channel model using RC-LDPC codes. In our simulations we use the current image coding standard JPEG2000 and SPIHT image coding algorithms to study the performance of the proposed JSCC scheme using RCLDPC codes.

\subsection{Thesis Contributions}

The main contributions of this thesis are:

We propose a fast trellis-based rate-allocation algorithm for robust transmission of progressively coded images over noisy channels. The algorithm, which is an improved version of a similar algorithm in [2], is based on the application of the Viterbi algorithm to a search trellis. This trellis is a substantially trimmed version of the one used in [2]. The proposed algorithm is applied to images encoded by SPIHT and JPEG2000 for transmission over noisy channels. For different total bit 
budgets and channel parameters, speed-up factors of up to about three orders of magnitude are achieved.

Rate-Compatible Low-Density Parity-Check (RC-LDPC) codes are proposed to provide unequal error protection for the robust and efficient transmission of progressively coded images over noisy channels. The total bit budget is partitioned between the source and channel coders, and optimal rates are assigned to source encoder packets. The performance of the proposed scheme is evaluated on binary symmetric channels (BSC). Experimental results indicate that the proposed scheme compares favorably with other joint source and channel coding schemes over a variety of channel conditions and transmission bit rates. In particular, the proposed scheme outperforms similar schemes based on turbo codes [2] and irregular repeataccumulate codes [3] by up to about $1.1 \mathrm{~dB}$ and $1 \mathrm{~dB}$ in the expected peak signalto-noise ratio (PSNR) of reconstructed images, respectively.

The proposed joint source and channel coding scheme is examined for uncorrelated/correlated Rayleigh flat-fading channels with/without side information (SI) using forward error correction (FEC). Simulation results show that our joint JPEG2000/RC-LPDC coding scheme can achieve the PSNR close to PSNR at capacity (an upper bound of PSNR for such channels). The smallest gap to the capacity is $0.7 \mathrm{~dB}$ (in PSNR). Furthermore, the application of RC-LDPC codes on to progressively coded images is more robust to estimation-error of channel parameters during transmission. Experiments shown that the when estimation-error of channel parameters happens to joint SPIHT/RC-LDPC coding system, our schemes outperform [5] and [6] by up to about $1.7 \mathrm{~dB}$ and $3 \mathrm{~dB}$ respectively. 
We also employ the proposed joint source and channel coding scheme for type-II hybrid ARQ communication channels. RC-LDPC codes are found to provide nearcapacity throughput for the channel. Thus, the amount of the correct recovered bitstream is maximized. Accordingly, we can allocate more bits to the source encoder so that the image can be reconstructed at a better quality (better PSNR). In the ARQ system, the images are transmitted over BSC and Rayleigh fading channels. Simulation results show that with type-II hybrid ARQ communication scheme, the PSNRs of the recovered images can be further improved over FEC scheme. The gaps to the capacity values are generally small, achieving the impressive performance for JSCC of images over noisy channels.

\subsection{Thesis Outline}

In Chapter 2, first we introduce the progressive source coding algorithms, including JPEG2000 and SPIHT, and channel codes - LDPC codes. Then, we explain the construction, encoding, and decoding of RC-LDPC codes, the channel codes used in our work.

In Chapter 3, we present the trellis construction for the rate-allocation problem and the VA of [2]. This is followed by a detailed explanation of the steps for simplifying the search trellis. Lastly, we present the simulation results for the simplified search algorithm and show that great speedups are possible with the proposed scheme over that of [2].

In chapter 4, we use RC-LDPC codes to protect progressively compressed images for transmission over BSC. An example is shown for transmission of JPEG2000 coded images. The overall structure of the proposed scheme is explained. Simulation results achieved with the proposed scheme are presented and compared with those of Turbo 
codes and IRA codes. Simulation results in the presence of channel estimation errors are also presented and discussed in this chapter.

Chapter 5 is organized in the following way: first, we give the reader a background on JSCC over fading channels. The Rayleigh fading channel model is presented with the corresponding configurations of RC-LDPC codes. The influence of estimation error of channel parameters on the performance is analyzed considering under-estimation and over-estimation. End-to-end PSNR figures are provided for transmitting JPEG2000 and SPIHT encoded images. The robustness of the proposed scheme is analyzed based on the results of error estimation of channel parameters for different Rayleigh fading channels.

In Chapter 6, type-II hybrid ARQ Scheme is briefly explained along with a throughput analysis of RC-LDPC codes. Then, we provide the structure of type-II hybrid ARQ Scheme. In order to compare to the FEC schemes, the end-to-end PSNR figures of the ARQ schemes are analyzes over both BSC and fading channels.

Finally, this thesis concludes with Chapter 7 , where we summarize our findings for the proposed JSCC using RC-LDPC codes over noisy channels. Based on the analysis of the results given in the previous chapters, we also address some directions that can be taken for future research. 


\section{Chapter 2}

\section{Joint Source and Channel Coding}

\subsection{Source Coding}

Image compression, a source coding technique, is used to remove redundancy of the image so that it can be transmitted with smaller number of bits on a band-limited channel. Channel coding, on the contrary, adds redundancy to achieve robustness in a noisy transmission environment. Source and channel coding are usually addressed as independent problems, but a better solution is available by solving the two problem jointly. To transmit images over noisy channels, the total bit budget has to be efficiently divided between source coding and channel coding so that a good end-to-end performance can be achieved.

In image processing domain, the quality of the received image can be quantitatively represented by PSNR, which is defined as:

$$
\operatorname{PSNR}(d B)=20 \log \frac{2^{B}-1}{R M S E}
$$

where $B$ is the number of bits to represent each pixel, RMSE is the root mean squared error between two images and it can be defined as: 


$$
R M S E=\sqrt{\frac{1}{N_{1} N_{2}} \sum_{n_{1}=0}^{N_{1}-1} \sum_{n_{2}=0}^{N_{2}-1}\left(x\left[n_{1}, n_{2}\right]-\hat{x}\left[n_{1}, n_{2}\right]\right)^{2}}
$$

where $N_{1}$ and $N_{2}$ are the size of the image, and $x\left[n_{1}, n_{2}\right]$ and $\hat{x}\left[n_{1}, n_{2}\right]$ are pixel values of the two images respectively.

An important feature of the state of the art image compression algorithms is embedded coding. By truncating the higher rate bit stream, we can easily obtain the lower quality description of the image. A natural application of embedded bit stream is progressive image transmission where the image is transmitted over a low bandwidth channel, and the receiver can improve the quality of the reconstructed image gradually when more bits are received. That means even if only a fraction of the bitstream is received, the image still can be reconstructed.

In the heart of current state-of-the-art image coders is the wavelet transform. Wavelet decompositions naturally represent image data in a hierarchical manner. Consequently, wavelet decompositions are suitable for progressive image compression. The resulting wavelet coefficients are grouped into approximation and detail coefficient sub-bands. Approximation coefficient sub-bands, calculated at each level of the filter bank structure, provide a low resolution approximation of the original image being represented. As the number of levels increases, this sub-band becomes increasingly coarse. The detail coefficient sub-bands provide details corresponding to the difference in information between the approximations as adjacent levels. As the number of levels increases, these details become increasingly coarse. Each detail sub-band contains wavelet coefficients that represent high-frequency components prominent in horizontal, vertical and diagonal spatial orietentations. This means that lower-frequency, less-detailed information is contained in 
the first transform level, while more-detailed, higher-frequency information is contained in further transform levels. As an example, the wavelet transform of the standard "Lena" image is shown in Figure 2.1. For simplicity, only two levels of the transform are shown here. The first transform level results in sub-bands LH1, HH1, HL1, and LL1. Only subband LL1 is passed on for further wavelet decomposition, generating the next transform level and creating sub-bands LH2, HH2, HL2, and LL2.
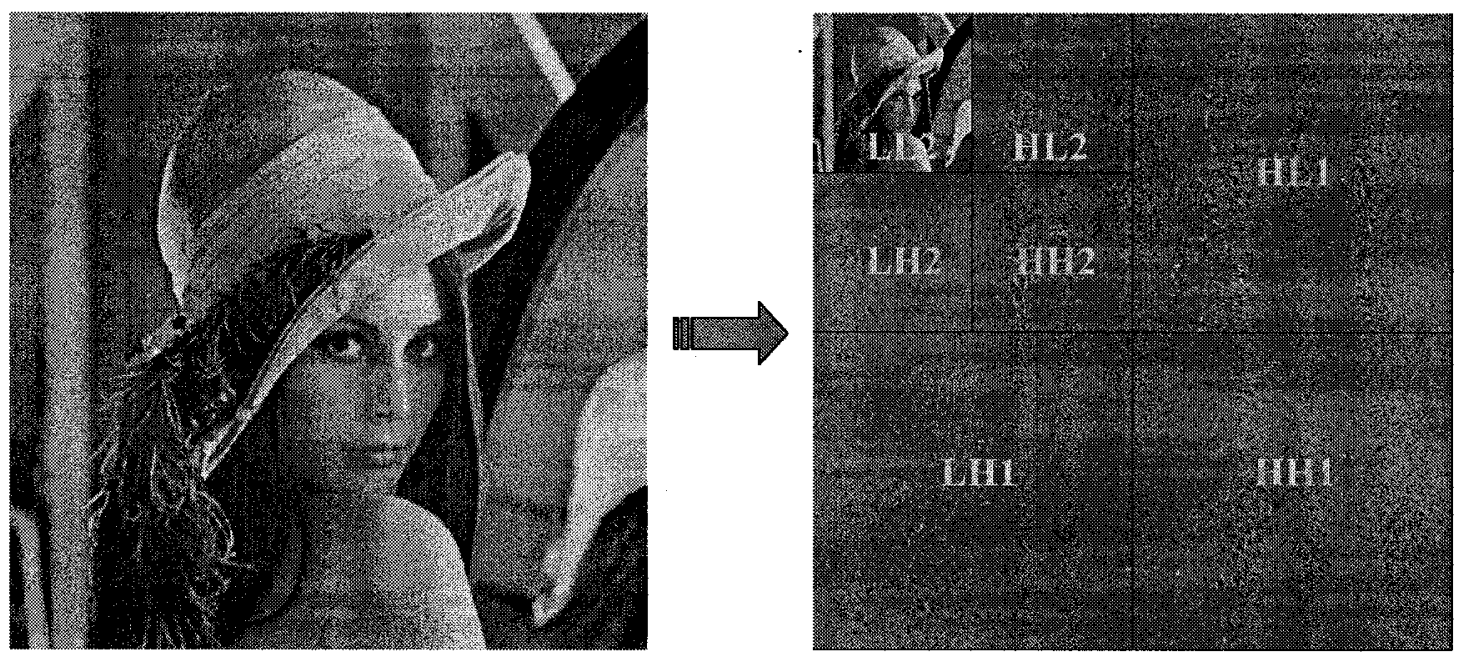

Fig 2.1: Two-level wavelet transform of the standard "Lena" image into sub-bands

Normally, the coefficients of the lowest frequency bands concentrate almost all of the wavelet transformed images energy and the high frequency bands of different scales and orientations show a strong self-similarity. These properties of DWT images can be exploited for efficient coding.

\subsubsection{SPIHT}

A coding algorithm developed for Discrete wavelet transform (DWT) transformed im- 
ages is the set partitioning in hierarchical trees algorithm (SPIHT) which has been developed by Said and Pearlman in 1996 [4]. DWT followed by embedded zero-tree (EZW) encoding is an efficient technique for image compression. DWT of an image results in a multi-scale representation of the image. The input image is passed through a filter bank structure using the wavelet transform.

The basic SPIHT algorithm, as it has been presented by Said and Pearlman makes intensive use of dynamic data structures to exploit the self similarities. The parent-child relations of the wavelet coefficients are shown in Figure 2.2.

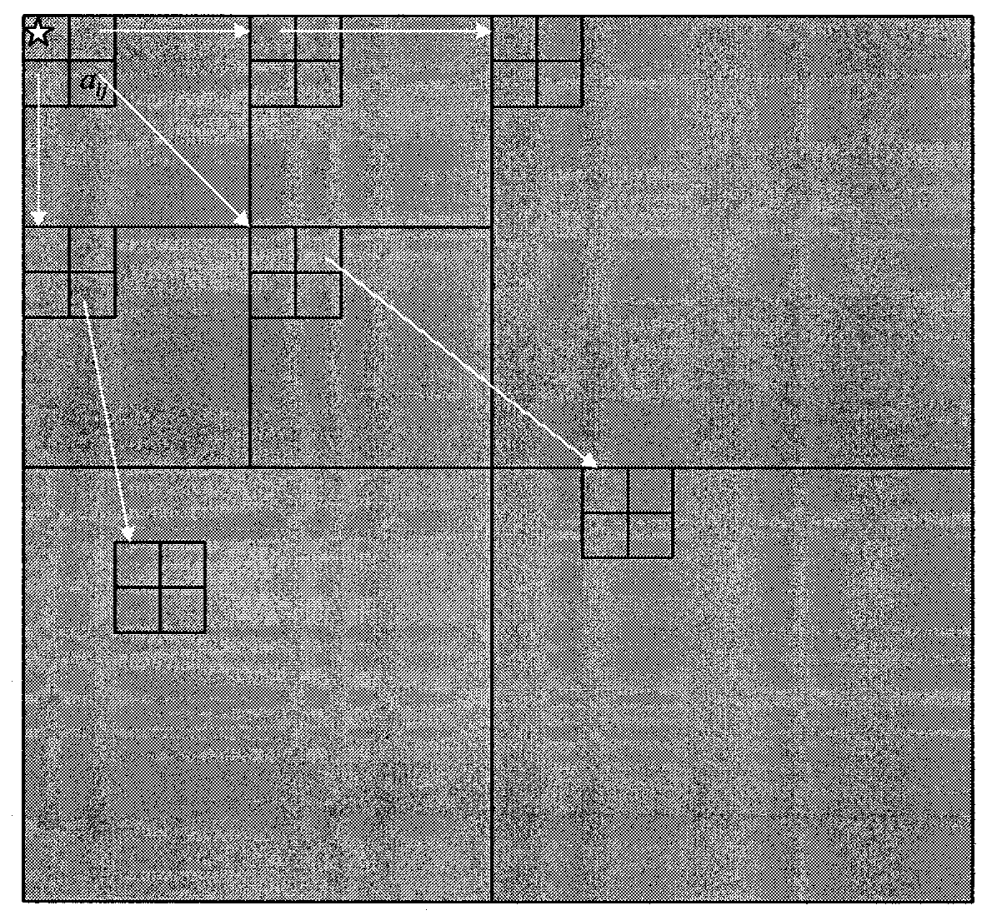

Figure 2.2: Illustration of the parent-child relation for the coefficients of SPIHT.

In order to exploit the self-similarities during the coding process, oriented trees of four offspring are taken for the representation of a wavelet transformed image. Each node of the trees represents a coefficient of the transformed image. The levels of the trees consist of coefficients at the same scale. The trees are rooted at the highest scale of the represen- 
tation. The SPIHT algorithm assumes that each coefficient $a_{i j}$ is a good predictor of the coefficients which are represented by the sub-tree rooted by $a_{i j}$. The overall procedure is controlled by an attribute, which gives information on the significance of the coefficients. More formally, a coefficient is insignificant with respect to a threshold $t$ if its magnitude is smaller than $2^{t}$. Otherwise it is called significant with respect to the threshold $t$.

In the basic SPIHT algorithm, the coefficients of a wavelet transformed image are classified into three sets, namely the list of insignificant pixels (LIP ) which contains the coordinates of those coefficients that are insignificant with respect to the current threshold $t$, the list of significant pixels (LSP) which contains the coordinates of those coefficients which are significant with respect to $t$, and the list of insignificant sets (LIS) which contains the coordinates of the roots of insignificant sub-trees. During the compression procedure, the sets of coefficients in LIS are refined and if coefficients become significant they are moved from LIP to LSP. The bitstream can thus be progressively organized.

\subsubsection{JPEG2000}

The current image compression standard JPEG2000 [7], [8] employs an embedded coding scheme using the state-of-the art compression techniques based on wavelet transform with optimal truncation (EBCOT), an efficient algorithm to construct the bitstream.

In JPEG2000, equally sized code blocks, which are essentially bit streams of data, are generated within each sub-band. This break-down is necessary for coefficient modeling and coding, and is done on a code-block-by-code-block basis. In essence, the actual compression is achieved by truncating and/or re-quantizing the bit streams contained in each code block. These bit streams are then optimally truncated using a technique known as 
post-compression-rate-control (PCRC).

Code blocks can be accessed independently. Their bit streams are coded with three coding passes per bit plane. This process, called context modeling, is used to assign information about the importance of each individual coefficient bit. The code blocks can then be grouped according to their significance. On the decoding side it is then possible to extract information according to its significance, allowing the most significant information to be decoded first.

Tiles or images are further partitioned into precincts. Precincts contain a number of code blocks, and are used to facilitate access to a specific area within an image in order to process this area in a different way, or to decode only a specific area of an image. The JPEG2000 bitstream is generated by arranging code blocks or precincts into an array of packets with the lower sub-bands coming first. The JPEG2000 stream starts with a main header containing information such as: uncompressed image size, tile size, number of components, bit depth of components, coding style, transform levels, progression order, number of layers, code block size, wavelet filter type, quantization level, etc. The entire image data, grouped in code blocks of LL, HL, LH, and HH sub-bands, follows the header. Data is not contained in the header information. Also, a table of contents can be stored on the encoder side, and allows a decoder to call up a certain resolution on demand, without first having to decode or download the entire JPEG2000 code stream.

In our work, the original images are first encoded by JPEG2000 or SPIHT encoder. In order to make our system standard compatible, we make no change to the organization of the source coded bitstream. To obtain the distortion of each packet required as the input 
for rate allocation algorithm, we just trace the organization of the bitstream, so the data in the packet can be mapped into the coefficients in the transform domain. Here we do not have to decode the bitstream. From the transform domain, we can easily calculate the distortion values by finding the mean squared error (MSE) of the truncated bitstream translated back to the coefficients in the transform domain. For JPEG2000 algorithm, each code block provides the distortion value at any truncation point within the code block. Taking advantage of this information, we can obtain the distortion of the packet by adding up the distortion values for all the previous code blocks and the correctly received bits of the current code block.

\subsection{Channel Codes}

\subsubsection{LDPC codes}

LDPC codes were invented by Robert Gallager in his $\mathrm{PhD}$ thesis [9] and rediscovered several times for the next 30 years [10],[11].

LDPC codes are linear block codes obtained from sparse bipartite graphs (called Tanner graphs). Suppose that $G$ is a graph with $n$ left nodes (called message or bit nodes) and $r$ right nodes (called check nodes). In this work we are focused on binary codes. The graph gives rise to a linear code of block length $n$ and dimension at least $n-r$ in the following way: The $n$ coordinates of the codewords are associated with the $n$ message nodes. The codewords are those vectors $\left(c_{1}, \ldots, c_{n}\right)$ such that for all check nodes the sum of the neighboring positions among the message nodes is zero. Figure 2.3 illustrates an example of the Tanner graph. 


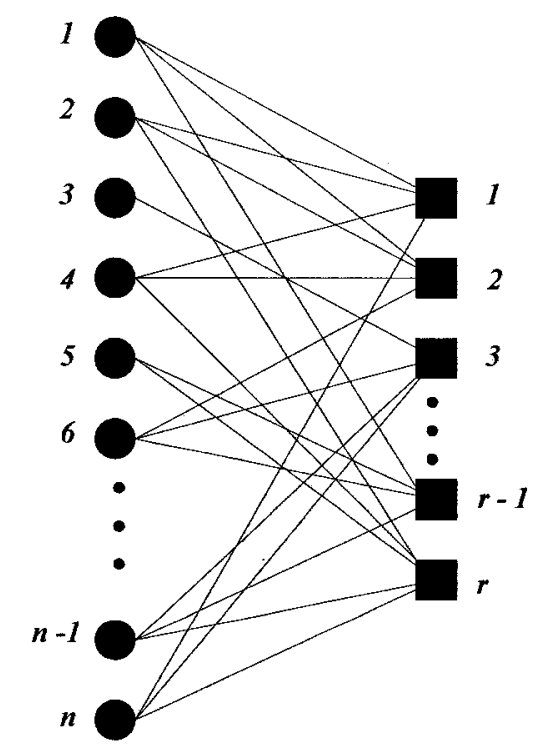

Figure 2.3: Tanner graph of LDPC codes.

The graph representation is analogous to a matrix representation by looking at the adjacency matrix of the graph: let $H$ be a binary $r \times n$ matrix in which the entry $(i ; j)$ is 1 if and only if the $i$ th check node is connected to the $j$ th message node in the graph. Then the LDPC code defined by the graph is the set of vectors $c=\left(c_{1}, \ldots, c_{\mathrm{n}}\right)$ such that $H \cdot c^{\mathrm{T}}=0$. The matrix $H$ is called a parity check matrix for the code. Conversely, any binary $r x_{n}$ matrix gives rise to a bipartite graph between $n$ message and $r$ check nodes, and the code defined as the null space of $H$ is precisely the code associated to this graph. Therefore, any linear code has a representation as a code associated to a bipartite graph (note that this graph is not uniquely defined by the code). However, not every binary linear code has a representation by a sparse bipartite graph. If it does, then the code is called a lowdensity parity-check (LDPC) code. The sparsity of the graph structure is the key property that allows for efficient decoding of LDPC codes using iterative decoding algorithm [10] and [12].

A Tanner graph of LDPC codes is called regular if every message node is connected to 
equal number of check nodes and every check node is connected to equal number of message nodes. Otherwise, the LDPC codes are called irregular. An ensemble of LDPC codes is determined by degree distribution pairs. The degree distribution of message nodes is defined as:

$$
\lambda(x)=\sum_{i \geq 1}^{d_{s}^{\max }} \lambda_{i} x^{i}, \text { where } \lambda_{i} \text { is the fraction of message nodes with degree } i, d_{s}^{\max } \text { is the }
$$
largest entry of all the message nodes. Likewise, the degree distribution of check nodes is defined as:

$$
\rho(x)=\sum_{i \geq 2}^{d_{c}^{\max }} \rho_{i} x^{i}, \text { where } \rho_{i} \text { is the fraction of check nodes with degree } i, d_{c}^{\max } \text { is the larg- }
$$

est entry of all the check nodes.

Similar to turbo codes, LDPC codes with iterative decoding provide very good performance over a variety of channels with reasonably low complexity. (For more information on LDPC codes and iterative decoding, the reader is referred to [12] and the references therein.) In particular, irregular LDPC codes [13] are known to outperform regular codes and turbo codes and to approach the capacity of several channels at large block lengths [13]. Moreover, compared with turbo Codes, LDPC codes exhibit several other advantages [12], [13]: i) Iterative decoding algorithms for LDPC codes are parallelizable and can be realized at much faster speed than turbo decoders. ii) Almost all the errors are detectable. The only drawback of LDPC codes is its encoding complexity. The encoding complexity of LDPC codes is in general quadratic in $n$. 


\subsubsection{RC-LDPC codes}

RC-LDPC codes [1] are an embedded sequence of LDPC codes. They inherit the advantages of LDPC codes and provide extra advantages. A single encoder and decoder can be used for them. We use a particular construction for RC-LDPC codes that can accommodate irregular degree distributions, linear-time encodability. RC-LDPC codes are based on progressive-edge-growth (PEG) [14] construction which is one of the best known constructions for short LDPC codes.

Our RC-LDPC codes are irregular and are constructed by PEG algorithm based on the construction of [1]. Similar to turbo codes and IRA codes, our codes are linear-time encodable, because of the lower-triangular format of the $H$ matrix. Moreover, because of rate-compatibility, they can all be easily decoded using the decoder for the lowest rate code and by substituting the missing parity bits with erasures [1]. PEG construction is known to generate very good irregular LDPC codes at finite block lengths if used with symbol-node degree distributions optimized by density evolution [12], [13].

In the context of progressive coding, the source encoder's bit stream has a hierarchical organization such that bits are ordered in terms of their importance. A better result can then be obtained using unequal error protection (UEP) [2], [3], [15]-[20]. An attractive framework for application of UEP is to use rate-compatible (RC) codes, where the parity bits for higher rate codes are embedded in those of lower rate codes. One of the main advantages of using RC schemes is that all the codes in the sequence can be encoded and decoded using a single encoder/decoder pair. In [15]-[20], RC punctured convolutional codes (RCPC) are used for transmission of progressively coded images, while in [20], [2] and [3], Reed-Solomon codes, RC turbo codes (RCTC) and irregular repeat-accumulate 
(IRA) codes are employed, respectively.

\subsubsection{PEG Construction}

The mother code matrix and the extension matrix are constructed with linear-time PEG construction [1]. We know that a cycle is a closed path that begins with a node and ends with itself. The girth of a graph is defined as the number of edges of the shortest cycle in the graph. An efficient method for constructing Tanner graphs with a large average girth, in a greedy sense, is PEG construction. It is important to have graphs with good girth distributions as iterative decoding algorithms perform better on such graphs [21]. The edges between the message nodes and the check nodes are progressively established between bit nodes and check nodes of the Tanner graph in an edge-by-edge manner.

In fact, constructing a Tanner graph with the largest possible girth is a rather difficult combinatorial problem, and thus we have to look for sub-optimal algorithms that are feasible in practice and construct a Tanner graph with a relatively large girth. The PEG algorithm is one such algorithm that maximizes the local girth of a symbol node whenever a new edge is added to this symbol node.

\subsubsection{Encoding and Decoding of LDPC}

\section{Codes}

One of the drawbacks of LDPC codes is the encoding complexity. The problem is overcome in our RC-LDPC codes. The parity check matrix of our LDPC codes is organized in 
lower-triangular format so that the LDPC codes are linear-time encodable. From the parity check matrix of our RC-LDPC codes, we know that it is just in the shape of lowertriangular format. This is another advantage of our RC-LDPC codes: the simple encoding process.

First, we start by describing a general class of decoding algorithms for LDPC codes. These algorithms are called message passing algorithms, and are iterative algorithms. The reason for their name is that at each round of the algorithm, messages are passed from message nodes to check nodes, and from check nodes back to message nodes. The messages from message nodes to check nodes are computed based on the observed value of the message node and the messages passed from the neighboring check nodes to that message node. An important aspect is that the message that is sent from a message node $v$ to a check node $c$ must not take into account the message sent in the previous round from $c$ to $v$. The same is true for messages passed from check nodes to message nodes. One important message passing algorithms is the belief propagation (BP) algorithm [10]. This algorithm was first presented by Gallager [9]. The messages passed along the edges in this algorithm are probabilities, or beliefs. More precisely, the message passed from a message node $v$ to a check node $c$ is the probability that $v$ has a certain value given the observed value of that message node, and all the values communicated to $v$ in the prior round from check nodes incident to $v$ other than $c$. On the other hand, the message passed from $c$ to $v$ has the probability that $v$ has a certain value given all the messages passed to $c$ in the previous round from message nodes other than $v$. It is easy to derive formulas for these probabilities under a certain assumption called independence assumption. It is advantageous in the sense of computation complexity to work with likelihoods, or some- 
times even log-likelihoods instead of probabilities. The iterative decoding procedure stops when the codeword satisfies the check constraints or the maximum number of iteration is reached.

\subsubsection{General Construction of RC-LDPC Codes}

Different rates for RC-LDPC codes [1] are obtained by puncturing and extending a mother code whose rate is carefully selected.

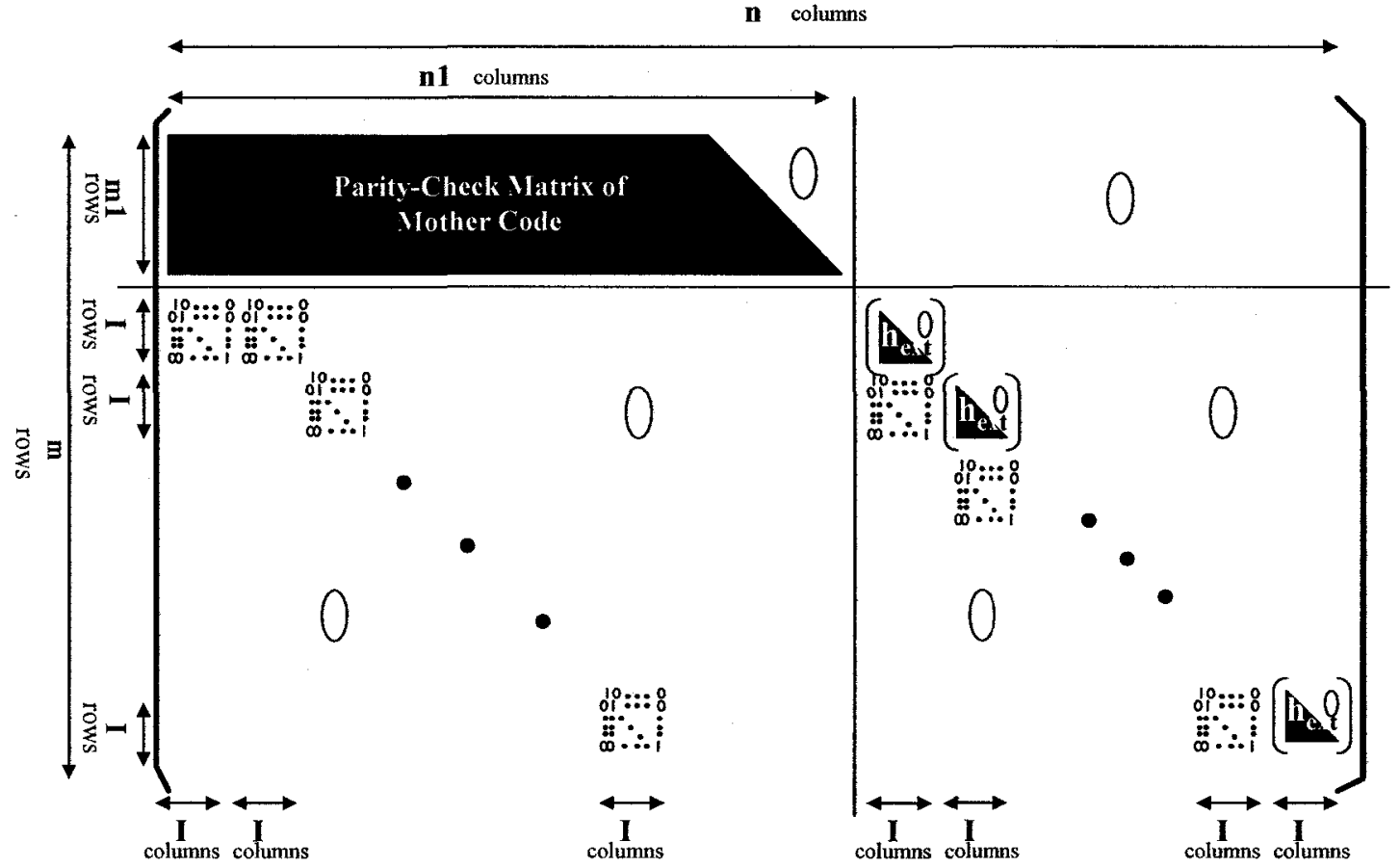

Figure 2.4: General Construction of the parity-check matrices for the mother code and extended codes in the sequence of RC-LDPC codes 
We first construct a mother code and then generate the lower rates by extending the parity-check matrix of the mother code and the higher rates by puncturing. The rate selection for the mother code and different levels of puncturing and extending should be performed carefully. To explain the construction of RC-LDPC codes, let us look at the parity-check matrix $H(m \times n)$ of the code in Figure 2.4. From Figure 2.4, we know that $H$ is composed of mother code matrix $\left(m_{1} \times n_{1}\right)$, extension matrix $h_{\mathrm{ext}}(I \times I)$ and identity matrix $(I \times I)$. The mother code matrix provides the basic rates of the code. Based on the rate of mother code, puncturing and extension can be applied to the mother code matrix to generate the desired set of channel coding rates.

The advantage of RC-LDPC codes comes from the embedded structure of the codes. Only one decoder is necessary. To decode the codeword with different rates, we simply substitute the puncturing and/or extension bits for erasure and decode it with the decoder that corresponds to the lowest rate. The embedded structure could also maximize the throughput for a type-II hybrid ARQ scheme. RC-LDPC codes in [1] are optimized and the throughput is maximized based on AWGN channel. In our work, we optimize RCLDPC codes for BSC and Rayleigh fading channels and apply them on to progressively coded images. 


\section{Chapter 3}

\section{Rate-Allocation Algorithm}

\subsection{Background}

Equal error protection (EEP) allocates code protection to all the parts of the bitstream regardless of the contribution of each part to the quality of the image. The bitstream of progressively coded image, such as those generated SPIHT and JPEG2000, is organized such that the contribution of each part is different. So unequal error protection (UEP) is desirable for robust transmission of progressively coded images over noisy channels [2], [15], [22] and [23]. The problem of rate allocation, which is in the heart of such joint source-channel coding scenarios can, in principle, be broken into two sub problems: i) optimal division of the total available bit budget for the transmission of image between source and channel coding; and ii) optimal assignment of the channel coding share of the bit budget among source packets. The two optimizations can be performed jointly with a variety of cost functions. Two well-known cost functions are average mean squared error (MSE) distortion (or equivalently, average PSNR), and the average number of correctly received source bits [22], [23]. Following [22], we refer to the corresponding optimal solutions as distortion-based optimal and rate-based optimal (or briefly, distortion optimal 
and rate optimal). In [23], Chande and Farvardin proposed a dynamic programming approach for finding the distortion and rate optimal solutions. Their algorithm has a complexity of $O\left(N^{2}\right)$ and $O(N)$ for the two cases, respectively, where $N$ is the number of transmitted packets. While the rate optimal solution has a lower complexity and enjoys other advantages such as independence from the image and from the source coder performance, it can result in significant performance loss compared to the distortion optimal solution [22] (in an example of [22], up to 3dB degradation in PSNR for a JPEG2000 coded image is reported). Nosratinia et al. [15] developed a parametric approach to solve the rate allocation problem that produced a distortion optimal solution. Their approach which is based on creating an empirical model for bit error rate is generally less complex than non-parametric methods. Hamzaoui et al. [22] proposed a suboptimal approach for finding the distortion optimal solution to reduce the complexity of Chande et al.'s algorithm. Their approach, which starts from a rate optimal solution, performs a local search and converges to a locally distortion optimal solution. For the tested images, degradation in PSNR within only about $0.3 \mathrm{~dB}$ compared to the distortion optimal solution is reported. A variation of this approach has also been used in a very recent paper by Lan et al. [2]. Another suboptimal approach for finding the distortion optimal solution based on the Viterbi algorithm (VA) (forward dynamic programming) was presented in [1]. For the cases where code rates have a common numerator or denominator, ${ }^{1}$ the VA has also a complexity of $O\left(N^{2}\right)$. In fact, according to the results of [3], if the number of possible channel coding rates is small, the complexity of the VA of [2] is comparable with that of

\footnotetext{
${ }^{1}$ Note that these conditions should not be viewed as serious constraints on the channel code construction. In fact, for many instances of rate compatible codes, these conditions are natural consequences of the code construction (see, e.g., [1], [2]).
} 
the local search of [22]. It is only when the number of rates increases that the complexity of the latter tends to be lower than that of the former. The VA of [1] performs similarly to the local search of [22] (see [3], [22]).

In this work, we simplify the algorithm of [2] by significantly reducing the number of branches on the search trellis. While these simplifications have little to no effect on the performance of the rate-allocation algorithm, they reduce its complexity substantially (in our examples, by nearly three orders of magnitude).

\subsection{Improved Rate-Allocation Algorithm}

We simplify the search trellis in four ways. First, we observe that the optimal solution to subproblem (i), i.e., the optimal ratio of bit budgets for source and channel coding, is rather insensitive to the compressed image and the total bit budget, and is mainly a function of the channel parameter. This observation can significantly reduce the number of candidate final nodes on the trellis. Secondly, we show that there are branches on the trellis that will never have the chance of being on the survivor path for the VA. By trimming these branches, we further simplify the trellis. Thirdly, we also reduce the number of branches emanating from a node from $M$, the number of channel coding rates, to $M^{\prime}<M$. This is performed based on the error rate curves of the channel codes and that, for a given channel parameter, only a few of the codes can be useful in providing proper protection efficiently. Because in the full search the number of branches on the trellis increases quadratically with $M$, this modification will also considerably reduce the search complexity. Finally, more simplification is possible by limiting our search to rate allocations with non-decreasing rates. 
To explain our rate-allocation algorithm, we use similar assumptions as in [2]. We consider transmission of progressively coded images over a binary symmetric channel (BSC) with parameter $\varepsilon$, known to both transmitter and receiver. A set of channel codes with rates $r=\left\{r_{1}, \ldots, r_{M}\right\}$ is used to protect the source packets. We specify the available total bit budget, $B$. The rate-allocation algorithm at the transmitter is responsible for finding the distortion optimal solution by dividing $B$ between source and channel encoders and by assigning optimal rates to each source packet to minimize the average distortion. At the receiver, the packets are received and decoded and the image is reconstructed with increasing accuracy as more packets arrive. We assume that the system is able to detect errors and that we discard the first erroneous packet and all subsequent packets. Assuming that the number of transmitted packets is $N$, the rate-allocation algorithm assigns rates $R_{l}$, $\ldots, R_{N}$ to the packets, where $R_{i} \in r, \forall i$. The cost function (average distortion) is given by

$$
D=D_{0} P_{1}+\sum_{n=1}^{N-1} D_{n} P_{n+1} \prod_{i=1}^{n}\left(1-P_{i}\right)+D_{N} \prod_{i=1}^{N}\left(1-P_{i}\right)
$$

where $D_{n}$ is the distortion (mean squared error (MSE)) associated with the erroneous $(n+1)$ th packet (assuming all the previous packets have been received correctly), and $P_{i}$ is the error probability of the $i$ th packet, which is the word error rate (WER) of the channel code with rate $R_{i}$. The image encoder supplies values for $D_{n}$ while the WER curves of the channel codes yield values for $P_{i}$.

One may packetize the source output and construct the transmitted packets using two approaches. In the first, source packets have all the same length and thus the lengths of the packets after channel coding would be different [15], [23]. In the second approach, transmitted packets have all the same length $\left(L_{I}=\ldots=L_{N}=L\right)$ and thus depending on 
the channel coding rate, source packets would have different lengths [2], [3], [22]. Although the algorithm of [2] has been designed for the latter scenario, we notice that one may apply it equally effectively to the former. The only difference is that the final nodes of the search trellis will occur at different levels, as shown in Figure 3.1 (a).

Suppose that the channel codes have all the same information block length $k$, where $k$ is an integer multiple of an integer number $I$, i.e., $k=d \times I$. In this case, the source packets all have the same length $k-\delta$, where $\delta$ represents the overhead required for transmitting side information such as the rate of the next packet to the receiver and error detection if the error correcting codes do not have that capability. Assume also that the block lengths for all the channel codes are integer multiples of $I$, i.e., $n_{i}=l_{i} \times I$. The rates for channel codes are thus $r_{i}=k / n_{i}=d / /_{i}{ }^{2}$ Based on the approach in [1], to minimize (3.1) with the constraint $\sum_{i=1}^{N} L_{i}=B$, we construct a trellis such that each section of the trellis represents a transmitted packet. Branches represent different code rates $\left(R_{i}\right)$ and nodes (states) represent the number of coded bits. To each node at level $i$ of the trellis, we assign an integer number $S_{i}=\sum_{j=1}^{i} l_{m_{j}}$, where $m_{j} \in\{1, \ldots, M\}$, and refer to this number as the state index for the node. Clearly all the nodes at a given level of the trellis represent the same number of source bits. We terminate the construction of the trellis at a node $U$ if the total bit budget is fully consumed or if the remaining budget is not enough to send another packet $(<k)$. In this case, node $U$ is referred to as a final node of the trellis. Figure 3.1(a) shows a simple example of such a trellis for $r=\{2 / 2,2 / 4,2 / 5$,

\footnotetext{
${ }^{2}$ The case where the rates have a common denominator, instead of a common numerator, has been discussed in [2]. Although in this paper we consider rates with a common numerator, a similar treatment can also be applied to the other case.
} 
$2 / 6,2 / 7\}$. In this example, $d=2 ; l_{1}=2, l_{2}=4, l_{3}=5, l_{4}=6, l_{5}=7$; and we assume that the total bit budget is $9 k$. There are two final nodes with indices 17 and 18 at each level of the trellis starting from level 3 and beyond.

After the construction of the trellis, the VA is applied to the trellis. The forward pass of the VA consists of computing the average distortion of (3.1) for each incoming branch to a node and then selecting the branch with the minimum distortion. After the whole trellis is processed one level at a time, the algorithm selects the final node with the minimum distortion. The trellis level at which this node is located equals the optimal number of packets $\left(N^{*}\right)$, from which the optimal allocated number of source bits $\left(N^{*}(k-\delta)\right)$ and the source compression ratio can be determined. We find the optimal channel coding rates by tracing back the survivor path for this particular node.

The complexity of the VA is proportional to the number of trellis branches. In the following, we show that the number of trellis branches can be significantly reduced at no cost in performance. We explain the trimming process of the trellis in four steps. In general, in the trimming process, when a node is eliminated, all the incoming branches will be trimmed and when all the outgoing branches of a node are trimmed, then the node will be eliminated. 


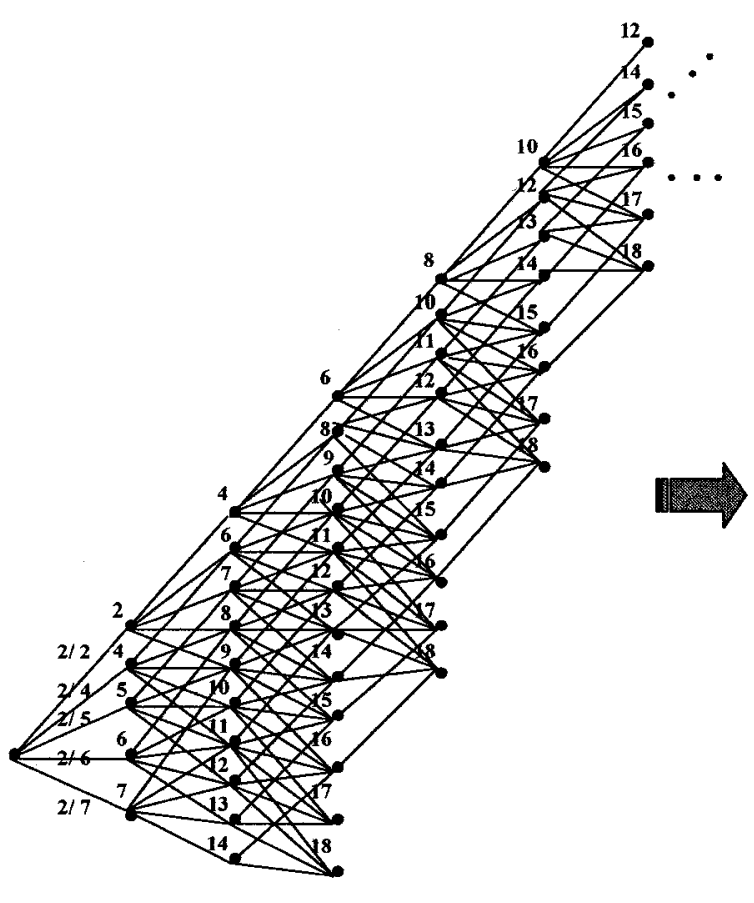

(a)

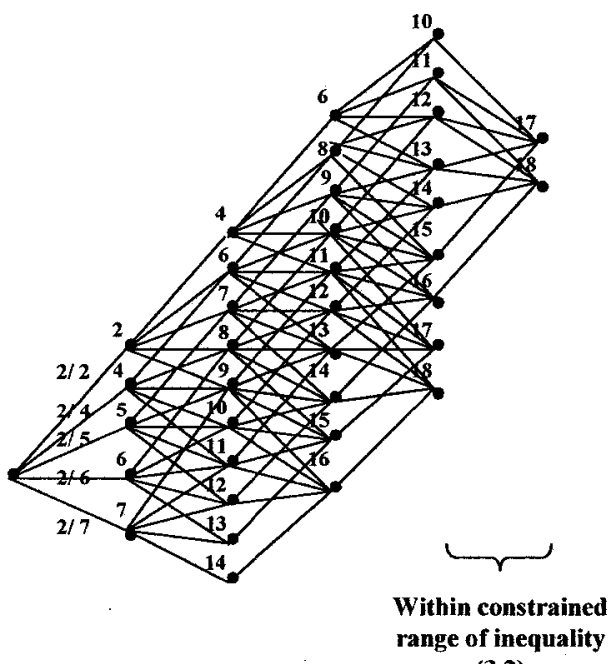

(3.2)

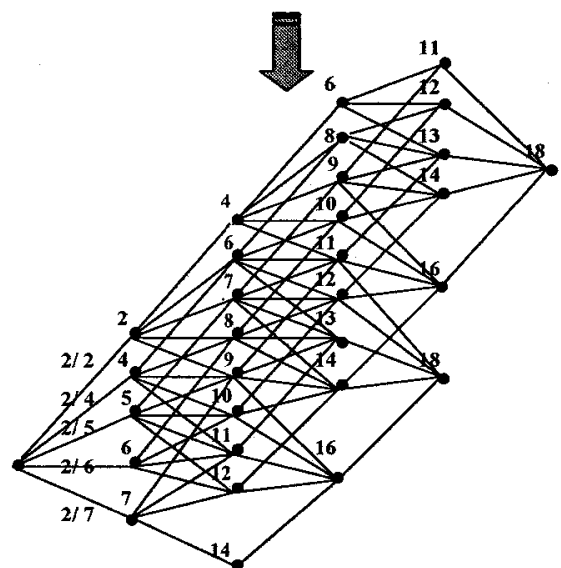

(c)

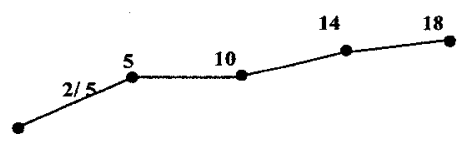

(e)

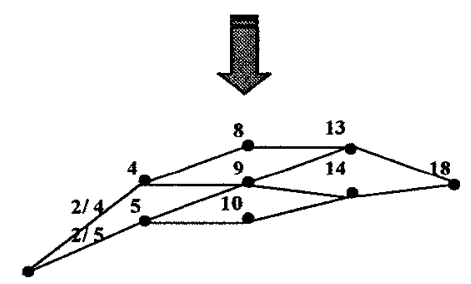

(d)

Figure 3.1: Illustration of the process of trimming the trellis 
First, we demonstrate that only the candidate (final) nodes in a small number of trellis levels need to be tested to obtain the one with the minimum distortion. Suppose that for a particular instance of the rate-allocation problem we know the optimal ratio $(\alpha)$ of the number of source bits $(k N)$ to the total bit budget $(B)$. Assuming that $\alpha B$ is divisible by $k$, we can easily calculate the optimal number of packets by $N^{*}=\alpha B / k$, thereby limiting the search to only the candidate nodes at a single level of the trellis. In the following section we obtain an empirical estimate for $\alpha$ which is only a function of the channel parameter and is independent of the image and the total bit budget. Knowing the channel parameter $\varepsilon$, we can thus easily obtain this estimate, $\hat{\alpha}(\varepsilon)$ (which will be given later), and then use the following inequality

$$
\frac{\left(\hat{\alpha}(\varepsilon)-\eta_{l}\right) B}{k}<N^{*}<\frac{\left(\hat{\alpha}(\varepsilon)+\eta_{u}\right) B}{k}
$$

to limit the search for the candidate nodes. In inequality (3.2), parameters $\eta_{l}$ and $\eta_{u}$ represent the estimation error, and are determined empirically. We subsequently show that only few trellis levels (in our examples at most 3) satisfy inequality (3.2) and need to be checked. Figure 3.1 (b) shows how to trim the search trellis of Figure 3.1 (a) using this constraint.

The next step in trimming the trellis involves removing some of the branches that have no chance of being part of the survivor path. For this, we use the following lemma.

Lemma 1: Consider two paths on the trellis starting from a common node $U$ and ending at final nodes $V_{i I}$ and $V_{i 2}$, both at level $i$ of the trellis. If the rates for all the branches of the path between $U$ and $V_{i 1}$ are less than or equal to the corresponding rates of the path between $U$ and $V_{i 2}$, then the latter path cannot be part of the final survivor path. 
The proof of Lemma 1 follows from the observation that the source packets on the path between $U$ and $V_{i I}$ are on average better protected and thus result in lower average distortion compared with the path between $U$ and $V_{i 2}$. If the situation described in Lemma 1 occurs, we say that the path between $U$ and $V_{i l}$ dominates the path between $U$ and $V_{i 2}$. The following proposition enables one to trim some incoming branches to the final nodes.

Proposition 1: For an incoming branch $e$ to a final node on the trellis, if all the paths which include $e$ are dominated by other paths on the trellis then $e$ can not be part of the survivor path and may be trimmed.

Proposition 1 in fact applies to any branch of the trellis. However while testing the condition of Proposition 1 for incoming branches to final nodes is rather simple (as we will see in the following), for intermediate branches it can be a tedious task.

Consider a trellis construction similar to Figure 3.1 (a), where the channel coding rates for the outgoing branches from each node are ordered from the top to the bottom according to their denominators. In such a construction, the state indices at each level of the trellis are also similarly ordered (i.e., if $S_{i j}<S_{i k}$ at level $i$ of the trellis, then the node with in$\operatorname{dex} S_{i j}$ is located at the top of the one with index $S_{i k}$ ). Let $\Omega$ denote the set of trellis levels that satisfy (3.2). For every $i \in \Omega$, we consider all the incoming branches to the final nodes at level $i$. For each such branch $e=\left(U^{\prime}, U\right)$, if there exists another branch $e^{\prime}$ with the same initial node $U^{\prime}$ but with the end node $V$ having a larger index than that of $U$ ( $V$ is positioned below $U$ in the trellis), then one may trim $e$ according to Proposition 1. If a branch with the property of $e^{\prime}$ can not be found, we extend the search to level $i-2$ by testing the set of all the two-branch paths $\psi_{2}$, with each path consisting of $e^{\prime \prime}$ and $e$, where

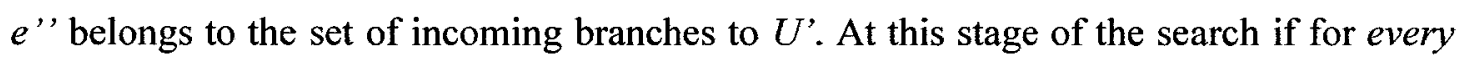


path $\pi$ in $\psi_{2}$ we can find a two-branch path that dominates $\pi$ (graphically, this path must be positioned on or below $\pi$ on the trellis), then one may use Proposition 1 to trim $e$. If a dominating path does not exist for any of the paths in $\psi_{2}$ then the search should extend to level $i-3$ for that path. This continues until we reach the root of the trellis. If by then we are able to find dominating paths for all the paths that include $e$, then $e$ can be trimmed according to Proposition 1. Let $S_{i}=\left\{S_{i l}, \ldots, S_{i J i}\right\}$ denote the set of the indices for the final nodes at level $i$, with $S_{i l}>S_{i j}, \forall S_{i j} \in S_{i} \backslash S_{i 1}$. At each level $i$, the trimming process tends to eliminate more branches from final nodes with lower indices. In many cases, all the branches of some of these nodes and thus the nodes themselves are trimmed, and quite often, just the node with index $S_{i l}$ will survive.

Figure 3.1 (c) illustrates the application of this process to further simplify the search trellis in Figure 3.1 (b). Two final nodes exist at each of levels 4 and 5 of the trellis in Figure 3.1 (b). Note that five incoming branches lead to the node with index 17 at level 4. Of these five branches the three middle ones are trimmed at level 3. In order to trim the other two, one needs to trace their paths to the root of the trellis. After all its incoming branches have been trimmed node 17 at level 4 will be removed. In addition, after all the outgoing branches of node 15 at level 3 are trimmed, node 15 itself and its incoming branches will be eliminated. This in turn leads to the removal of node 13 at level 2 and all its incoming branches.

In our example of next section, by applying Proposition 1, all but one final node at each level of the trellis is always trimmed (similar to Figure 3.1 (c)).

In addition to the foregoing two simplifications, we may further reduce the complexity of the search trellis by noticing that for a given channel parameter only a small number 
$M^{\prime}$ of the $M$ available channel codes are useful in an optimal rate-allocation scheme. These codes have adjacent rates. The highest rate is the largest of those having a WER less than one while the lowest rate is the largest of those having a WER below a certain small positive value $P^{*}$. Based on our simulation results, choosing $P^{*}=10^{-4}$ results in no degradation in performance as WERs below $10^{-4}$ are effectively equivalent to zero error probability in the calculation of PSNR. Figure 3.1 (d) demonstrates the application of this constraint to further trim the trellis of Figure 3.1 (c), assuming that only the channel codes with rates $2 / 4$ and $2 / 5$ have been used.

Finally, assuming that the optimal channel coding rates for the transmission of successive source packets are non-decreasing, ${ }^{3}$ we can further simplify the trellis by trimming all the emanating branches of every node that have rates smaller than the minimum rate of the incoming branches to the node. Figure 3.1 (e) displays the result of applying this process to Figure 3.1 (d).

It should be noted that all the simplifications to the search trellis explained in this section are applied only once and off-line. The four steps of our rate-allocation algorithm can be implemented at the same time and the order of them can be switched. The simplified trellis will then be used for rate-allocation using the VA.

\footnotetext{
${ }^{3}$ [15] proves that under certain assumptions the optimal rates are in fact non-decreasing. Also [22] shows that the distortion optimal rate allocation with the constraint of non-decreasing rates, which the authors call the "constrained distortion optimal" solution, performs effectively the same as the unconstrained solution.
} 


\subsection{Performance of the Fast Trellis-Based}

\section{Rate-Allocation Algorithm}

In our simulations, we use a sequence of channel codes with rates $8 / 8,8 / 10,8 / 11,8 / 12$, $8 / 13,8 / 15,8 / 16,8 / 18,8 / 20$ and $8 / 22$, and with information block length of $k=3072=8 \times$ 384 bits. Figure 3.2 shows the WER curves for these codes over BSC. ${ }^{4}$ Similar to [2], we use the standard $512 \times 512$ 8-bit monochrome "Lena", "Goldhill" and "Barbara" images to carry out simulations having total bit budgets of 0.252 bit per pixel (bpp), $0.505 \mathrm{bpp}$ and $0.994 \mathrm{bpp}$. We use the standard JPEG2000 compression software and apply the same algorithm as in [2] to find the optimal rate-allocation for the three images under various transmission bit budgets and crossover probabilities of the BSC. Figure 3.3 shows the optimal ratio of the source to total bit budgets for the nine combinations of images and total bit budgets.

\footnotetext{
${ }^{4}$ These codes are in fact rate-compatible low-density parity-check (LDPC) codes optimized for BSC [14]. Under the same conditions these codes outperform similar schemes based on turbo codes [2] and irregular repeat-accumulate codes [3] by up to $1.1 \mathrm{~dB}$ and $1 \mathrm{~dB}$ in PSNR for transmission of JPEG2000 images, respectively [24]. These improvements in performance, however, are irrelevant to our work in this chapter.

${ }^{5} I$ will explain the construction of these codes in more detail in Section 4.2.
} 


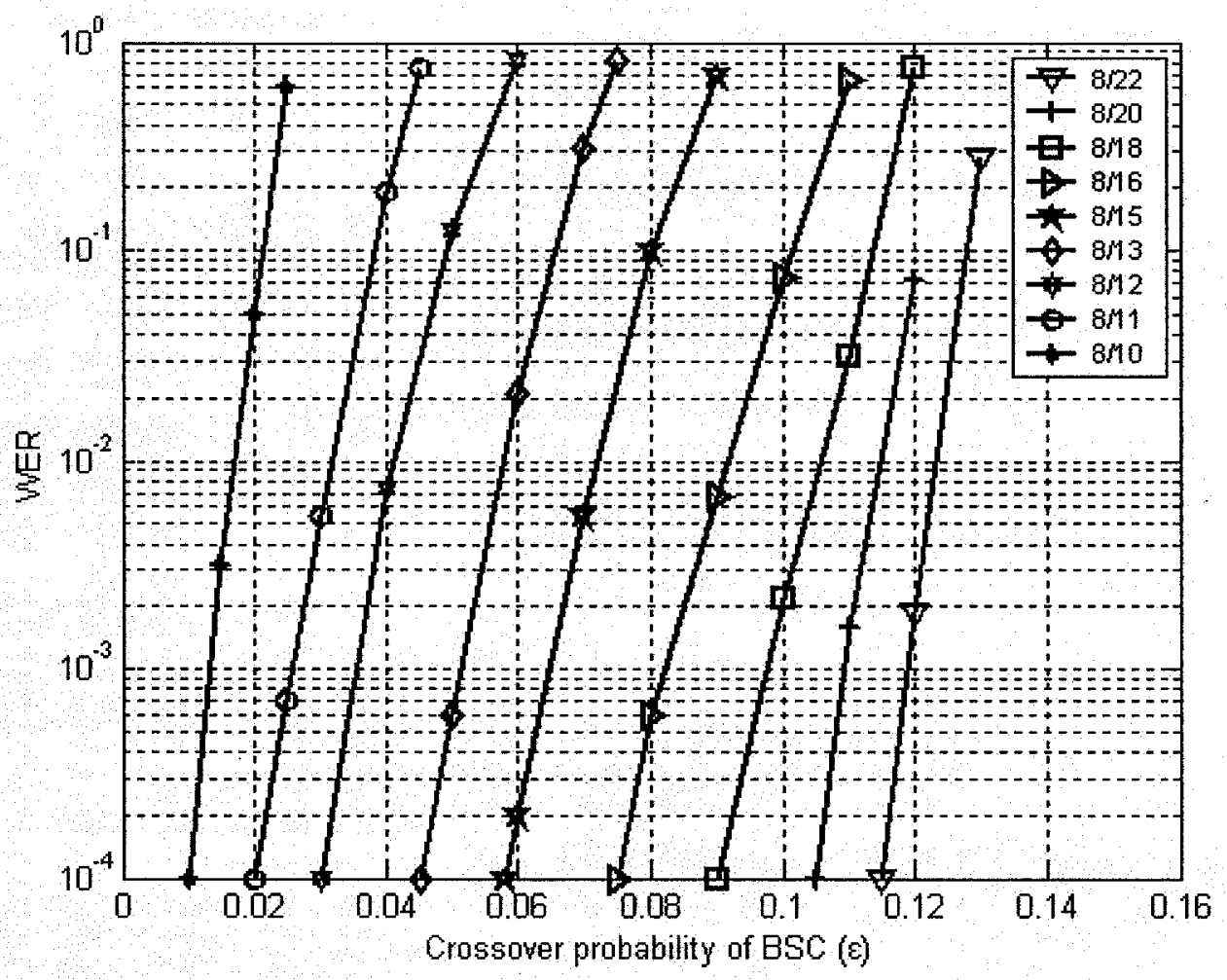

Figure 3.2: The WER curves of the channel codes over a BSC channel

As one can see in Figure 3.3, the nine curves are very close for any given value of $\varepsilon$. Accordingly, we use the average of the nine curves as the estimate $\hat{\alpha}(\varepsilon)$. The solid line in Figure 3.3 represents this average. Using Figure 3.3, we conservatively choose $\eta_{l}=\eta_{u}=$ 0.015 . Using these values, the range of (3.2) for $N^{*}$, which is equal to $\left(\eta_{I}+\eta_{u}\right) B / k$, is upper bounded by 2.54 for the image sizes and the total bit budgets under consideration. (This upper bound is obtained by $B=0.994 \times 512 \times 512$.) Thus, the trellis levels that contain the final candidate nodes are limited to at most only three regardless of the image, the total bit budget and the channel parameter. Note that although the actual levels do de- 
pend on the channel parameter and the total bit budget they are independent of the image.

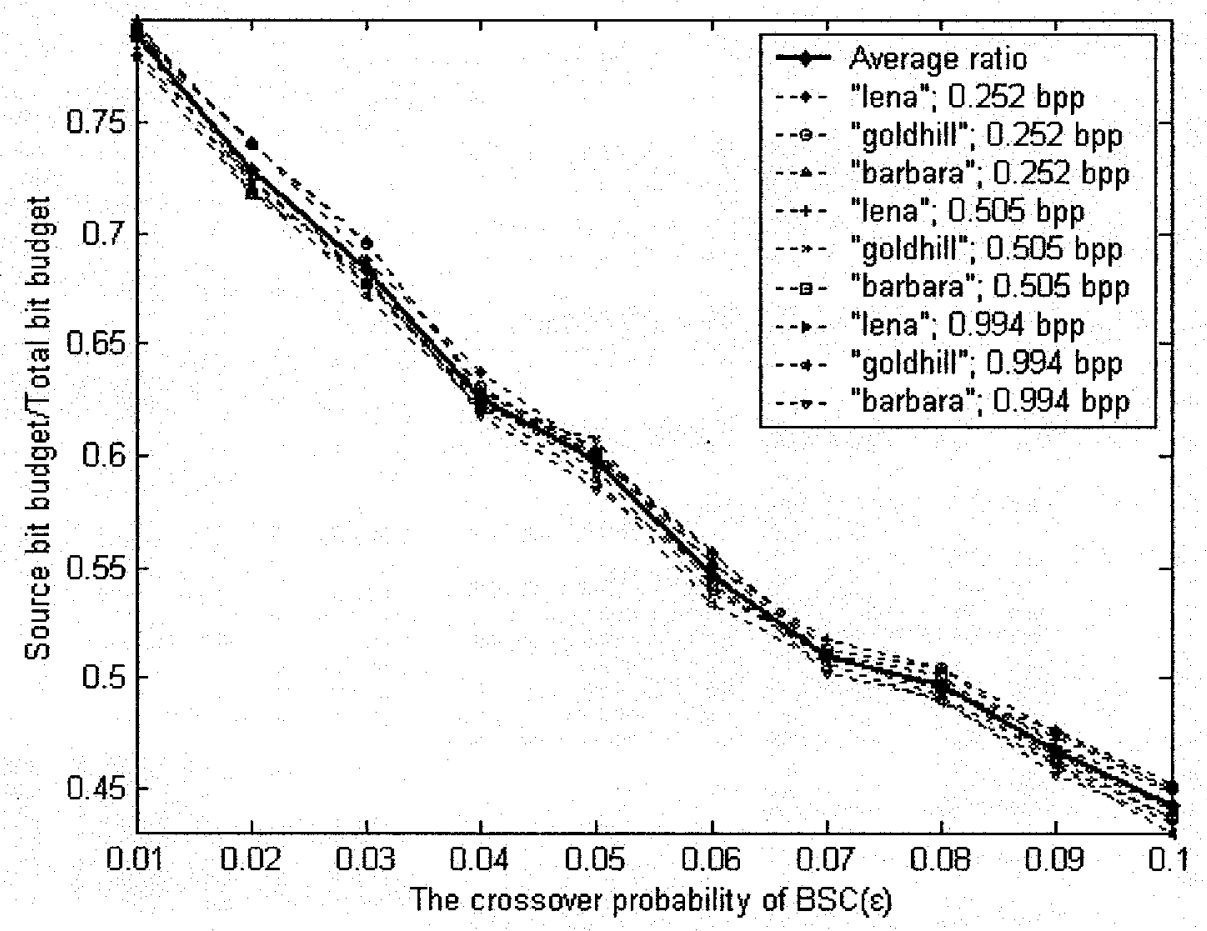

Figure 3.3: Optimal ratio of source bit budget to total bit budget for JPEG2000 algo-

rithm

If different bit budgets are used or if the channel parameter changes during the transmission of images, one may store the simplified trellises for different cases in memory and use them accordingly.

In our example, the application of Proposition 1 eliminates all but one final node at each level of the trellis. Application of (3.2) and Proposition 1 together reduces the number of branches on the search trellis by up to two orders of magnitude with no loss in performance. 
Table 3.1: Speed-up factors of the proposed rate-allocation algorithm compared with that of [2]

\begin{tabular}{|c|c|c|c|c|c|}
\hline \multirow{3}{*}{$\begin{array}{c}\text { Total Bit } \\
\text { Budget }\end{array}$} & \multicolumn{5}{|c|}{ Speed-up factor } \\
\cline { 2 - 6 } & 0.01 & 0.03 & 0.05 & 0.08 & 0.1 \\
\hline \hline $0.252 \mathrm{bpp}$ & 289.05 & 227.60 & 235.31 & 261.75 & 317.27 \\
\hline $0.505 \mathrm{bpp}$ & 364.26 & 249.67 & 277.84 & 329.19 & 467.79 \\
\hline $0.994 \mathrm{bpp}$ & 772.26 & 683.15 & 696.39 & 719.11 & 837.83 \\
\hline
\end{tabular}

Table 3.1 shows the speed-up factors of our algorithm compared with that of [2]. In addition to Proposition 1 and inequality (3.2), we have also incorporated the nondecreasing rate constraint and have allowed only $M^{\prime}(<M=10)$ rates for each node. As an example, Figure 3.2 shows that for $\varepsilon=0.08$, only the channel codes with rates $8 / 15,8 / 16$ and $8 / 18$ satisfy the constraints explained in the previous section and thus $M^{\prime}=3$ instead of 10 . Table 3.1 illustrates that our modified algorithm yields speed improvements up to a factor of 838 compared to the algorithm of [2]. As expected, the improvement increases by the total bit budget. Note that the improvement in speed is achieved at no cost in performance. In fact, for all the tested cases, our simplified trellis yields the same result as the one obtained from the full-search trellis.

In order to compare the contribution of (3.2) with that of the other three steps to the speed-up factor, we only apply the last three steps for simplifying the rate allocation scheme. As a result, we achieve speed-up factor of 39.76 for $0.994 \mathrm{bpp}$ and $\varepsilon$ of 0.1 compared to 837.83 .

To show the general applicability of our approach, we also tested $512 \times 5128$-bit monochrome images of "Peppers", "Boat", "Mandrill", "Zelda", and "Washsat" under 
different total bit budget constraints and channel conditions. In each case, the rateallocation result of our reduced search was the same as that of the full search.

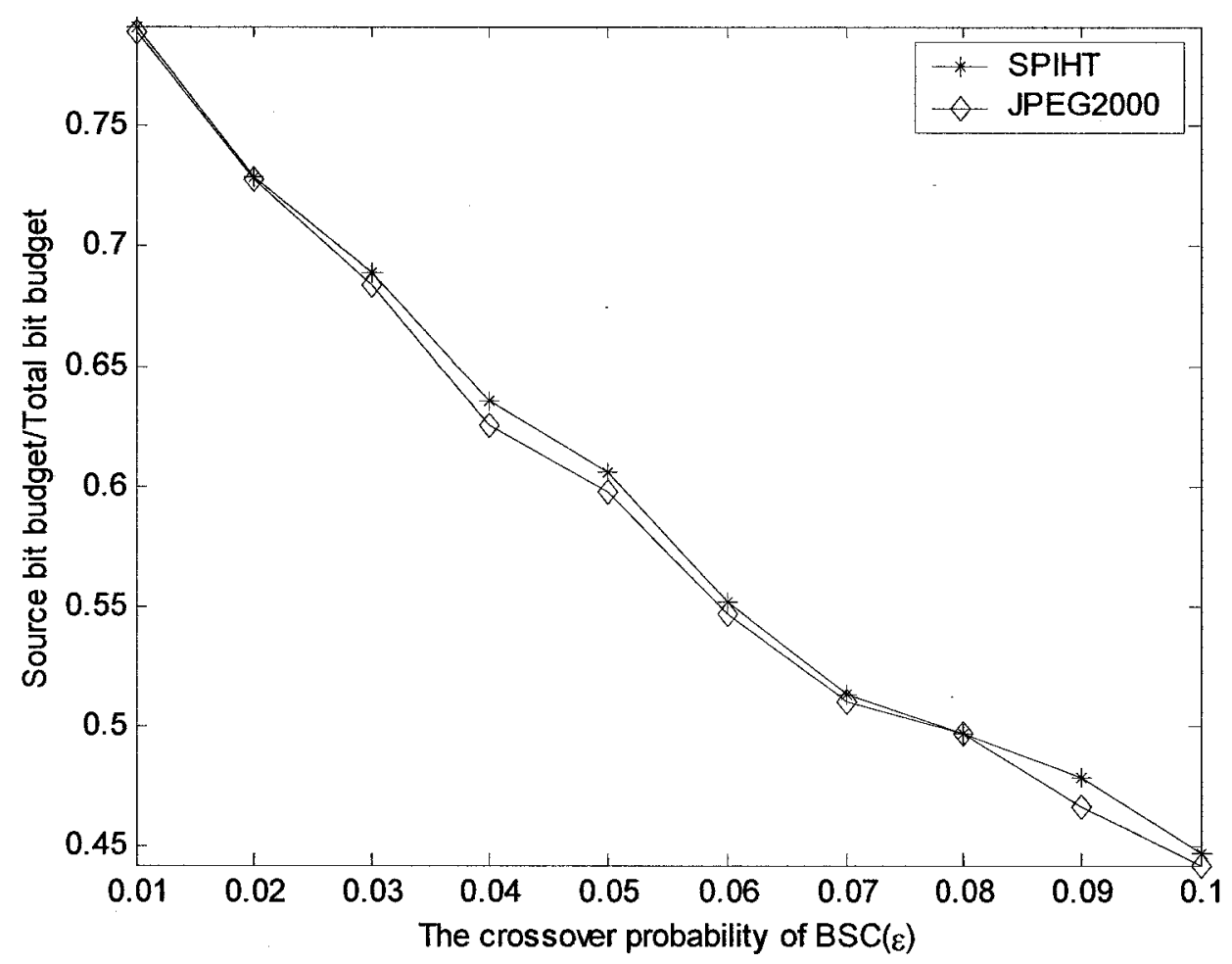

Figure 3.4: Average optimal ratio of source bit budget to total bit budget for JPEG2000 and SPIHT algorithms

We also repeated the same experiments using SPIHT as the source coding scheme and obtained similar results to those reported here. The average optimal ratio of source to total bit budget for SPIHT ( $\left.\hat{\alpha}(\varepsilon)_{\mathrm{SPIHT}}\right)$ is very close to that of JPEG2000 $\left(\hat{\alpha}(\varepsilon)_{\mathrm{JP} 2}\right)$, as demonstrated in Figure 3.4. Using $\hat{\alpha}(\varepsilon)=\left(\hat{\alpha}(\varepsilon)_{\mathrm{SPIHT}}+\hat{\alpha}(\varepsilon)_{\mathrm{JP} 2}\right) / 2$ and $\eta_{l}=\eta_{u}=0.018$, we may apply the same simplified search trellis to images compressed by either algorithm with no degradation of performance. 


\section{Chapter 4}

\section{Joint Source and Channel Coding}

\section{over BSC with FEC}

\subsection{Introduction}

Progressive image transmission [15], [16] and [17] is particularly desirable when transmitting images over internet and wireless channels since the bit stream can be decoded as it arrives, providing a continually improving approximation to the decoded data. However, such bit streams of the state-of-the-art progressive image coding algorithms are very sensitive to the bit errors in the presence of a noisy channel due to the propagation of errors at the decoder. As a result, channel coding should be employed to protect the bit stream against channel errors. As we discussed in Section 3.1, source and channel coding have been addressed as independent problems. However, when the constraints of practical communications systems are considered, it is often possible to find a better solution if an end-to-end framework in which the source/channel coding problem is solved jointly, is used. In such frameworks, typically, a rate allocation scheme is used to distribute the available total rate between the source and the channel encoders, in order to optimize 
some desired performance measure under a specific channel condition, see, e.g., [2], [3], $[15],[16]-[19]$.

Application of UEP to JPEG2000 bit streams over error-prone channels have been studied in [2], [16]-[20]. In particular, in [2], RCTCs have been employed and shown to provide improvements up to $1.6 \mathrm{~dB}$ in expected PSNR of reconstructed images over the similar results of [16] (based on RCPC codes). Lan et al [3] have further improved the results of [2] by using IRA codes. IRA codes are an example of LDPC codes [9]. In this work, we study the application of another construction of LDPC codes to the transmission of JPEG2000 compressed images.

To compare our results with those of [2] and [3], we make similar assumptions. In particular, we consider the transmission of JPEG2000 images over binary symmetric channels (BSC). We also use the same rate-allocation algorithm as the one in [2] and the same images as those in [2] and [3].

As we have discussed in Section 3.2, in our proposed scheme the JPEG2000 encoded bit stream is partitioned into blocks of equal length. Through the rate-allocation algorithm, each block will then be assigned a certain channel coding rate and will be encoded accordingly. Different transmitted packets will thus have different lengths. At the receiver, the received packets are decoded and for each packet if no error is detected, the information block is extracted for the reconstruction of the image. Following [2] and [3], we assume that when the first packet error is detected, the remaining packets following the erroneous one will be discarded even though they may be correctly decoded. This is done to avoid the propagation of error to the remaining packets. Furthermore, the data in one packet is considered as a whole, i.e., the whole packet will be discarded even if there is 
only one bit in error. This implies that in our study the word error rate (WER) performance of the channel codes is of main importance.

The construction of our RC-LDPC codes follows the same rules as those presented in Chapter 2.

\subsection{System Analysis}

Fig. 4.1 shows us the system structure of FEC system. The image after being encoded by JPEG2000 or SPIHT algorithm, becomes a progressively organized bitstream. The bitstream is simply partitioned into blocks with equal length. The rate-allocation algorithm then assigns the proper rates of RC-LDPC codes according to the distortions of the source packets. The channel coded bitstream is transmitted over the noisy channel. The corrupted bitstream is detected and recovered by the RC-LDPC decoder. Finally, the image is reconstructed using the recovered bitstreams.

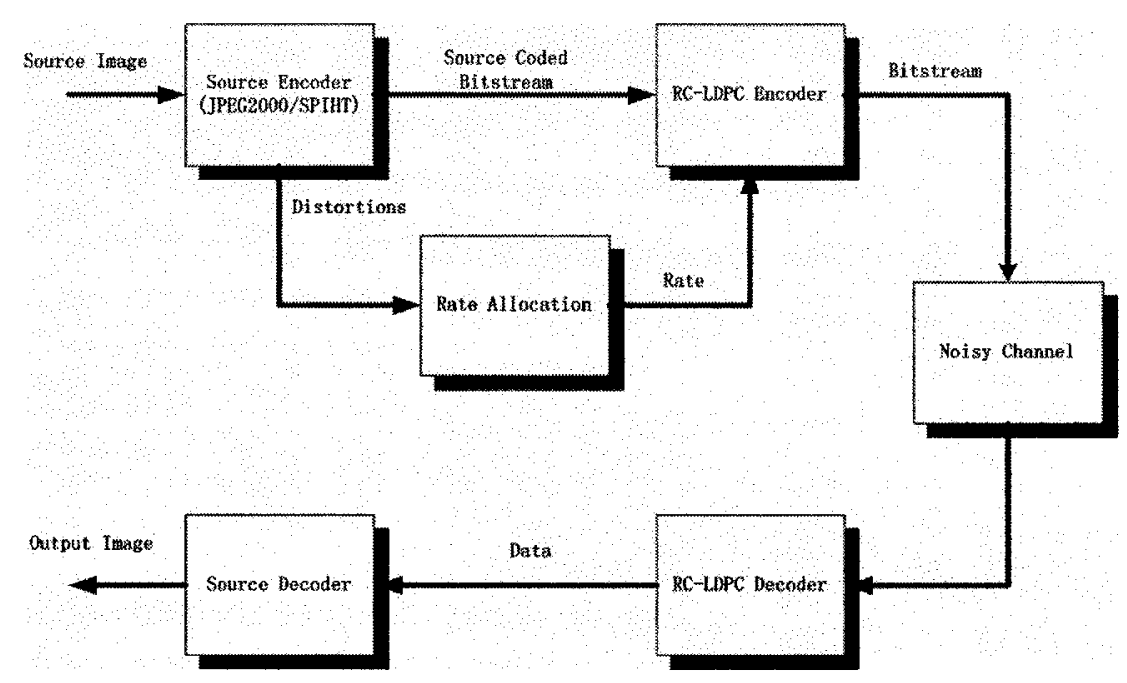

Figure 4.1: Block Diagram for FEC Scheme. 
We use the same method as in [1] to construct the parity check matrices of RC-LDPC codes. The information block length of the RC-LDPC codes, $k$, is chosen to be 384 bytes (3072 bits). This value is determined by simulation and appears to be optimal to maximize the PSNR of the recovered image for a wide range of total bit budgets and channel parameters. An example of the variation of PSNR against information block length is shown in Fig. 4.2 (a) for "Lena" when total bit budget is 0.252 bpp and $\varepsilon$ changes from 0.01 to 0.1 . It can be seen that for most of the curves the PSNR first increase with $k$. Then reach its top value at around 384 bytes. After that, the PSNR decline with $k$.

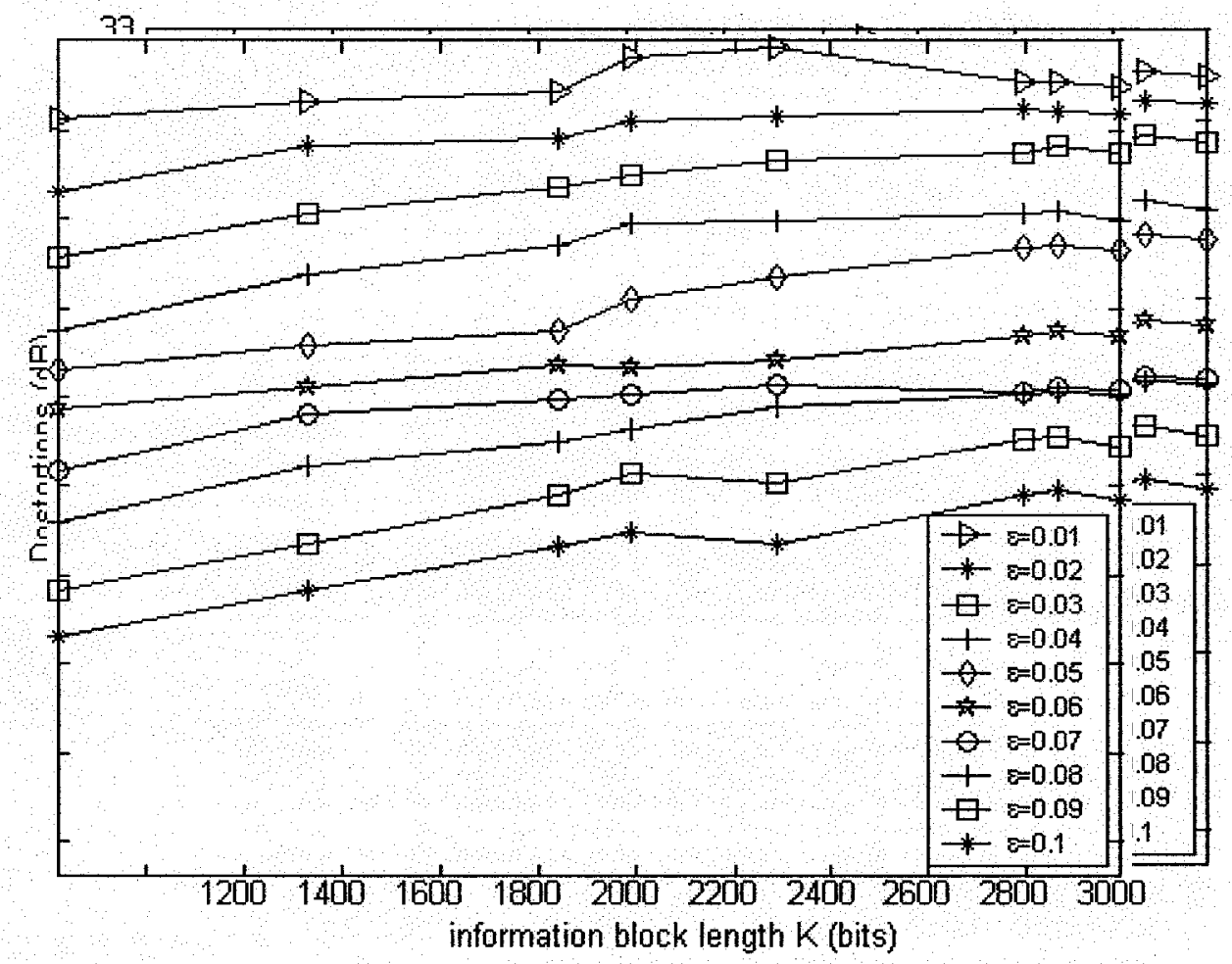

Figure 4.2 (a): PSNR vs. information block length ' $k$ ' for 'Lena", when total bit budget equals $0.252 \mathrm{bpp}$. 


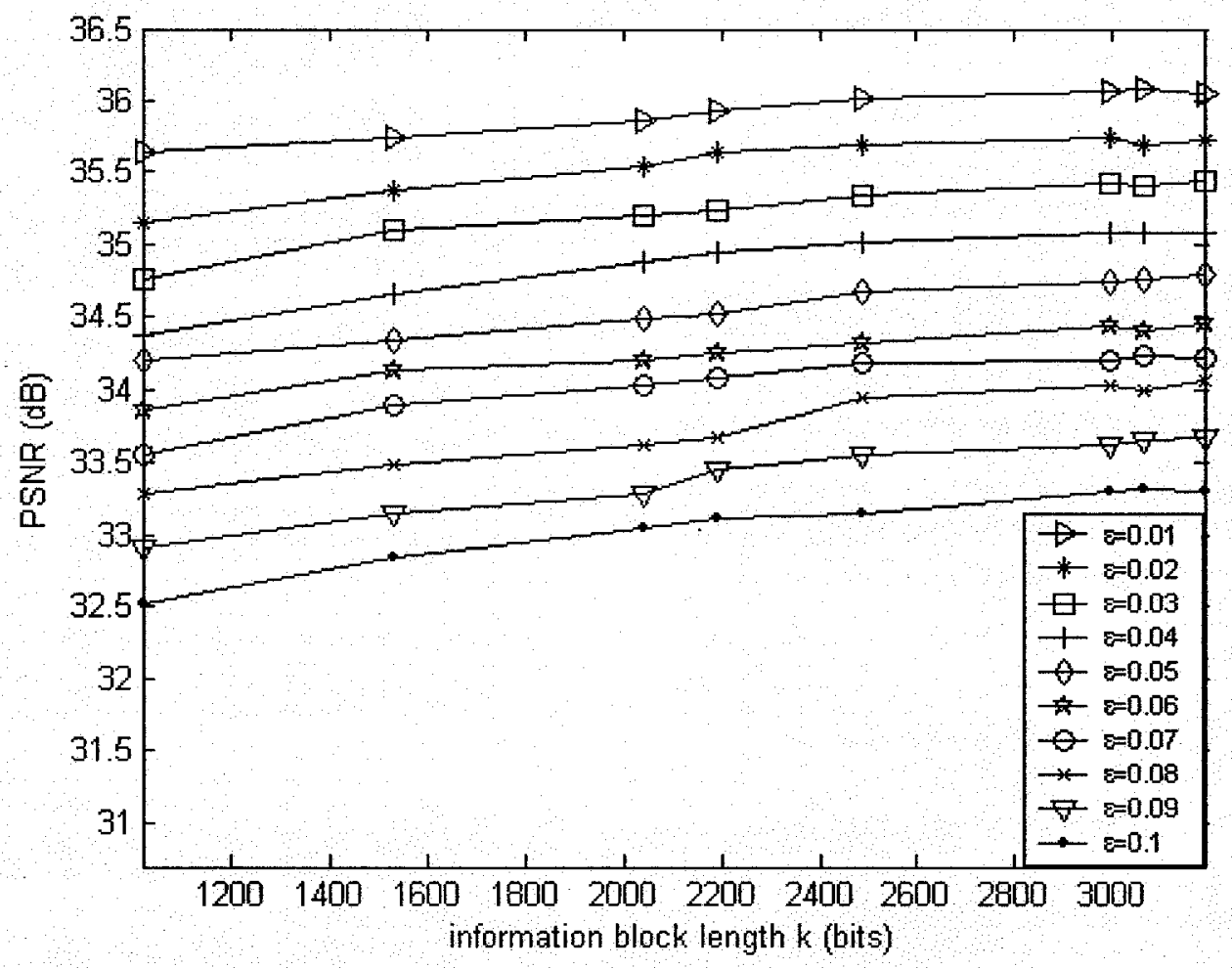

Figure 4.2 (b): PSNR vs. information block length ' $k$ ' for 'Lena", when total bit budget equals $0.505 \mathrm{bpp}$.

Also shown in Fig.4.2 (b) are the change of PSNR with ' $k$ ' for "Lena", when the total bit budget euuals $0.505 \mathrm{bpp}$. The length of $\mathrm{K}$ is chosen to achieve high PSNRs over a wide range of total bit bidget and channel parameters.

The parity check matrix of the mother code is of size $1920 \times 4992$. This corresponds to code rate $8 / 13$. The extension matrix $h_{\text {ext }}$ is of size $384 \times 384$. To generate parity-check matrices of our code sequence, nine matrices are obtained by extending the mother code and three by puncturing it (In order to do this, we have followed the same rules as in [1]. 
The general format of $H$ matrix of RC-LDPC codes is shown in Chapter 2. The $H$ matrix used in our FEC system over BSC is illustrated in Figure 4.3.

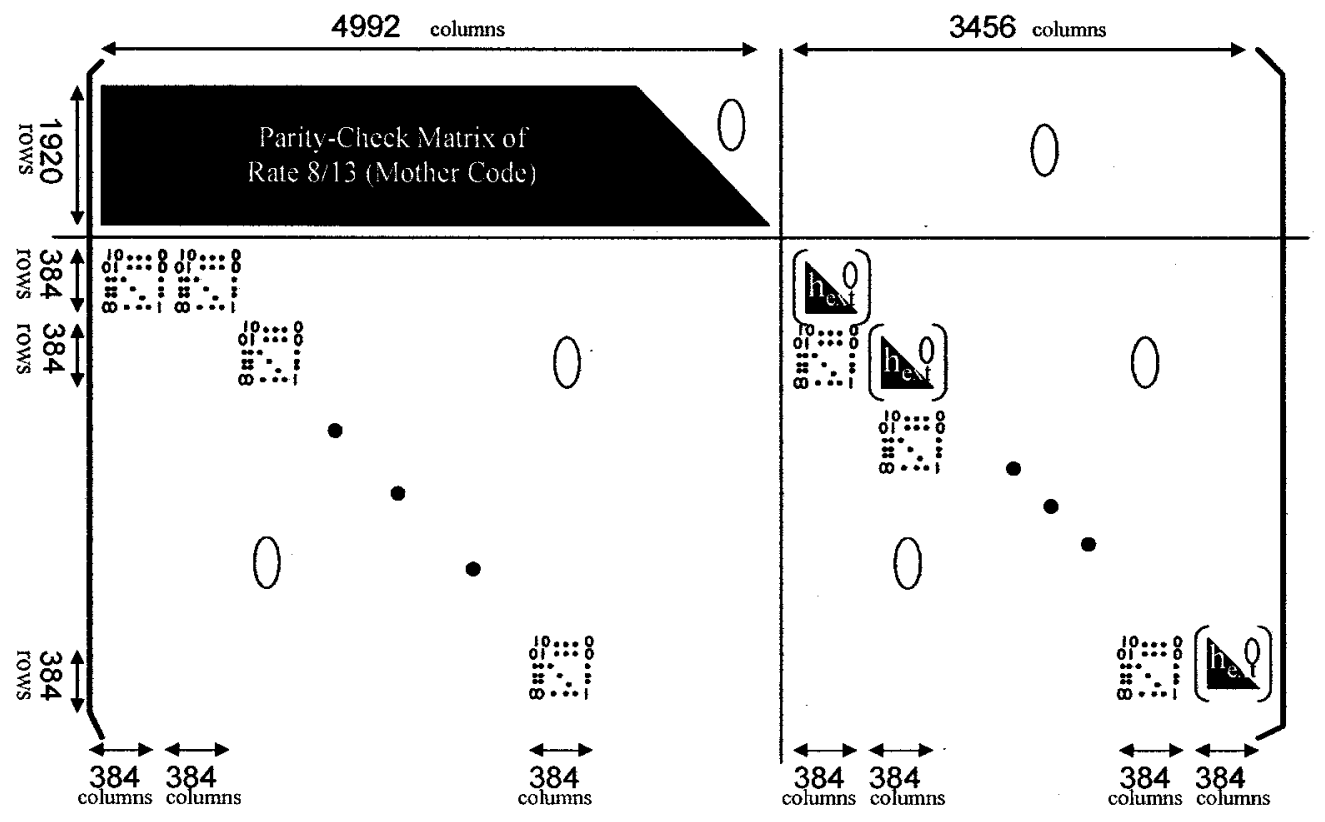

Figure 4.3: Construction of the parity-check matrices for the mother code and extended codes in the sequence of RC-LDPC codes.

Thus, the rates are $8 / 10,8 / 11,8 / 12, \ldots, 8 / 21,8 / 22$. To construct the mother code and $h_{e x t}$, similar to [10], we use linear-time PEG construction with the optimal symbol-node degree distribution. This degree distribution for a rate 0.5 code over $\mathrm{BSC}$ is given by [25]:

$$
\lambda(\mathrm{x})=0.3410084 \mathrm{x}^{2}+0.5538655 \mathrm{x}^{3}+0.10512598 \mathrm{x}^{11},
$$

where the powers of $x$ indicate the degrees of the symbol nodes and the coefficients of $x$ show the percentages of symbol nodes with those degrees.

From the available twelve rates, we select nine of them so that the corresponding WER curves all have roughly the same distance from their neighboring curves while covering 
the desirable range of crossover probabilities of BSC, i.e., from about 0.01 to about 0.1 . This uniform coverage by WER curves plays an important role in improving the quality of the reconstructed images for all the channel parameters in the range. The selected rates are: $8 / 10,8 / 11,8 / 12,8 / 13,8 / 15,8 / 16,8 / 18,8 / 20,8 / 22$, and the corresponding codeword lengths are: $480,528,576,624,720,768,864,960,1056$ (bytes), respectively. Including the rate $8 / 8$, we thus have ten rates available for channel coding. The WER curves for the codes are shown in Figure 4.4 by solid lines. In Figure 4.4, we have also included the performance curves of the RCTCs of [2]. As can be seen, the constructed RC-LDPC codes provide a more uniform coverage of the favorable range of channel parameters. Moreover, unlike the curves for turbo codes, the WER curves of LDPC codes show no sign of early error floor in the simulated range of WERs ${ }^{6}$. Similarly, compared to the IRA codes of [3], the constructed codes perform better and also provide a more uniform coverage of the favorable range of channel parameters.

For measuring the performance of the combined source and channel coding scheme, we use the expected PSNR, and our goal is to maximize it. This is accomplished by minimizing the expected distortion of the reconstructed image. If the compressed bit stream of the source image is divided into $N$ packets for transmission, the expected distortion in Mean Squared Error (MSE) can be calculated using the (3.1) given in Chapter 3.

The bit stream generated by JPEG2000 encoder is first partitioned into packets of equal length L (383 bytes). An extra byte is added to each packet to inform the RC-LDPC de-

\footnotetext{
${ }^{6}$ Note that for rate 0.5 , the constructed LDPC code outperforms the corresponding turbo code by more than an order of magnitude in WER. This could however be at least partly due to the larger block length for the LDPC code ( 768 bytes vs. 517 bytes for the turbo code). It should be also noted that although for LDPC codes the block lengths for 8 out of 10 rates are larger than the block length for turbo codes of [2], for the transmission of an image, this is not a disadvantage neither from complexity nor from delay point of view. To verify this, one should note that the decoding complexity per coded bit for LDPC codes is constant and the total transmitted bits (total bit budget) is also a fixed value regardless of the block lengths used. The total delay for the reconstruction of the image would be dominated by the delay in transmission of packets, which mainly depends on the transmission rate and is independent of the block lengths.
} 
coder about the channel coding rate used for the next packet (The information block length $k$ is thus equal to $\mathrm{L}+1$ bytes). The resulting packets are then coded by RC-LDPC codes using different rates. Depending on the channel parameter, the lowest required rate, known to both the transmitter and the receiver based on WER curves, is assigned to the first packet. When the first packet is correctly decoded, the channel decoder will obtain the channel coding rate and thus the block length for the next packet. This process continues until an error is detected. ${ }^{7}$ At such point, the channel decoder ignores the rest of the packets.

To determine the partitioning of the total bit budget between the source and the channel encoders and to find the channel coding rate for each packet (other than the first one), we use a similar rate-allocation algorithm as the one in [2]. With a constraint on the total bit budget, this algorithm minimizes the expected distortion of (3.1) for a given channel parameter $\varepsilon$.

\subsection{Performance of the FEC Scheme over}

\section{BSC}

The WER comparison of RC-LDPC codes and RCTC are given in Figure 4.4. They are obtained using iterative belief propagation algorithm over BSC [12]. The maximum

\footnotetext{
${ }^{7}$ Since the RC-LDPC codes have the capability of detecting errors, it is unnecessary to use a cyclic redundancy check (CRC) code for error detection. The saved bits can then be used for source encoding to improve the quality of image. This is another advantage of RCLPDC codes over RCTCs. The error detection capability of RC-LDPC codes has been verified by our simulations.
} 
number of iterations is set to $500,{ }^{8}$ and similar to [2], 10,000 code words are simulated for each channel parameter. As WERs below $10^{-4}$ are effectively equivalent to zero error probability in calculation of PSNR, the curves have been only plotted down to $10^{-4}$.

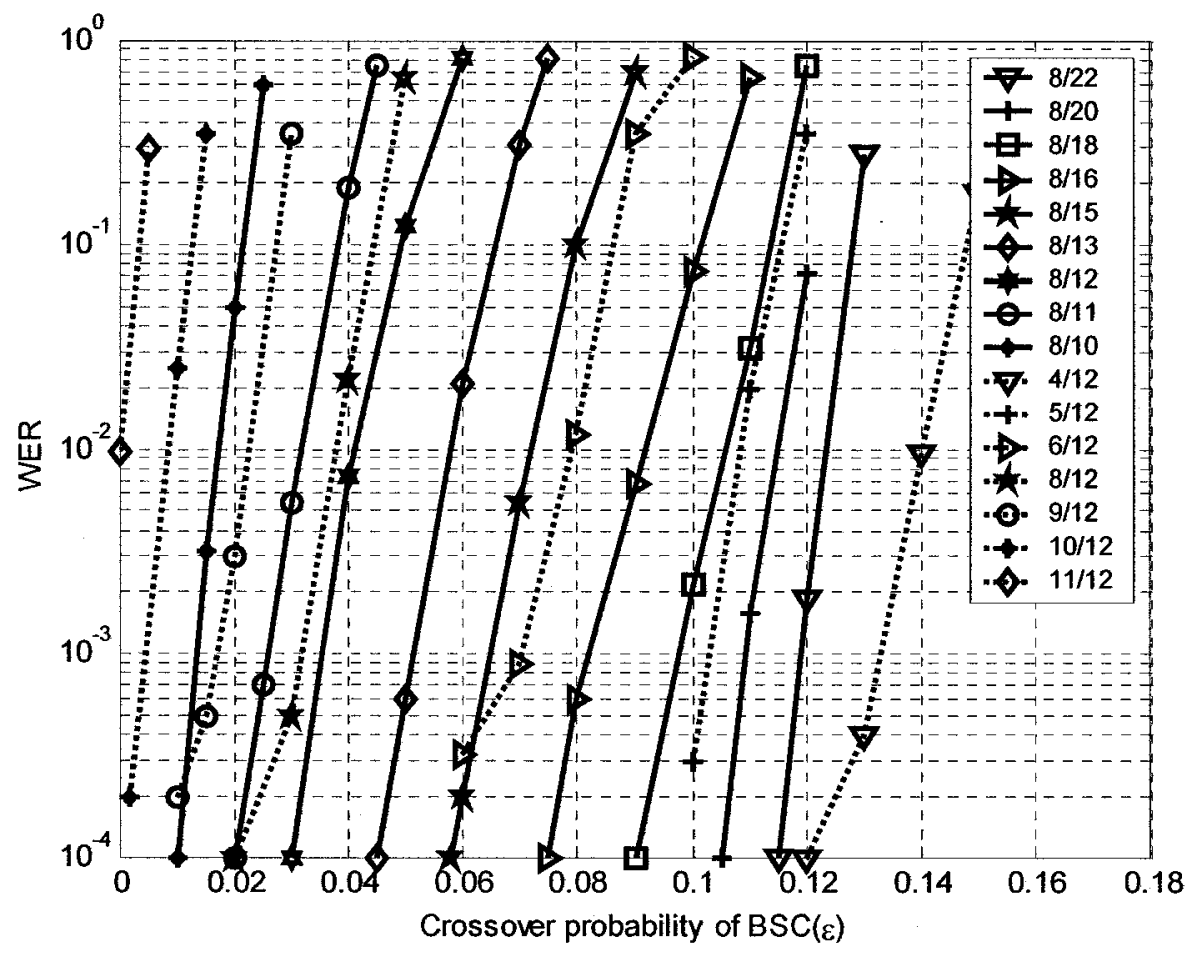

Figure 4.4: Performance of RC-LDPC codes with information block length $K=384$ bytes at different rates (solid lines); Performance of RCTCs of [2] with block length $=517$ bytes (dotted lines).

To compare our results with those of [2] and [3], we use the same set of images as in [2] and [3]: 512×512 8-bit monochrome "Lena", "Goldhill" (from USC database) and "Barbara" [26]. These images are encoded by JPEG2000 encoder using default options.

\footnotetext{
${ }^{8}$ In practice, the maximum number of iterations does not need to be this large. In fact, by reducing this number to 100 , the obtained PSNRs, reported in Table 4.3, will only be reduced by up to about $0.1 \mathrm{~dB}$.
} 
For JPEG2000 codec, we use the software provided in [27], [28]. As a fair comparison with the results in [2], we first test our system for the capacity values and obtain the same results as those of [2]. The distortion values required for the rate-allocation algorithm are obtained using the method explained in Section 2.1.2

The expected PSNRs for the total rates of $0.252,0.505$ and $0.994 \mathrm{bpp}$, and channel crossover probabilities ranging from 0.01 to 0.1 are shown in Table 4.1. For comparison, the corresponding results from [2] and [3] are also listed in Table 4.1. As expected, PSNR increases with the increase of the total rate and decreases with the increase of the channel parameter. As can be seen in Table 4.1, our results outperform the results of [2] consistently, and those of [3] in all but four cases. For these four cases, our scheme has a degradation of up to only about $0.1 \mathrm{~dB}$. The largest improvement of our scheme over [2] is $1.09 \mathrm{~dB}$ which occurs for "Lena" at total rate of $0.252 \mathrm{bpp}$ and channel parameter $\varepsilon=$ 0.08. Compared to [3], the largest improvement is $0.98 \mathrm{~dB}$ which happens for "Barbara" at total rate of $0.994 \mathrm{bpp}$ and $\varepsilon=0.08$.

As an upper bound on expected PSNR, for the three images, we have listed in Table 4.2 the source PSNR at the ideal rate equal to the total rate times the capacity of the channel. This is the PSNR for the reconstructed image assuming that one can transmit information over the channel error-free at a rate equal to the capacity of the channel. The results are given for the total rate of $0.994 \mathrm{bpp}$ and channel parameters $0.01,0.03,0.08$ and 0.1 . It can be seen that the gaps to the upper bound have been greatly decreased compared to RCTCs and IRA codes. The smallest gap is now $0.81 \mathrm{~dB}$ which occurs for "Lena" at the channel parameter of 0.01 . 
Table 4.1: Expected PSNR (dB) for RC-LDPC codes, RCTCs and IRA Codes

\begin{tabular}{|c|c|c|c|c|c|c|}
\hline \multirow{2}{*}{ Image } & \multirow{2}{*}{ Total Rate } & \multirow{2}{*}{$\begin{array}{c}\text { Channel } \\
\text { Code } \\
\end{array}$} & \multicolumn{4}{|c|}{ Channel BER $(\varepsilon)$} \\
\hline & & & 0.01 & 0.03 & 0.08 & 0.1 \\
\hline \multirow{9}{*}{ Lena } & \multirow{3}{*}{0.252 bpp } & RC-LDPC & 32.77 & 32.42 & 31.03 & 30.48 \\
\hline & & $\begin{array}{c}\text { RCTC } \\
\text { ([2]) }\end{array}$ & 32.56 & 31.90 & 29.94 & 29.40 \\
\hline & & IRA ([3]) & 32.75 & 32.09 & 30.24 & 29.92 \\
\hline & \multirow{3}{*}{$0.505 \mathrm{bpp}$} & RC-LDPC & 36.08 & 35.41 & 34.00 & 33.32 \\
\hline & & $\begin{array}{c}\text { RCTC } \\
\text { ([2]) }\end{array}$ & 35.67 & 35.15 & 33.20 & 32.76 \\
\hline & & IRA ([3]) & 36.18 & 35.48 & 33.37 & 33.13 \\
\hline & \multirow{3}{*}{0.994 bpp } & RC-LDPC & 39.03 & 38.34 & 36.91 & 36.19 \\
\hline & & $\begin{array}{c}\text { RCTC } \\
([2])\end{array}$ & 38.78 & 37.74 & 36.15 & 35.85 \\
\hline & & IRA ([3]) & 38.93 & 38.26 & 36.26 & 36.03 \\
\hline \multirow{9}{*}{ Goldhill } & \multirow{3}{*}{0.252 bpp } & RC-LDPC & 29.79 & 29.35 & 28.49 & 28.09 \\
\hline & & $\begin{array}{c}\text { RCTC } \\
\text { ([2]) }\end{array}$ & 29.64 & 29.16 & 27.88 & 27.69 \\
\hline & & IRA ([3]) & 29.74 & 29.23 & 28.01 & 27.90 \\
\hline & \multirow{3}{*}{$0.505 \mathrm{bpp}$} & RC-LDPC & 32.28 & 31.65 & 30.52 & 30.13 \\
\hline & & $\begin{array}{c}\text { RCTC } \\
([2])\end{array}$ & 31.79 & 31.38 & 30.04 & 29.89 \\
\hline & & IRA ([3]) & 32.10 & 31.65 & 30.12 & 29.99 \\
\hline & \multirow{3}{*}{$0.994 \mathrm{bpp}$} & RC-LDPC & 35.23 & 34.33 & 32.99 & 32.42 \\
\hline & & $\begin{array}{c}\text { RCTC } \\
\text { ([2]) }\end{array}$ & 34.81 & 34.05 & 32.24 & 32.10 \\
\hline & & IRA ([3]) & 35.00 & 34.18 & 32.30 & 32.20 \\
\hline \multirow{9}{*}{ Barbara } & \multirow{3}{*}{$0.252 \mathrm{bpp}$} & RC-LDPC & 27.31 & 26.69 & 25.45 & 25.14 \\
\hline & & $\begin{array}{c}\text { RCTC } \\
([2])\end{array}$ & 26.93 & 26.32 & 24.84 & 24.71 \\
\hline & & IRA ([3]) & 27.00 & 26.37 & 24.89 & 24.73 \\
\hline & \multirow{3}{*}{05 bpp } & RC-LDPC & 30.79 & 29.91 & 28.39 & 27.63 \\
\hline & & $\begin{array}{c}\text { RCTC } \\
([2]) \\
\end{array}$ & 30.35 & 29.41 & 27.54 & 26.99 \\
\hline & & IRA ([3]) & 30.84 & 29.88 & 27.66 & 27.42 \\
\hline & \multirow{3}{*}{94 bpp } & RC-LDPC & 35.02 & 33.93 & 31.83 & 30.91 \\
\hline & & $\begin{array}{c}\text { RCTC } \\
\text { ([2]) }\end{array}$ & 34.40 & 33.54 & 30.82 & 30.51 \\
\hline & & IRA ([3]) & 34.89 & 33.74 & 30.85 & 30.77 \\
\hline
\end{tabular}


Table 4.2: Expected PSNR (dB) for the proposed schemes based on RC-LDPC codes, RCTCs and IRAs, and for the error-free transmission at capacity rate (Total rate $=0.994$ bpp)

\begin{tabular}{|c|c|c|c|c|c|c|c|c|c|c|c|c|c|}
\hline \multirow{3}{*}{ Image } & \multirow{3}{*}{ Comparison } & \multicolumn{12}{|c|}{ Channel BER } \\
\hline & & \multicolumn{3}{|c|}{0.01} & \multicolumn{3}{|c|}{0.03} & \multicolumn{3}{|c|}{0.08} & \multicolumn{3}{|c|}{0.1} \\
\hline & & $\begin{array}{c}\text { RC- } \\
\text { LDPC } \\
\end{array}$ & $\begin{array}{c}\text { RCTC } \\
{[2]} \\
\end{array}$ & $\begin{array}{c}\text { IRA } \\
{[3]} \\
\end{array}$ & $\begin{array}{c}\text { RC- } \\
\text { LDPC } \\
\end{array}$ & $\begin{array}{c}\text { RCTC } \\
{[2]} \\
\end{array}$ & $\begin{array}{c}\text { IRA } \\
{[3]} \\
\end{array}$ & $\begin{array}{c}\mathrm{RC}- \\
\mathrm{LDPC} \\
\end{array}$ & $\begin{array}{c}\text { RCTC } \\
{[2]} \\
\end{array}$ & $\begin{array}{r}\text { IRA } \\
{[3]} \\
\end{array}$ & $\begin{array}{c}\mathrm{RC}- \\
\mathrm{LDPC} \\
\end{array}$ & $\begin{array}{c}\mathrm{RCTC} \\
{[2]} \\
\end{array}$ & $\begin{array}{c}\text { IRA } \\
{[3]} \\
\end{array}$ \\
\hline \multirow{3}{*}{ Lena } & Proposed & 39.03 & 38.78 & 38.93 & 38.34 & 37.74 & 38.26 & 36.91 & 36.15 & 36.26 & 36.19 & 35.85 & 36.03 \\
\hline & At Capacity & \multicolumn{3}{|c|}{39.84} & \multicolumn{3}{|c|}{39.24} & \multicolumn{3}{|c|}{37.90} & \multicolumn{3}{|c|}{37.36} \\
\hline & Gap & 0.81 & 1.06 & 0.91 & 0.90 & 1.50 & 0.98 & 0.99 & 1.75 & 1.64 & 1.17 & 1.51 & 1.33 \\
\hline \multirow{3}{*}{ Goldhill } & Proposed & 35.23 & 34.81 & 35.00 & 34.33 & 34.05 & 34.18 & 32.99 & 32.24 & 32.30 & 32.42 & 32.1 & 32.20 \\
\hline & At Capacity & \multicolumn{3}{|c|}{36.06} & \multicolumn{3}{|c|}{35.40} & \multicolumn{3}{|c|}{33.92} & \multicolumn{3}{|c|}{33.39} \\
\hline & Gap & 0.83 & 1.25 & 1.06 & 1.07 & 1.35 & 1.22 & 0.93 & 1.68 & 1.62 & 0.97 & 1.29 & 1.19 \\
\hline \multirow{3}{*}{ Barbara } & Proposed & 35.02 & 34.40 & 34.89 & 33.93 & 33.54 & 33.74 & 31.83 & 30.82 & 30.85 & 30.91 & 30.51 & 30.77 \\
\hline & At Capacity & \multicolumn{3}{|c|}{36.46} & \multicolumn{3}{|c|}{35.29} & \multicolumn{3}{|c|}{33.32} & \multicolumn{3}{|c|}{32.45} \\
\hline & Gap & 1.44 & 2.06 & 1.57 & 1.36 & 1.75 & 1.55 & 1.49 & 2.50 & 2.47 & 1.54 & 1.94 & 1.68 \\
\hline
\end{tabular}

In Table 4.3, we give the average and the standard deviation of the number of iterations required for convergence of each packet for different images, total bit rates and channel parameters. As can be seen, the iterative decoding algorithm on average requires only a small number of iterations to converge (between 20 and 37; much smaller than 500 ). The decoding speed and complexity are therefore comparable with those of the IRA codes used in [3] and better than those of the turbo codes of [2] (It is shown in [3] that the decoding complexity of IRA codes is lower than that of turbo codes of [2]). It is also worth mentioning that while our proposed codes are truly rate-compatible and therefore a single decoder can be used to decode them all, this is not the case for the IRA codes of [3]. 
Table 4.3: Statistics (mean and standard deviation) of the number of iterations required for the convergence of each RC-LDPC coded packet

\begin{tabular}{|c|c|c|c|c|c|c|c|c|c|}
\hline \multirow{3}{*}{ Image } & \multirow{3}{*}{ Total Rate } & \multicolumn{8}{|c|}{ Channel BER ( $(\varepsilon)$} \\
\hline & & \multicolumn{2}{|c|}{0.01} & \multicolumn{2}{|c|}{0.03} & \multicolumn{2}{|c|}{0.08} & \multicolumn{2}{|c|}{0.1} \\
\hline & & Mean & $\begin{array}{c}\text { Standard } \\
\text { Deviation }\end{array}$ & Mean & $\begin{array}{c}\text { Standard } \\
\text { Deviation }\end{array}$ & Mean & $\begin{array}{c}\text { Standard } \\
\text { Deviation }\end{array}$ & Mean & $\begin{array}{l}\text { Standard } \\
\text { Deviation }\end{array}$ \\
\hline \multirow{3}{*}{ Lena } & $0.252 \mathrm{bpp}$ & 24.73 & 23.25 & 32.04 & 21.31 & 30.91 & 20.14 & 23.54 & 28.64 \\
\hline & $0.505 \mathrm{bpp}$ & 24.71 & 22.50 & 29.12 & 19.68 & 28.37 & 17.52 & 20.37 & 24.33 \\
\hline & $0.994 \mathrm{bpp}$ & 22.83 & 25.19 & 25.15 & 21.57 & 36.56 & 19.64 & 20.82 & 18.75 \\
\hline \multirow{3}{*}{ Goldhill } & $0.252 \mathrm{bpp}$ & 24.73 & 23.25 & 32.04 & 21.31 & 30.91 & 20.14 & 23.54 & 28.64 \\
\hline & 0.505 bpp & 24.71 & 22.50 & 29.09 & 16.75 & 28.37 & 17.52 & 29.67 & 21.48 \\
\hline & $0.994 \mathrm{bpp}$ & 25.47 & 26.23 & 27.21 & 23.17 & 36.56 & 20.47 & 20.46 & 20.44 \\
\hline \multirow{3}{*}{ Barbara } & 0.252 bpp & 24.73 & 23.25 & 32.04 & 21.31 & 30.91 & 20.14 & 23.54 & 28.64 \\
\hline & $0.505 \mathrm{bpp}$ & 24.71 & 22.50 & 29.04 & 18.79 & 33.21 & 18.05 & 29.67 & 21.48 \\
\hline & $0.994 \mathrm{bpp}$ & 26.43 & 26.55 & 25.21 & 27.01 & 36.56 & 25.88 & 20.46 & 20.44 \\
\hline
\end{tabular}

\subsection{Estimation Error for Channel Parameter}

Table 4.4 contains expected PSNR results when there is a mismatch between the actual and the estimated crossover probability of BSC. Again following [2], the results are given

Table 4.4: Expected PSNR (dB) with mismatched BERs (Total rate $=0.994 \mathrm{bpp})$

\begin{tabular}{|c|c|c|c|c|c|c|c|c|}
\hline \multirow{3}{*}{ Image } & \multicolumn{8}{|c|}{ Estimated Channel BER/Actual Channel BER } \\
\hline & \multicolumn{2}{|c|}{$0.01 / 0.03$} & \multicolumn{2}{|c|}{$0.03 / 0.01$} & \multicolumn{2}{|c|}{$0.08 / 0.1$} & \multicolumn{2}{|c|}{$0.1 / 0.08$} \\
\hline & $\begin{array}{c}\mathrm{RC}- \\
\mathrm{LDPC}\end{array}$ & $\begin{array}{c}\text { RCTC } \\
{[2]}\end{array}$ & $\begin{array}{c}\text { RC- } \\
\text { LDPC }\end{array}$ & $\begin{array}{c}\mathrm{RCTC} \\
{[2]}\end{array}$ & $\begin{array}{c}\mathrm{RC}- \\
\mathrm{LDPC}\end{array}$ & $\begin{array}{c}\mathrm{RCTC} \\
{[2]}\end{array}$ & $\begin{array}{c}\text { RC- } \\
\text { LDPC }\end{array}$ & $\begin{array}{c}\mathrm{RCTC} \\
{[2]}\end{array}$ \\
\hline Lena & 30.99 & 30.28 & 38.49 & 37.78 & 35.82 & 33.69 & 36.24 & 35.88 \\
\hline Goldhill & 25.65 & 24.26 & 34.42 & 34.17 & 32.48 & 32.14 & 32.52 & 32.13 \\
\hline Barbara & 26.07 & 25.51 & 34.12 & 33.65 & 30.19 & 27.91 & 31.17 & 30.55 \\
\hline
\end{tabular}

for the total rate of $0.994 \mathrm{bpp}$, and for four different mismatch scenarios. We also listed the results of [2] for comparison. It can be seen that in general, underestimation of the 
channel parameter is more deteriorating than overestimation, and can result in a few $\mathrm{dB}$ loss in PSNR. This is due to the fact that when the channel parameter is underestimated the rate-allocation algorithm assigns higher rates than what is really needed to properly protect the packets. This under-protection of the packets, especially the more important ones at the beginning of the bit stream, will result in larger error rates and smaller PSNRs. Still, one should note that for all the cases, our scheme outperforms that of [2], with the largest improvement equal to $2.28 \mathrm{~dB}$ which occurs for "Barbara" when the estimated and actual channel parameters are equal to 0.08 and 0.1 , respectively. 


\section{Chapter 5}

\section{Progressive Image Transmission}

\section{over Fading Channels with FEC}

\subsection{Introduction}

In the previous chapter we investigated a JSCC scheme using RC-LDPC codes over BSC. In this chapter we present the application of the proposed JSCC scheme to the fading channels.

In a FEC communication system, fading channels without feedback are investigated in $[5],[6],[15],[18]$ and $[29]-[32]$ for a variety of fading channel models. In [15], the fading channel is simulated using a Markov model. Later in [29], a combined framework incorporating SPIHT source encoder with CRC/RCPC channel codes is proposed for fading channels which are modeled by Gilbert-Elliott two-state model. The fading channel in [30] is modeled as a combination of a packet erasure channel followed by a discrete channel with memory. Sanchez et al. [18] use CRC/RCTC scheme over Rayleigh fading channels for JPEG2000 coded images. Hybrid channel coding and packetization schemes for image transmission is proposed in [31], where the fading channel is again modeled as 
a Gilbert-Elliott two-state model. In [32], Stankovic defines the fading channel as a packet erasure channel. They simulate the channel both with a two-state Markov packet loss model and a concatenation of a packet erasure channel with a BSC channel.

In this chapter, we use the same fading model as those of [5] and [6], where channel is modeled with Rayleigh flat fading model. Stankvoic et al. [5] propose the protection of SPIHT coded images using product codes. EEP is applied by combining CRC and RCPC codes with Read-Solomon (RS) codes. They achieve great improvement over [30]. EEP of [5] perform better than UEP at small SNR. However, when channel SNR is not that small, UEP achieve higher PSNR than EEP. Our proposed FEC scheme with RC-LDPC codes outperforms those of [5] and [6] over a wide range of channel SNR. To be more specific, our scheme could achieve comparable or better PSNR values than those [5] at small channel SNR and could get higher PSNR than those of [6] at large channel SNR.

\subsection{System Analysis}

The RC-LDPC codes are constructed using the technique given in Chapter 2. The source encoded bitstream is transmitted using the FEC scheme, which is described in Chapter 4. At the transmitter, our fast trellis-based rate-allocation algorithm is applied to assign the optimal channel code rates. In this chapter, compressed images are transmitted over fading channels (Rayleigh flat-fading channels). The channel model is presented in the next section. 


\subsubsection{Rayleigh Fading Channel Model}

In our work, we will examine our scheme over both uncorrelated and correlated Rayleigh flat-fading channels. We assume that the channel is a discrete-time Rayleigh flat-fading channel. The Rayleigh fading channel can be modeled as shown Figure 5.1.

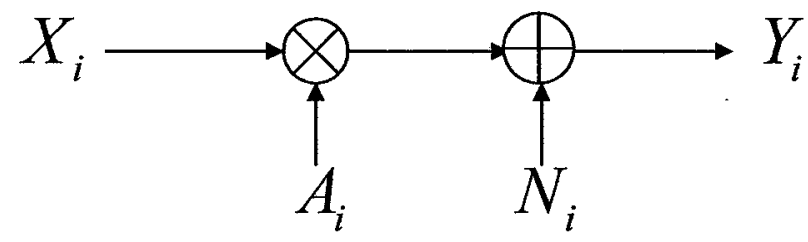

Figure 5.1: Rayleigh fading model

The output $Y_{i}$ of the channel is:

$$
Y_{i}=A_{i} X_{i}+N_{i}
$$

where $X_{i}$ is the channel input; $N_{i}$ is Gaussian random variable with zero mean and variance $\sigma_{N}^{2} ; A_{i}$ is a random variable with zero mean and variance $\sigma_{A_{i}}^{2}$. All the random variables $A_{i}, X_{i}$ and $N_{i}$ 's are independent. The amplitude of the fading coefficients $A_{i}$ is Rayleigh distributed with $E\left[A_{i}{ }^{2}\right]=1$ and density function $p\left(A_{i}\right)=2 A_{i} \exp \left(-A_{i}{ }^{2}\right)$.

At the LDPC decoder, the initial messages have to be set before applying iterative decoding algorithm. For Rayleigh fading channel with side information (SI), the LDPC decoder knows the ideal SI and the exact values of $A_{i}$ 's are available to the decoder. Binary Phase Shift Keying (BPSK) is used in our system, where 1 and 0 are mapped to -1 and 1 , respectively. Then the initial message $\left(q_{0_{i}}\right)$ passed from the bit node to the check node is [33]:

$$
q_{0_{i}}=\log \frac{P\left(X_{i}=1 \mid Y_{i}, A_{i}\right)}{P\left(X_{i}=-1 \mid Y_{i}, A_{i}\right)}=\frac{2}{\sigma_{N}^{2}} Y_{i} A_{i}
$$


For Rayleigh fading channel without SI (no SI is available to the decoder) we can approximate $q_{0_{i}}$ as given in [33]:

$$
q_{0_{i}} \approx \frac{2}{\sigma_{N}^{2}} Y_{i} \cdot E\left[A_{i}\right],
$$

where $E\left[A_{i}\right]=0.8862$.

\subsection{Performance of FEC
Rayleigh fading Channels}

In our simulations, we use standard $512 \times 512,8$ bit monochrome "Lena" image and model the noisy channel as Rayleigh flat-fading channel with additive white Gaussian noise. The information block length ( $k$ ) of the RC-LDPC codes is 384 bytes (3072 bits).

The mother code matrix is $1920 \times 4992$ (bits) and the extension matrix $\left(h_{\text {ext }}\right)$ is $384 \times 384$ (bits). The mother code matrix and extension matrix are constructed with PEG as shown in Fig. 4.3 with the following bit node degree distribution which is optimized by density evolution for rate 0.5 over uncorrelated Rayleigh fading channels with SI [33]:

$$
\lambda(x)=0.292439 x^{2}+0.253636 x^{3}+0.060454 x^{4}+0.03161 x^{9}+0.361861 x^{10}
$$

In (5.4) the powers of $x$ indicate the degrees of the bit nodes and the coefficients of $x$ show the percentages of bit nodes with those degrees.

Figure 5.2 (a) and (b) show the error-correction performance of RC-LDPC codes over uncorrelated Rayleigh fading channels. Figure 5.2 (a) gives the WER curves for the RCLDPC codes for uncorrelated Rayleigh channel with SI. The codeword lengths corre- 
sponding to the rates of $\{8 / 8,8 / 9.75,8 / 9.875,8 / 10,8 / 10.25,8 / 10.5,8 / 10.75,8 / 11,8 / 11.5$, $8 / 12,8 / 12.5,8 / 13,8 / 14,8 / 15,8 / 18$ and $8 / 22\}$ are $\{384,456,468,474,480,492,504$, $516,528,552,576,600,624,672,720,864$ and 1056$\}$ (bytes). The set of rates are chosen such that they are almost uniformly distributed over a wide range of SNR. In Figure 5.2 (b), the WER curves are given for uncorrelated Rayleigh fading channel without SI. We haven't used optimal symbol degree distributions for fading channels without SI instead we use the same degree distribution as in (5.4). As in the previous case (with SI), in total, eleven channel coding rates are available with the same information block length. By comparing Figure 5.2 (a) with Figure 5.2 (b), we observe that the error-correction performance with SI is generally better than that without SI. Due to the better knowledge of the channel parameter, the WERs of Figure 5.2 (a) are about $3 \mathrm{~dB}$ better than those given in Figure 5.2 (b). Accordingly, the end-to-end PSNRs of the fading channel with SI are expected to be better than those of the fading channel without SI.

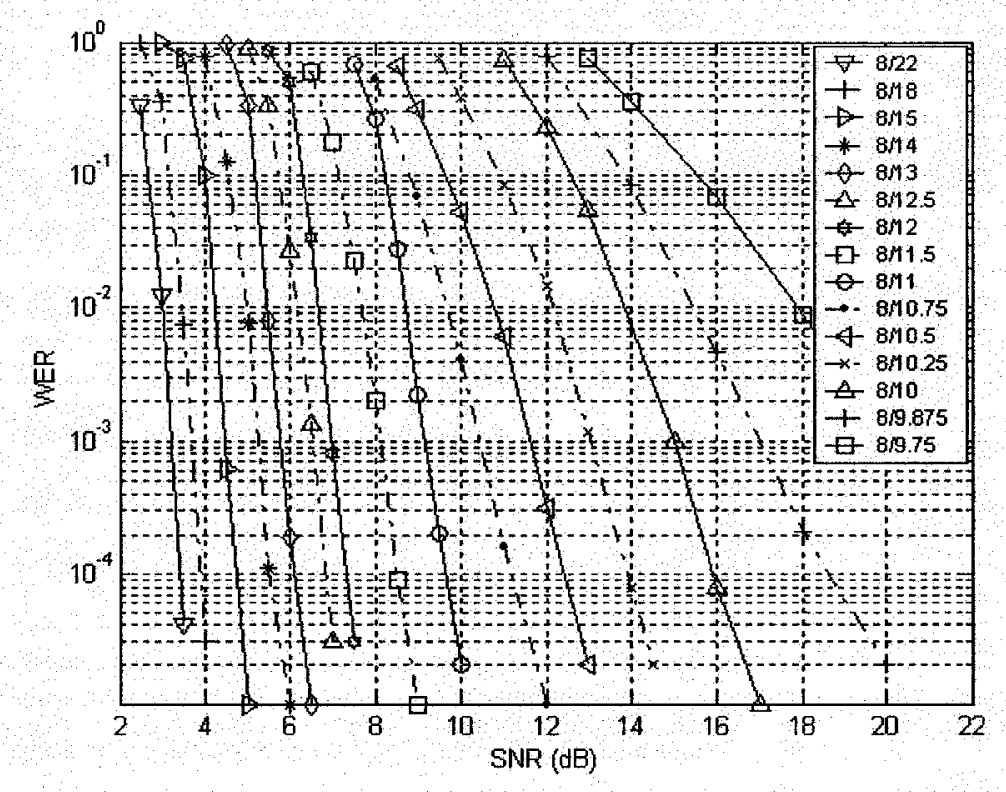

Figure 5.2 (a) Uncorrelated Rayleigh fading channel with SI. 


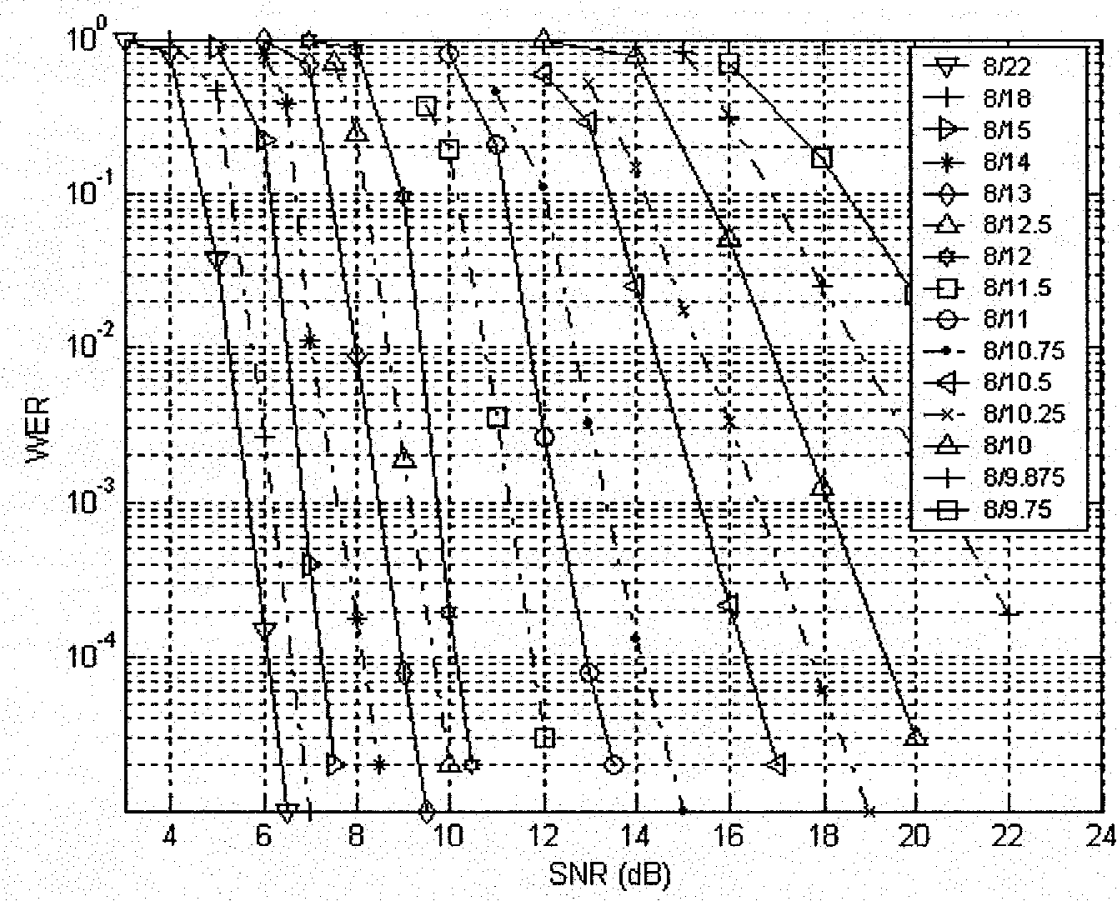

Figure 5.2 (b) Uncorrelated Rayleigh fading channel without SI.

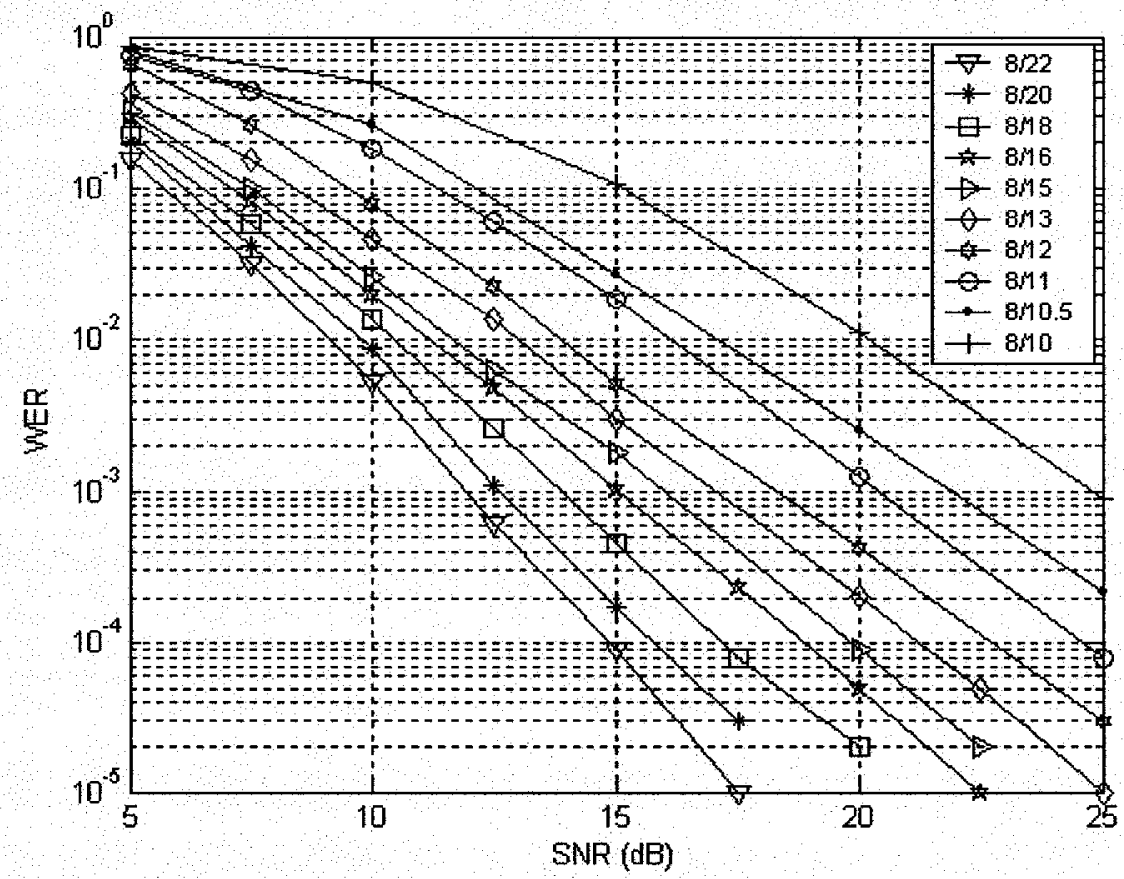

Figure 5.2 (c) Correlated Rayleigh fading channel; Vehicle speed $V=4 \mathrm{~m} / \mathrm{h}$. 


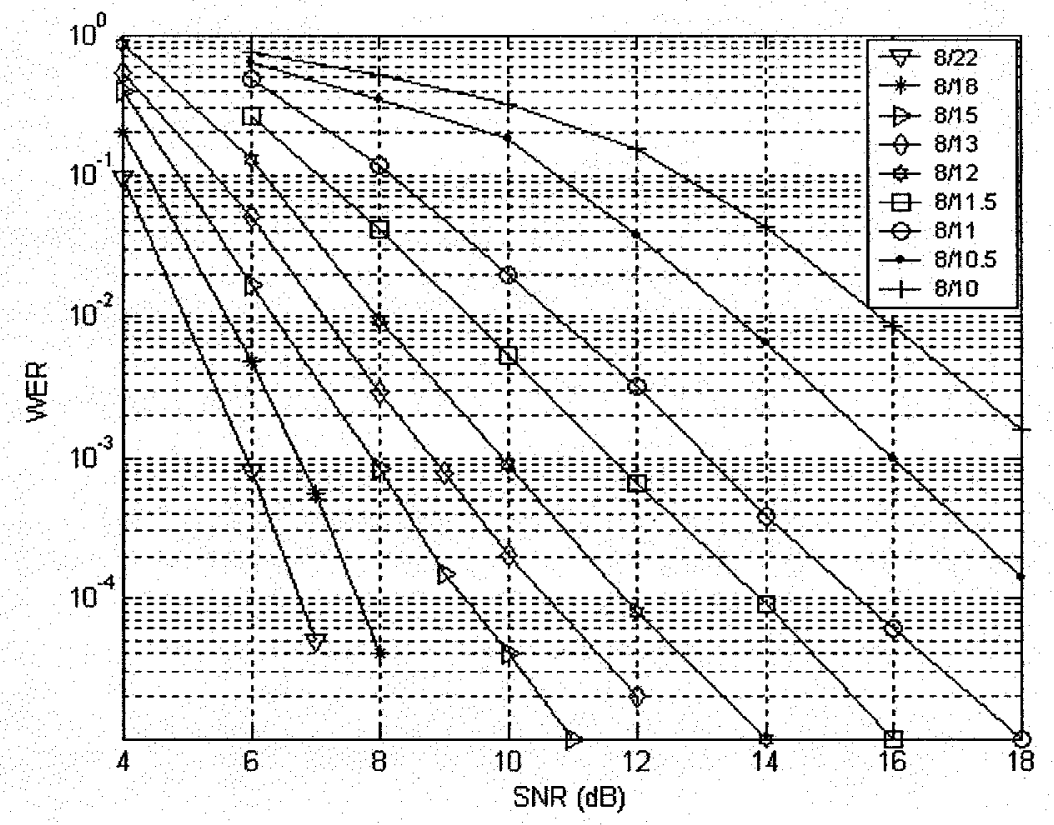

Figure 5.2 (d) Correlated Rayleigh fading channel; Vehicle speed V $=30 \mathrm{~m} / \mathrm{h}$.

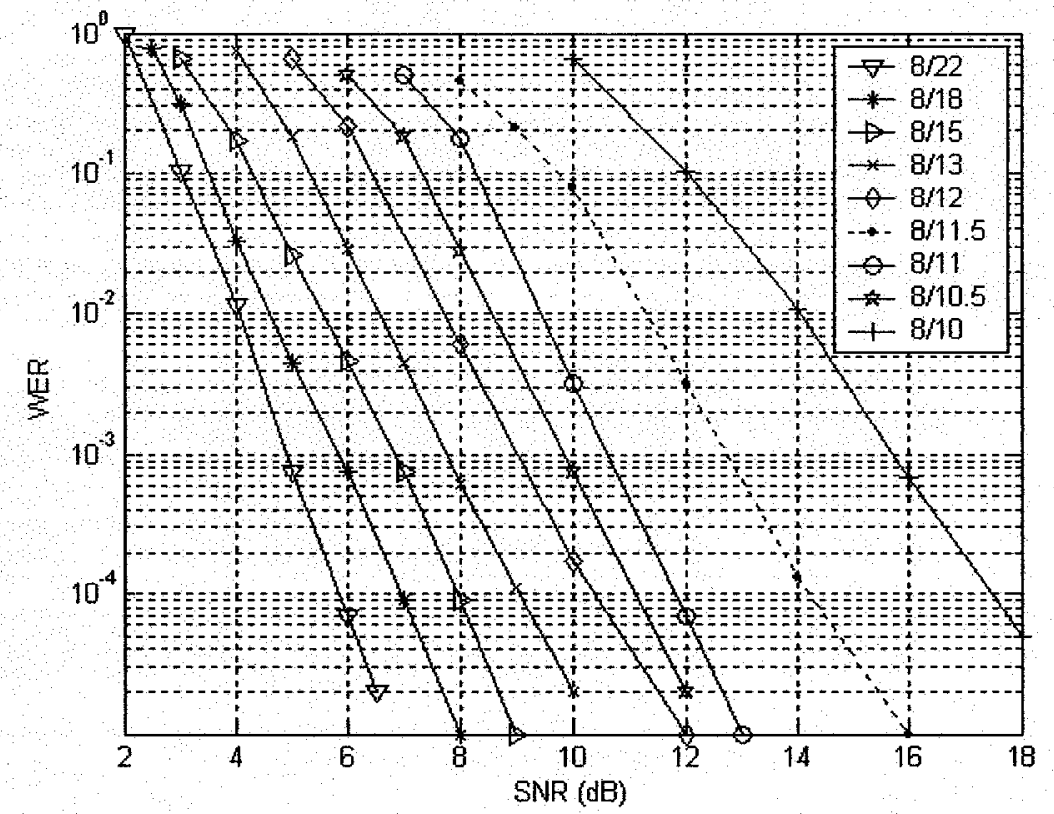

(e) Correlated Rayleigh fading channel; Vehicle speed $\mathrm{V}=70 \mathrm{~m} / \mathrm{h}$.

Figure 5.2: WER curves of RC-LDPC codes for different Rayleigh fading channels; $\mathrm{K}=$ 384 bytes 
We also show the WER curves for correlated Rayleigh fading channels in Fig 5.2 (c) (e). We also use the same degree distribution as (5.4) and the results are obtained assuming perfect SI. In this experiment, the correlated Rayleigh fading channels are simulated using Jakes' model [34]. The carrier frequency is set as $900 \mathrm{MHz}$ and the source rate is assumed to be $9.6 \mathrm{~kb} / \mathrm{s}$. The typical mobile speeds of $4 \mathrm{~m} / \mathrm{h}, 30 \mathrm{~m} / \mathrm{h}$ and $70 \mathrm{~m} / \mathrm{h}$ are tested. The corresponding normalized Doppler shifts $\left(f_{d}\right)$ are $1.85 \times 10^{-4}, 1.39 \times 10^{-4}$ and $3.24 \times 10^{-3}$ respectively. The channel codeword lengths for the speed of $4 \mathrm{~m} / \mathrm{h}$ are $\{480$, $504,528,576,624,720,768,864,960$ and 1056$\}$ (bytes), and are $\{480,504,528,552$, $576,624,720,864$, and 1056 \} (bytes) for $30 \mathrm{~m} / \mathrm{h}$ and $70 \mathrm{~m} / \mathrm{h}$. These sets of rates can be easily obtained by puncturing and extending the parity-check matrix of the mother code. By observing the three figures, we can find that when the speed of the vehicle increases, the error-correction performance of the codes tends to be better. As a result, the performance of the code approaches that of uncorrelated Rayleigh fading channel when the speed increases, because of the decrease in correlation among fading coefficients.

When the RC-LDPC codes given in Figure 4.3 are employed to transmit JPEG2000 encoded "Lena" image over Rayleigh fading channels, the end-to-end PSNRs are shown in Figure 5.3 (a)-(e). Figure 5.3 (a) shows the PSNRs for uncorrelated Rayleigh fading channels with SI. Also shown in the figure are the capacity values of PSNR, which is the upper bound of PSNRs for uncorrelated Rayleigh fading channel with SI. The capacity values are obtained by assuming that the source images are encoded at the source rates corresponding to the capacities $(C)$ [35] of the Rayleigh fading channel subject to the constraint of the total bit budget $(C \times B)$ and assuming error free conditions. To show the performance of the joint JPEG2000/RC-LDPC system, we also transmit "Lena" image 
with three different total bit budgets of $0.252 \mathrm{bpp}, 0.505 \mathrm{bpp}$ and $0.994 \mathrm{bpp}$. As can be seen from Figure 5.3 (a), the end-to-end PSNRs are low when the corresponding channel SNR is small. This is expected as high probability of error for channel codes. With the increase of channel SNR, the end-to-end PSNRs improve accordingly. The capacity values of the PSNR, as upper bounds, help us to evaluate the performance of our system. It can be shown from the figure that the end-to-end PSNRs of our scheme are generally close to the upper bound values especially at higher values of SNR. In particularly, when the transmission rate is $0.252 \mathrm{bpp}$, our joint JPEG2000/RC-LDPC system achieves the smallest distance to the capacity of $0.96 \mathrm{~dB}$, where the channel SNR is $8 \mathrm{~dB}$. We observe similar behaviors of PSNRs in Figure 5.3 (b), where the end-to-end PSNRs for uncorrelated Rayleigh fading channel without SI are illustrated. The capacity values [36] are also included in (b) for comparison. We can find in (b) that the smallest gap to the capacity is $0.7 \mathrm{~dB}$ at the SNR of $12 \mathrm{~dB}$ and transmission rate of $0.994 \mathrm{bpp}$.

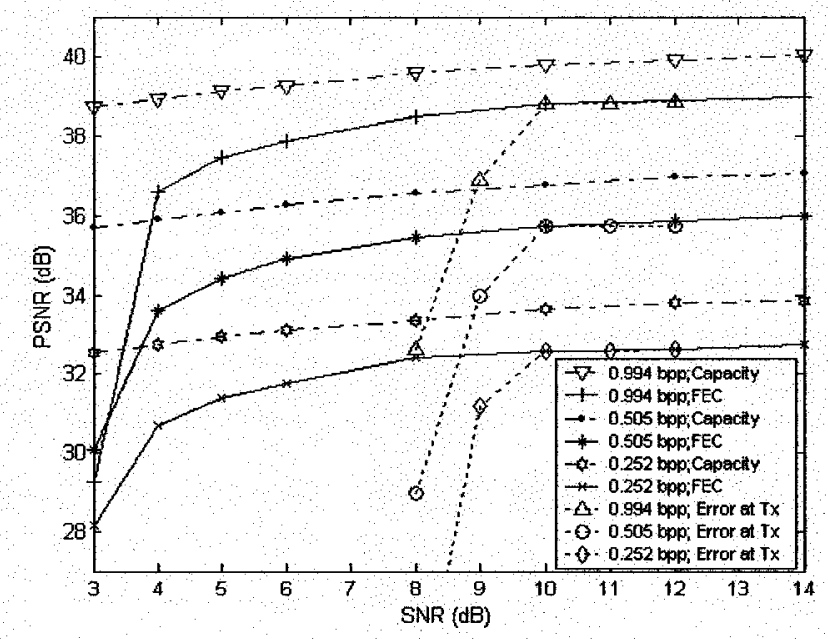

Figure 5.3 (a) PSNRs for uncorrelated Rayleigh fading channel with SI compared with capacity values; $S_{\text {estimate }}=10 \mathrm{~dB}$. 


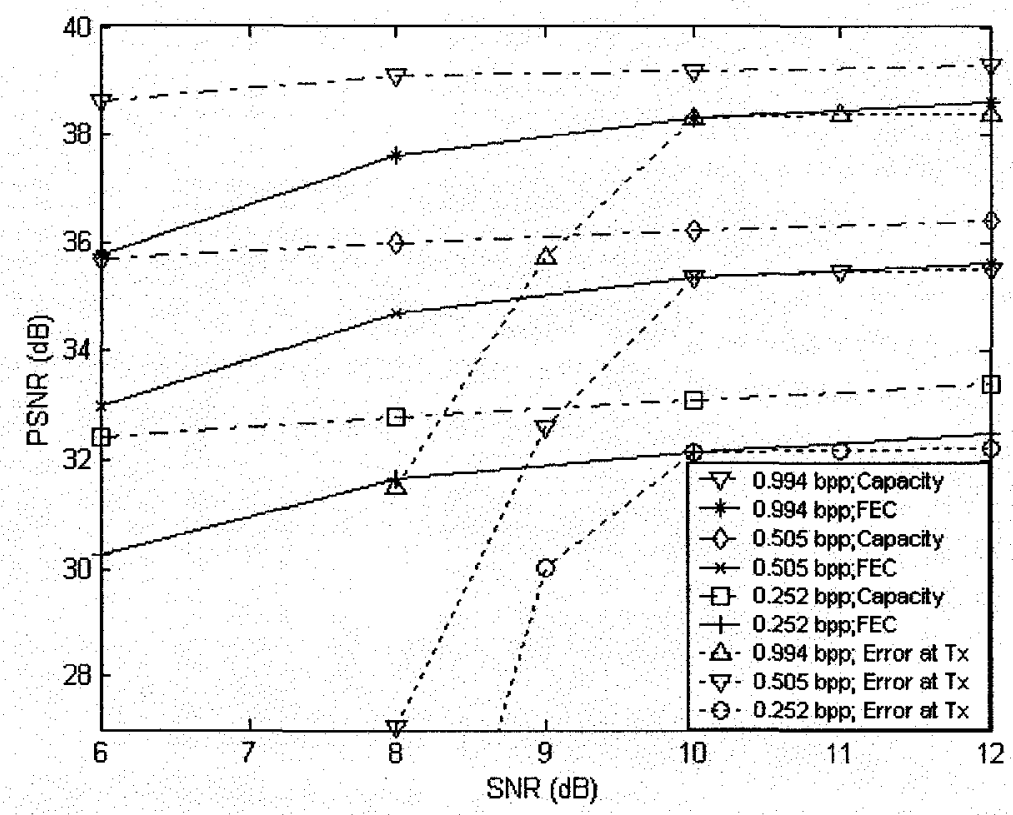

Figure 5.3 (b) PSNRs for uncorrelated Rayleigh fading channel without SI compared with capacity values; $S_{\text {estimate }}=10 \mathrm{~dB}$.

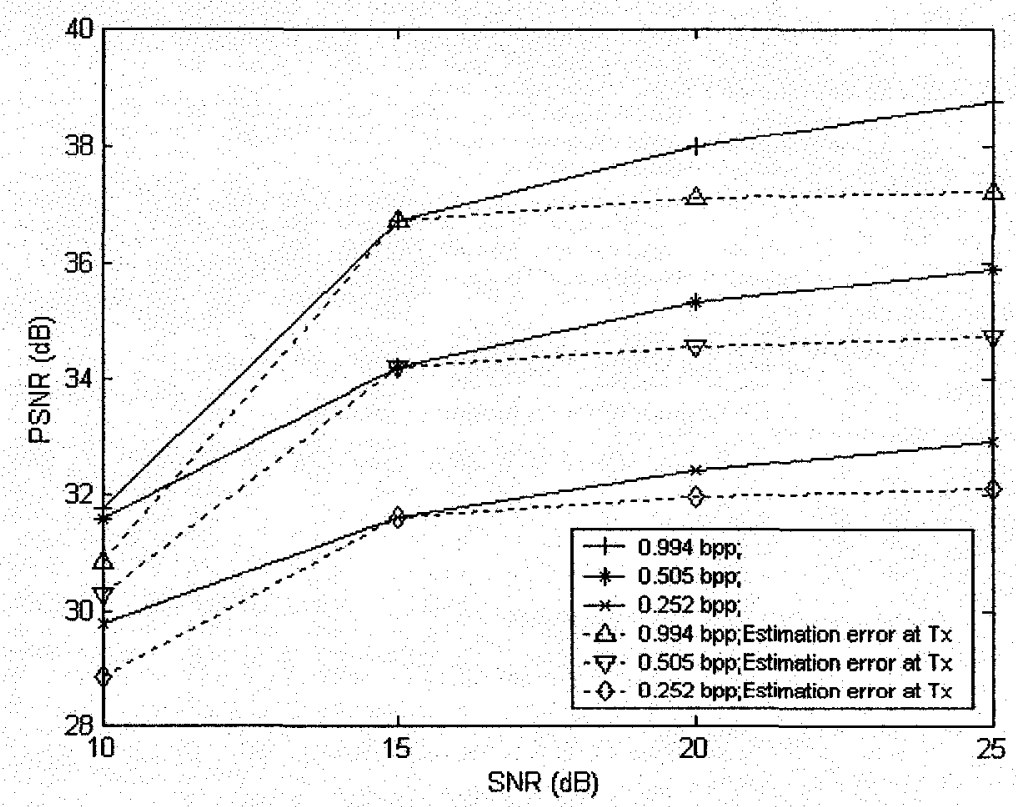

Figure 5.3 (c) PSNRs for correlated Rayleigh fading channel; Vehicle speed V $=4 \mathrm{~m} / \mathrm{h}$; $S_{\text {estimale }}=15 \mathrm{~dB}$. 


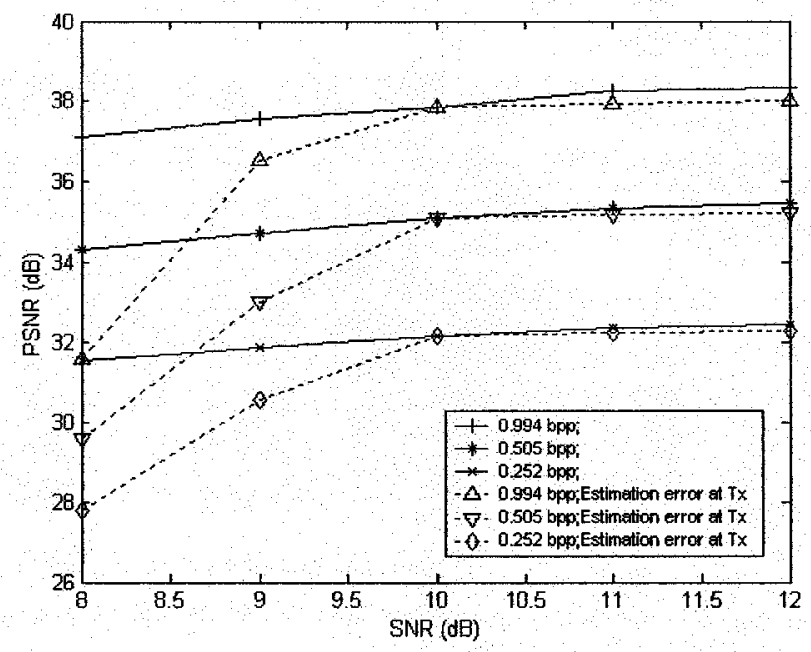

Figure 5.3 (d) PSNRs for correlated Rayleigh fading channel; Vehicle speed $V=30 \mathrm{~m} / \mathrm{h}$; $S_{\text {estimate }}=10 \mathrm{~dB}$.

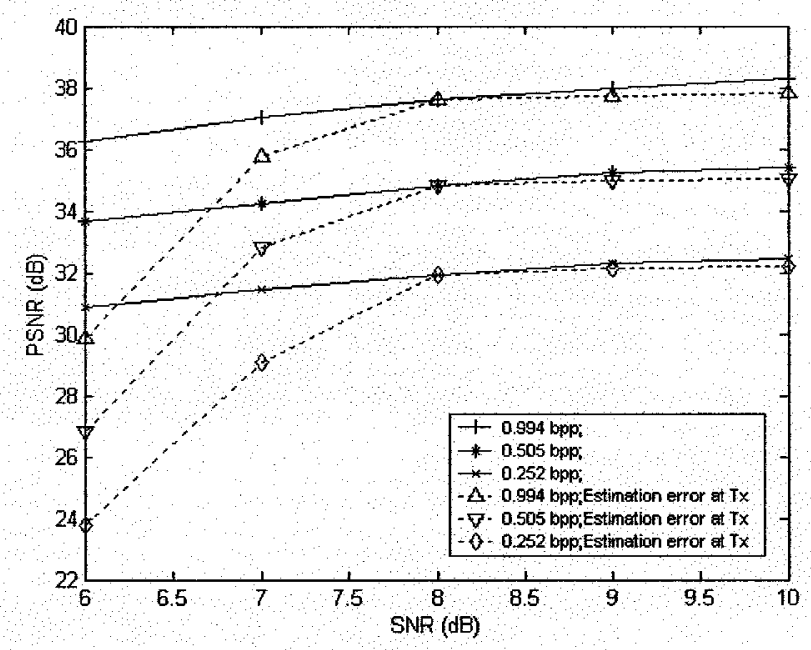

Figure 5.3 (e) PSNRs for correlated Rayleigh fading channel; Vehicle speed V=70 m/h; $S_{\text {estimate }}=8 \mathrm{~dB}$.

Figure 5.3: End-to-end PSNRs for JPEG2000 encoded 512×512, 8 bit monochrome "Lena" image over different Rayleigh fading channels; including the PSNRs for underestimation and over-estimation assuming the source image is encoded for the estimated channel SNR. 
Similarly, Figure 5.3 (c)-(e) shows the end-to-end PSNR for correlated Rayleigh fading channels. The vehicle speeds are $4 \mathrm{~m} / \mathrm{h}, 30 \mathrm{~m} / \mathrm{h}$ and $70 \mathrm{~m} / \mathrm{h}$ for (c), (d) and (e) respectively. When the speed increases, the correlation of the fading coefficients reduces accordingly. As a result, the codes' performance and PSNRs improve as well.

\subsection{Estimation Errors for Channel Parame-}

\section{ters}

In wireless communications, the channel is time-variant so that it is difficult to obtain the perfect knowledge of the channel conditions. In order to allocate proper channel code rates, channel SNR has to be perfectly known to the transmitter (Tx) in a FEC system. When channel SNR of the fading channel is perfectly known, the corresponding correct WERs for each channel code rate can be obtained. Thus, we can correctly obtain this set of input parameters to our rate-allocation algorithm. This ensures that our rate-allocation algorithm can allocate the rates optimally.

However, in practice, the transmitter uses estimated channel SNR for a FEC system. It may happen that the estimated channel SNR is different from the actual one. The errors in estimation of channel parameters will result in source images that are compressed with non-optimal compression ratios. In addition, the channel coding rates assigned to the packets are not optimal. Two opposite phenomena, under-estimation and over-estimation exist in our work. The effects of error estimation can be studied in the following two scenarios: 
In the first scenario, the channel parameter is under-estimated. Let us assume the source image is encoded based on an estimated channel parameter of $S N R_{\text {estimared }}$ and the actual channel parameter is $S N R_{\text {actual }}$. Under-estimation means that the estimated SNR is less than the actual one ( $\left.S N R_{\text {estimated }}<S N R_{\text {actual }}\right)$. The channel coding rates are allocated assuming a worse channel condition. The WER for each channel coding rate is less than the correct ones (true optimal based of actual SNR); we have a smaller $P_{\mathrm{i}}$ in (2.1). Consequently, the expected distortion may be reduced, resulting in a better PSNR than the estimated one. However, from the rate-allocation algorithm, we know that the system is still not optimal. The reason is that the system is sending too much redundancy (more than needed) and the performance is going to drop as it hasn't used enough bits to represent the source. The results of under-estimation of channel condition on the transmission of encoded images are also shown in Fig. 5.3.

In the second scenario, the estimated SNR is larger than the actual one $\left(S N R_{\text {estimated }}>S N R_{\text {actual }}\right)$, which we will refer to as over-estimation. Contrary to the first scenario, the channel coding rates are allocated assuming a better channel condition. The WER for each channel coding rate is greater than the correct ones. We then have a bigger $P_{\mathrm{i}}$ in (2.1). As a result, the expected distortion will increase, resulting in a worse PSNR than the estimated one. We also know, from the rate-allocation algorithm, that the system is far from optimal. The system represents the source with too many bits and it has too little redundancy to protect them. The system basically transmits everything but as the protection is not enough with very high probability earlier packets will have errors and then at the decoder a large portion of the bitstream will be lost. Compared with the previous scenario where the channel condition is under-estimated, the truncation has severe 
influence on the end-to-end PSNRs. Practically, we need transmission systems, even when ideal channel parameters are not available at the transmitter. The influence of error in estimating channel SNR at the transmitter on the PSNRs can be seen in Figure 5.3 (a) (e). In Figure 5.3 (a), the estimated SNR is $10 \mathrm{~dB}\left(S N R_{\text {estimated }}=10 \mathrm{~dB}\right)$. The optimal rateallocation algorithm is applied according to this channel parameter. When underestimation occurs $\left(S N R_{\text {actual }}>10 \mathrm{~dB}\right)$, the PSNRs only increase slightly. This scenario is depicted in the figure with part of the dashed curves to the right of $10 \mathrm{~dB}$. We can see that they are slightly better than the PSNR for the SNR of $10 \mathrm{~dB}$. They are, however, still worse than the PSNRs corresponding to no estimation error for the same SNRs. This is of course because the latter PSNRs are the optimal values for such channel conditions. Furthermore, we can find that there are sharp drops for the dashed curves to the left of $10 \mathrm{~dB}$. This corresponds to the over-estimation case, where the actual SNR is less than the estimated one $\left(S N R_{\text {actual }}<10 \mathrm{~dB}\right)$. To explain this, we know from Figure 5.2 (a) that when SNR is decreased below $10 \mathrm{~dB}$, the WERs of channel codes that are used to protect the packets will decrease sharply. Consequently corresponding PSNR will have a sharp drop and will be far from optimal. To check the effect of estimation-error at transmitter, the simulations are also performed for uncorrelated Rayleigh fading channel without SI for the estimated SNR of $10 \mathrm{~dB}$. The results are shown in Figure 5.3 (b) by dashed lines. We observe similar tendencies as in uncorrelated Rayleigh fading channel with SI.

In Figure 5.3 (c) - (e), we observe the effect of estimation-error on correlated Rayleigh fading channels. As can be seen, they follow similar trends as the uncorrelated cases. By comparing the dashed curves in (a) to (e), we also observe that the system is more tolerant to the estimation error at the transmitter when the speed of the mobile is low. This is 
because the rate-allocation algorithm is related to the choice of channel coding rates. If the error correction curves drop sharply with the channel parameter, a little offset of channel parameter will lead to the dramatic change of WER. We know that the WER for low-speed mobile does not change as sharply as that of high-speed mobile. So the system is more tolerant to the estimation error at the transmitter when the speed of the mobile is low

With the results of under-estimation and over-estimation in Figure 5.3 (a) to (e), we find out that the system is much sensitive to over-estimation and relatively robust to under-estimation. In order to improve the robustness of our system, we could make our system a little under-estimated. At the transmitter, we can apply our rate-allocation algorithm according to the channel SNR that is slightly worse than the actual one. Then from our simulation results, we know that, although the results are not optimal, we could still achieve rather good end-to-end PSNRs. These results suggest that a biased decision towards underestimating the channel SNR at the transmitter can be beneficial

To show how our combined source and channel coding system with RC-LDPC codes improve over the existing JSCC schemes for fading channels under the same circumstances, we consider transmission of standard $512 \times 512,8$ bit monochrome "Lena" image over correlated Rayleigh flat-fading channels at the total transmission rate of 0.25 bpp. The image is encoded with SPIHT coder. In Figure 5.4, we show the PSNRs against different normalized Doppler spreads from $1 \times 10^{-5}$ to $2 \times 10^{-4}$. The schemes are optimized for the SNR of $13 \mathrm{~dB}$ and Doppler spread of $5 \times 10^{-5}$. From Figure 5.4, it can be seen that the PSNRs of combined SPIHT/RC-LDPC system are generally better than those of [5] and [6] over a wide range of normalized Doppler spread. The improvement over [5] in 
PSNR is as large as $1.3 \mathrm{~dB}$, when $f_{\mathrm{d}}$ equals $2 \times 10^{-4}$. The improvement over [6] in PSNR is up to about $3.6 \mathrm{~dB}$, when $f_{\mathrm{d}}$ equals $1 \times 10^{-5}$.

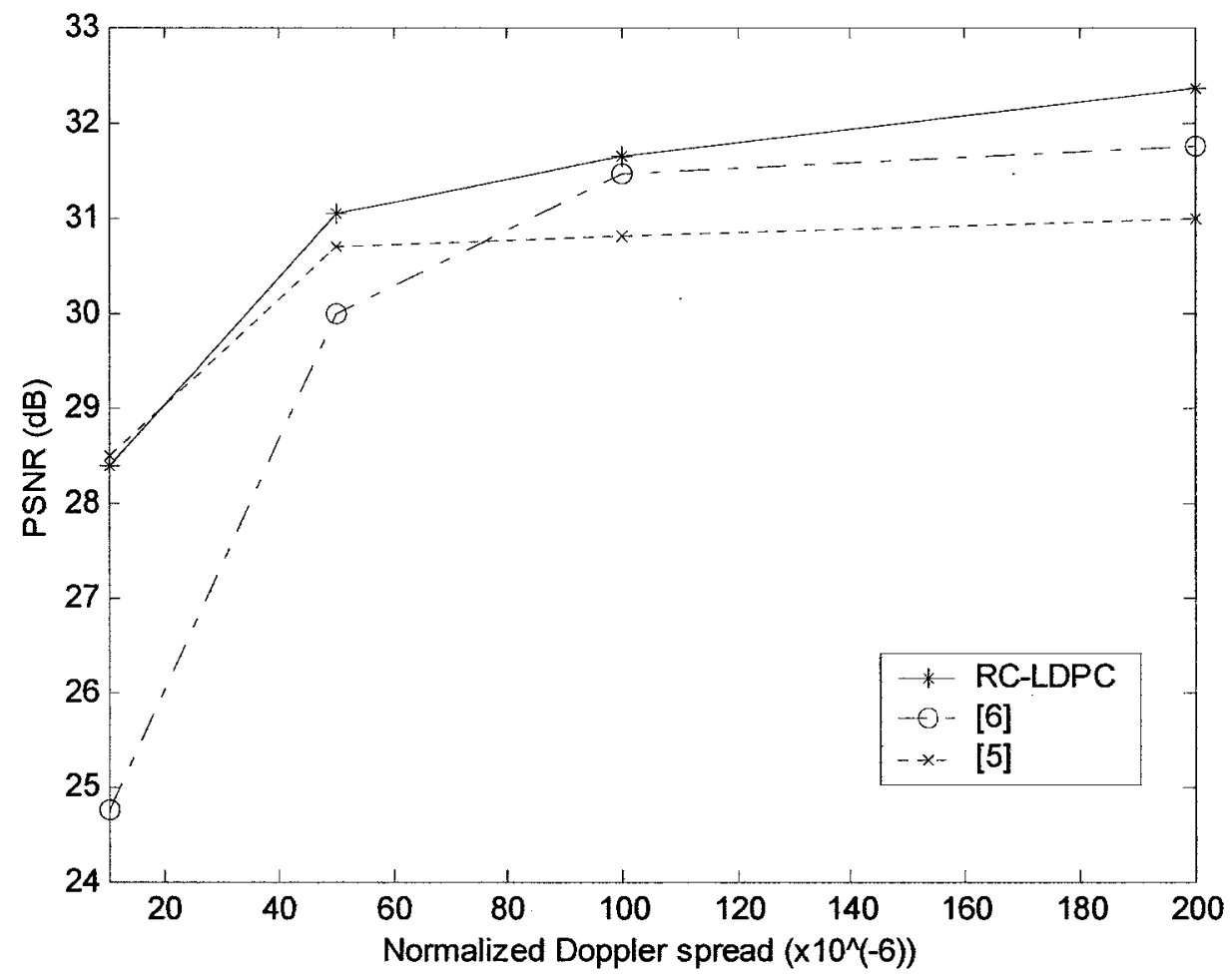

Figure 5.4: End-to-end PSNRs for SPIHT encoded "Lena" image for correlated Rayleigh fading channels with different normalized Doppler spread from $1 \times 10^{-5}$ to $2 \times 10^{-4}(\mathrm{SNR}=13 \mathrm{~dB}$, total transmission rate $=0.25 \mathrm{bpp})$. 


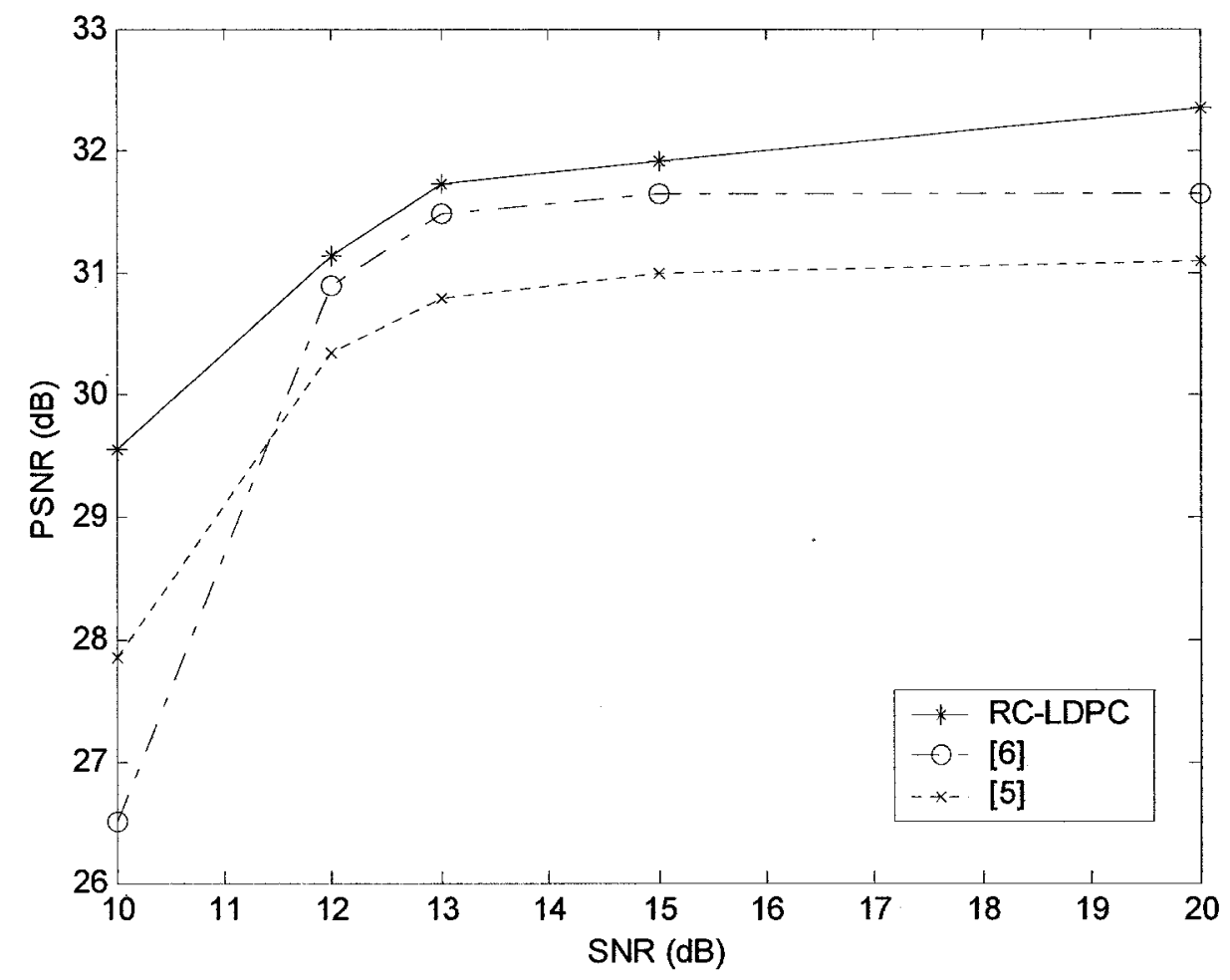

Figure 5.5: End-to-end PSNRs for SPIHT encoded "Lena" image for correlated Rayleigh fading channels with normalized Doppler spread of $1 \times 10^{-4}$ ( the total transmission rate is $0.25 \mathrm{bpp}$ )

To further illustrate the improvement over [5] and [6], the image is transmitted over the Rayleigh fading channel with $f_{\mathrm{d}}$ of $1 \times 10^{-4}$. The PSNRs are shown in Figure 5.5 for actual SNRs. The schemes are optimized for the SNR of $13 \mathrm{~dB}$ and Doppler spread of $10^{-4}$. The improvements are significant, especially for the SNR of $10 \mathrm{~dB}$, where our system achieves up to about $1.7 \mathrm{~dB}$ and about $3 \mathrm{~dB}$ improvements over [5] and [6] respectively.

We have also tested standard "Goldhill" and "Barbara" images over the above uncorrelated or correlated Rayleigh fading channels with or without SI. Both combined 
JPEG2000/RC-LDPC coder and SPIHT/RC-LDPC coder are employed. We obtained similar results as those of standard "Lena" image. 


\section{Chapter 6}

\section{Progressive Image Transmission}

\section{over Noisy Channels with ARQ}

\subsection{Introduction}

If a feedback channel is available, one can transmit data using ARQ schemes with a performance generally better than that of FEC. Using ARQ scheme however introduces more delay that may not be acceptable for some real-time applications. In this work, we consider applications where delay is not a major concern and focus on type-II hybridARQ system which has been shown to have a very good throughput.

For the fading channels with feedback, Nosratinia et al. [15] approximates the JSCC optimization by maximizing the throughput of the ARQ system. Due to the progressive nature of the source encoded bitstream, minimizing the distortion is approximately equivalent to maximizing the throughput of the system. In [15], the fading channel is designed using Gilbert-Elliott model. We focus on throughput as the performance measure. In this work, we also maximize the throughput but consider Rayleigh flat fading models for the channel. 


\subsection{System Analysis}

Figure 6.1 illustrates the block diagram of the ARQ system. A noiseless feedback channel is added to the previous FEC system and we remove the rate-allocation part from it. Similar to the FEC scheme, the source images are first progressively encoded. Then, the resulting bitstream is encoded with the LDPC codes at the highest rate. The channel coded packets are transmitted over the noisy channel. If an error happens to that packet, a NAK message is sent back to the RC-LDPC encoder from the feedback channel. The additional bits corresponding to the next higher rate channel code rate is sent. The successive incremental transmission of bits continues until the packet is correctly decoded. When even the lowest rate can't protect the packet, the original packet is re-sent. Finally, the recovered bitstreams are decoded by JPEG2000 decoder.

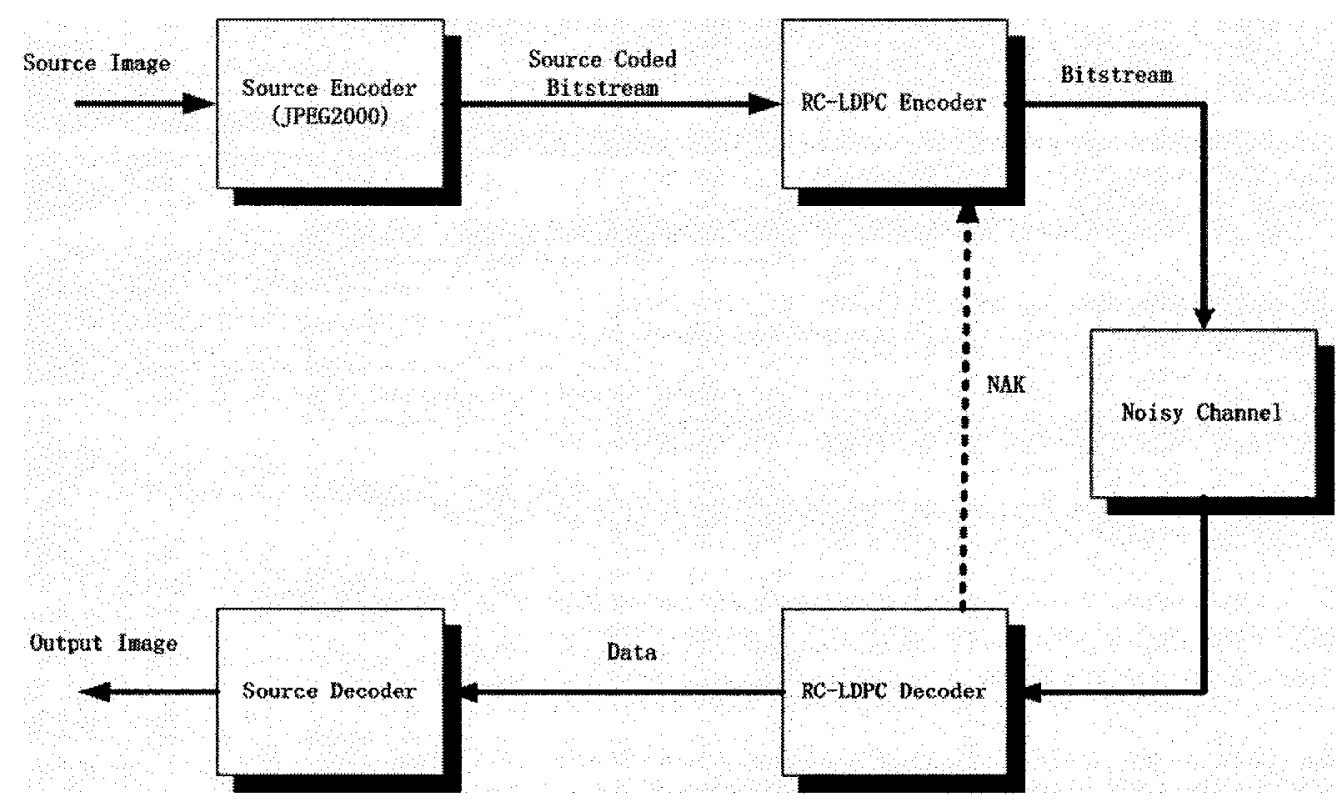

Figure 6.1: Block Diagram of ARQ Scheme. 
It has been shown in [1] that RC-LDPC codes used in a type-II hybrid-ARQ scheme over AWGN channel can achieve throughputs very close to the capacity at relatively short block lengths. In a type-II hybrid-ARQ System, the retransmission occurs automatically if all the parity bits are sent and yet a repeat-request signal (NAK) is received. When all the parity bits are sent if still a NAK is received then the transmitter has different options including retransmitting the information part of the packet.

The throughput $(\eta)$ for type-II hybrid-ARQ with RC-LDPC codes is [1]:

$$
\eta=\frac{K}{N_{1}\left(1-P_{1}\right)+\sum_{i=2}^{+\infty} N_{i}\left(1-P_{i}\right) \prod_{j=0}^{i-1} P_{j}}
$$

where $K$ is the information block length; $N_{i}$ is the length of the $i$ th packet; $P_{i}$ is the word WER of the $i$ th channel codes.

The assumption in the ARQ system is that all bits are eventually received correctly. So the throughput in fact shows the average number of bits that is received correctly at the receiver for each transmitted bit. As the throughput shows the ratios between the source and the total bits, one can obtain the source bit budget $(S)$ from the total bit budget $(B)$ using the following formula:

$$
S=B \times \eta,
$$

Since the source image is progressively encoded, and also due to the nature of the ARQ scheme, no rate allocation is required in this scheme. The optimal strategy is to transmit the source packets in the order they appear in the bitstream as long as the bit budget permits. 
The delay in type-II hybrid ARQ scheme is composed of two parts. The first part is the time used for transmission of the information packet at the first attempt and the incremental parity check bits at the subsequent attempts $\left(T_{t}\right)$ and NAK message $\left(T_{N A K}\right)$. The other part $T_{d}$ is the time used for decoding of the packet. Let us define $T_{u n i t}$ as the unit time by $T_{u n i t}=T_{t}+T_{N A K}+T_{d}$. The average delay $\left(T_{d e l a y}\right)$ of the scheme can be approximated by the following formula:

$$
T_{\text {delay }}=T_{t}\left(1-P_{1}\right)+\sum_{i=2}^{+\infty} i T_{\text {unit }}\left(1-P_{i}\right) \prod_{j=0}^{i-1} P_{j}
$$

where $P_{i}$ is the WER of the $i$ th channel codes.

In general, as can be seen from (6.3), delay is a function of the channel codes, channel parameter and the speed of transmission and processing.

\subsection{Performance of ARQ Scheme over BSC}

Using the equation (6.1) and based on the error-correction performance of RC-LDPC codes given in Figure 4.4, we can obtain the throughput of type-II hybrid-ARQ scheme as shown in Figure 6.2. It is obvious that the throughput decreases with the increase of $\varepsilon$. The dotted curve in Figure 6.2 represents the capacity of the BSC. 


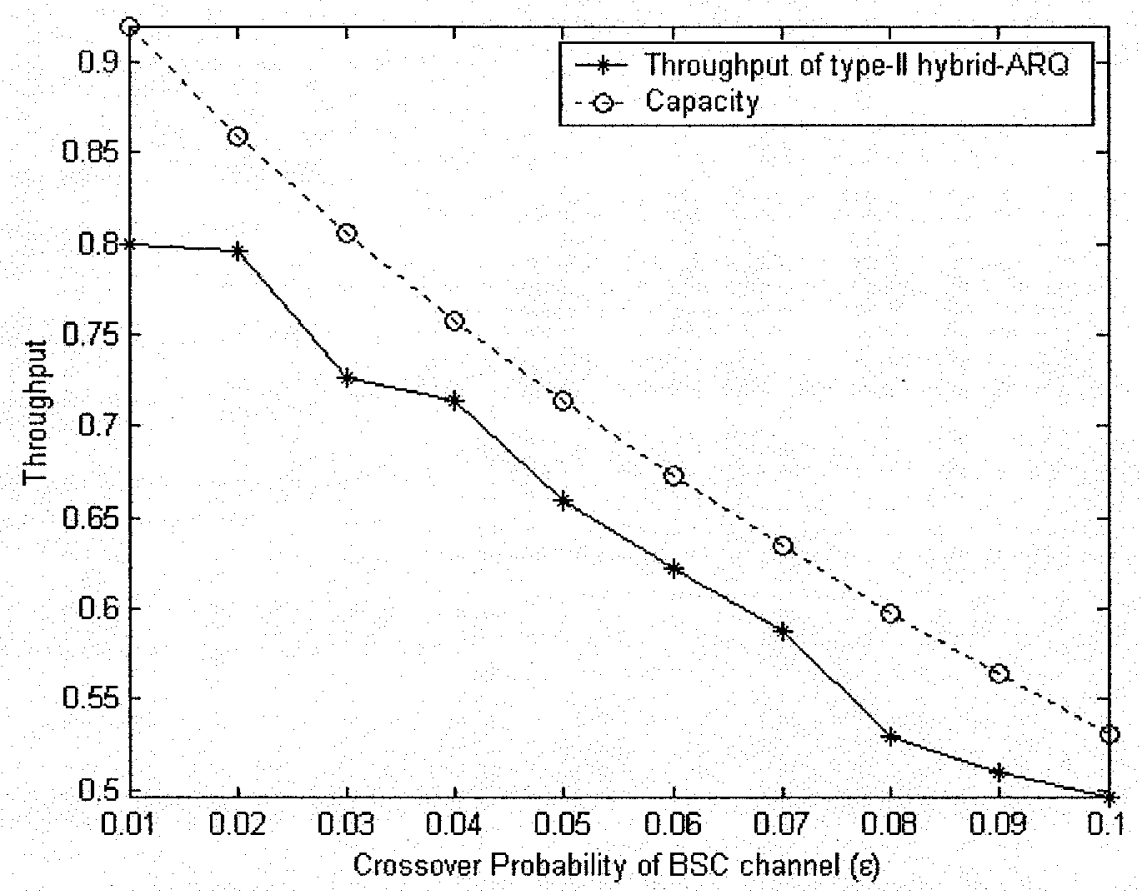

Figure 6.2: The throughput for type-II hybrid-ARQ scheme compared to the channel capacity $(\mathrm{K}=384$ bytes $)$.

We consider the transmission of standard $512 \times 512,8$ bit monochrome "Lena", and "Goldhill" images over BSC. We use the same set of RC-LDPC codes as in Chapter 4.

The information block length $\mathrm{K}$ is now 384 bytes and all the bits are used for coding the $\mathrm{J} 2 \mathrm{~K}$ bitstream. We have used (6.1) to obtain the source bit budget and then used that to obtain the PSNR at the output of JPEG2000 decoder assuming that the same number of bits are generated at the encoder and correctly received at the decoder. Figure 6.3 (a) and (b) show the expected PSNR of "Lena" and "Goldhill" respectively. For comparison, the results of the FEC scheme and error-free transmission at capacity are also given in Figures 6.3. We can find out the PSNR of the recovered images decreases when $\varepsilon$ increases. 
So the quality of the recovered image becomes worse with the increase of $\varepsilon$. Simulation results in Figure 6.3 show that the type-II hybrid-ARQ scheme can further improve the PSNR of the recovered image over FEC scheme. The difference between the ARQ scheme and the channel capacity results is generally less than $1 \mathrm{~dB}$, which means type-II hybrid-ARQ scheme with RC-LDPC codes can achieve near-capacity PSNRs.

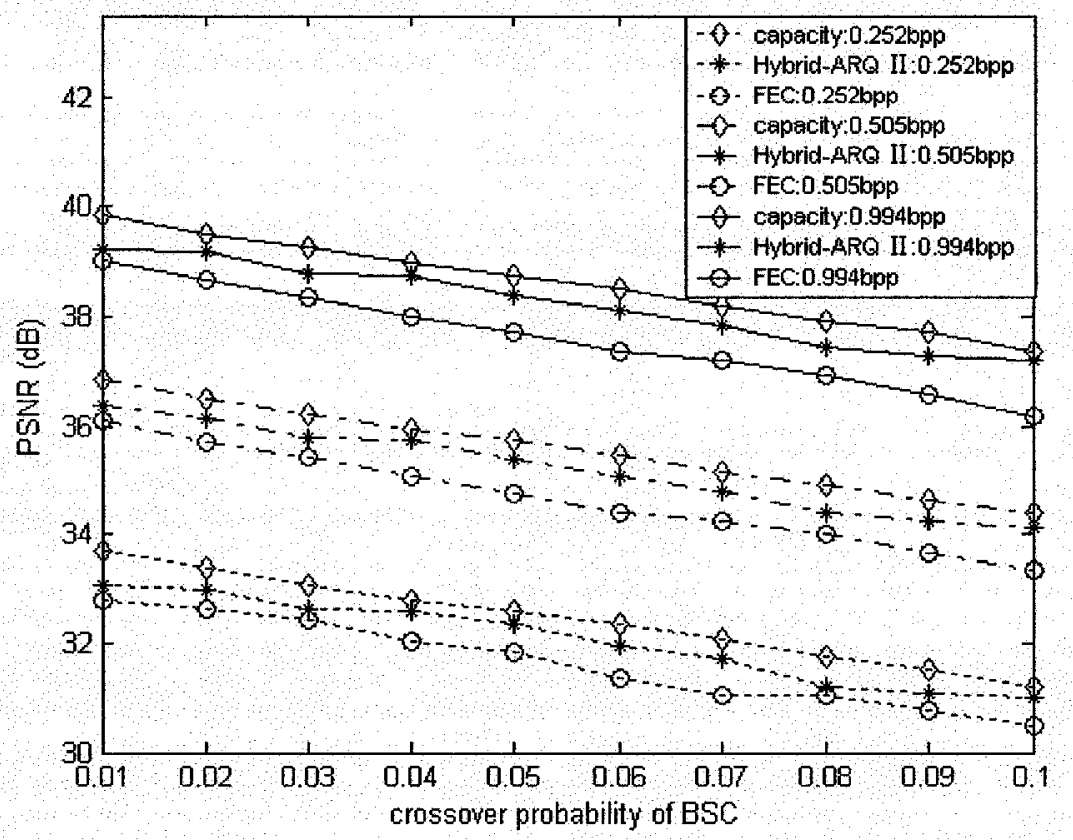

Figure 6.3 (a) "Lena" 


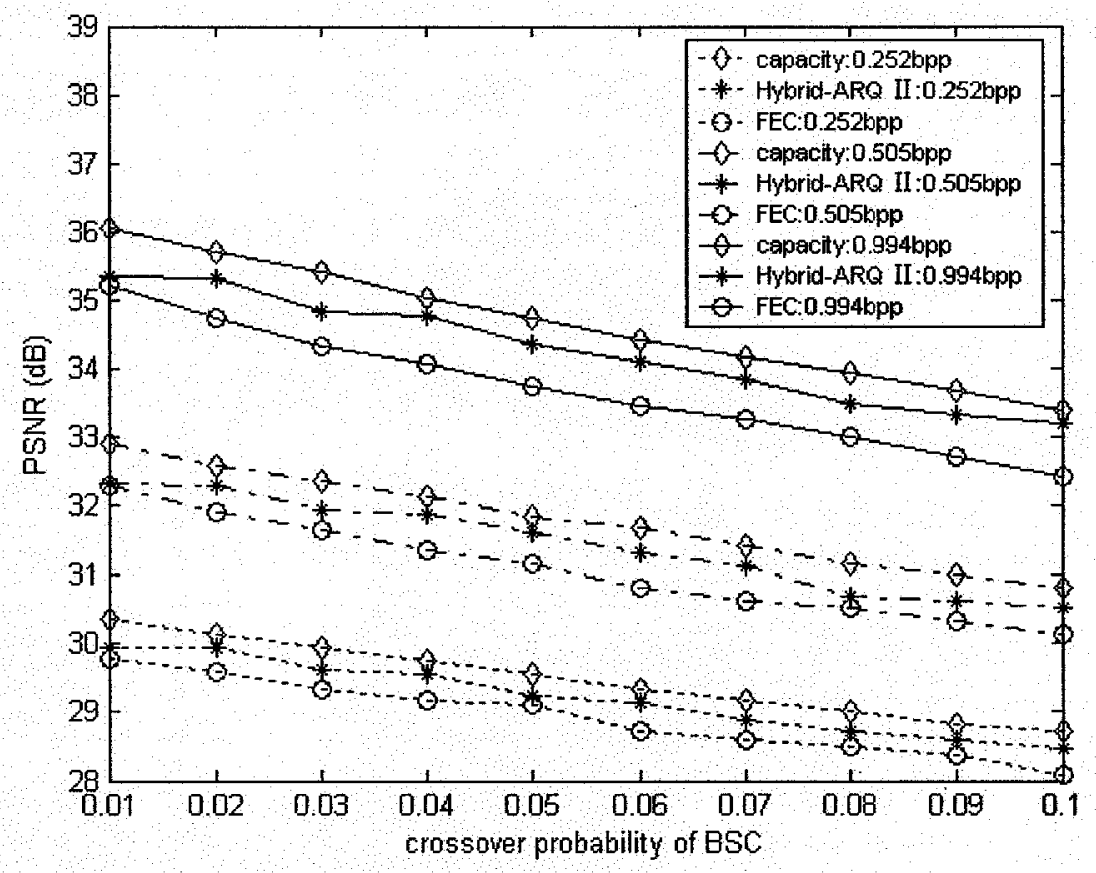

(b) "Goldhill"

Figure 6.3: Performance of FEC and hybrid-ARQ schemes ( $\mathrm{K}=384$ bytes)

\subsection{Performance of ARQ Scheme over}

\section{Rayleigh Fading Channels}

In our simulations, we use standard $512 \times 512,8$ bit monochrome "Lena", "Goldhill" and "Barbara" images over Rayleigh flat-fading channels. The images are source encoded using JPEG2000 algorithm. We use the same set of RC-LDPC codes as in Chapter 5 for uncorrelated/correlated Rayleigh fading channels with/without SI. 


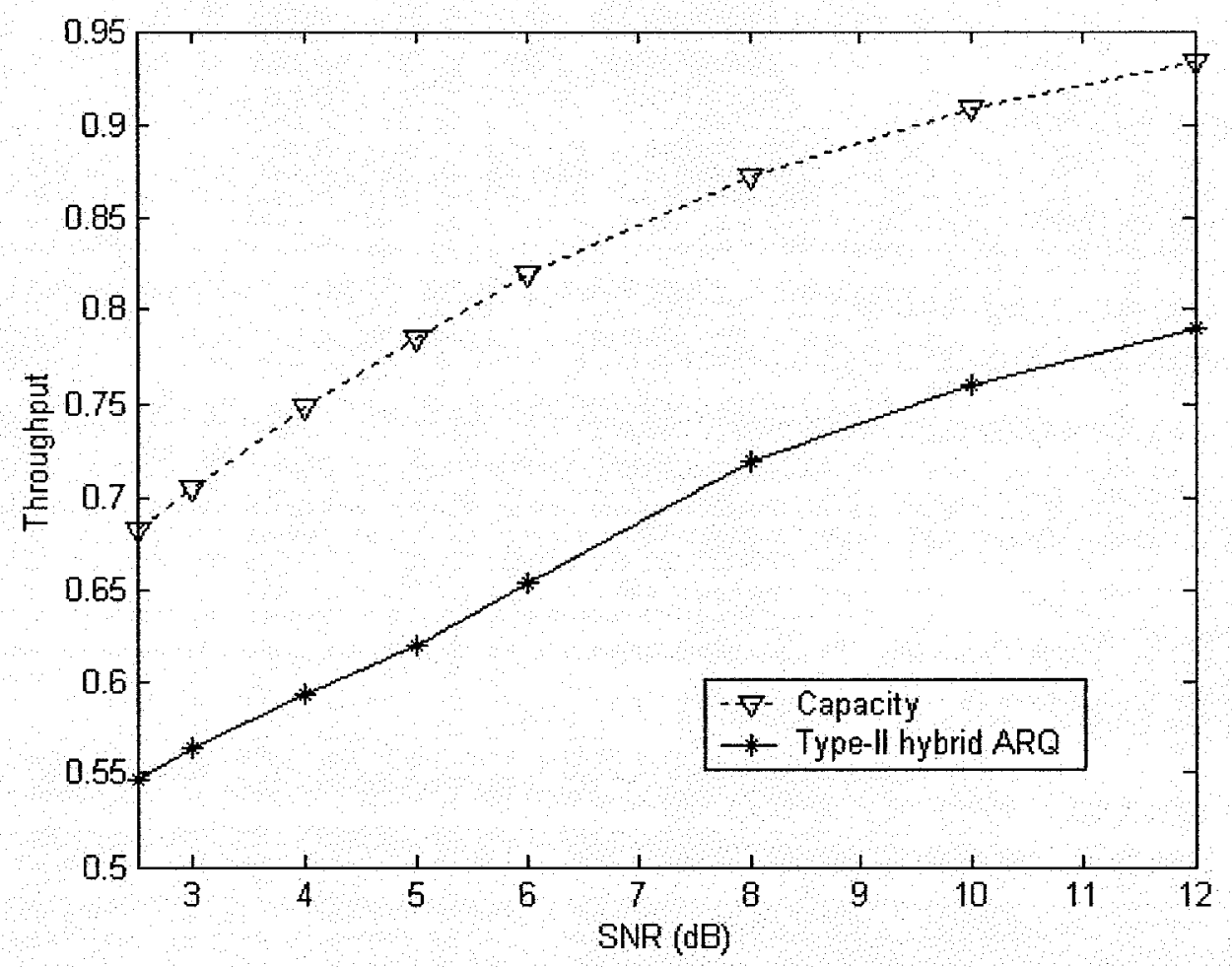

Figure 6.4: Throughput of Rayleigh flat-fading channel with SI, compared with channel capacity.

The throughput of the RC-LDPC codes over uncorrelated Rayleigh flat-fading channel is given in Figure 6.4. It shows the throughput of the RC-LDPC codes compared to the capacity of the uncorrelated Rayleigh fading channel with SI. The capacity of the uncorrelated Rayleigh fading channel is obtained from [35], which represents the upper bound on the throughput. We observe that by employing our codes, the distance between them can be reduced by up to 0.134 , where SNR equals $10 \mathrm{~dB}$. 


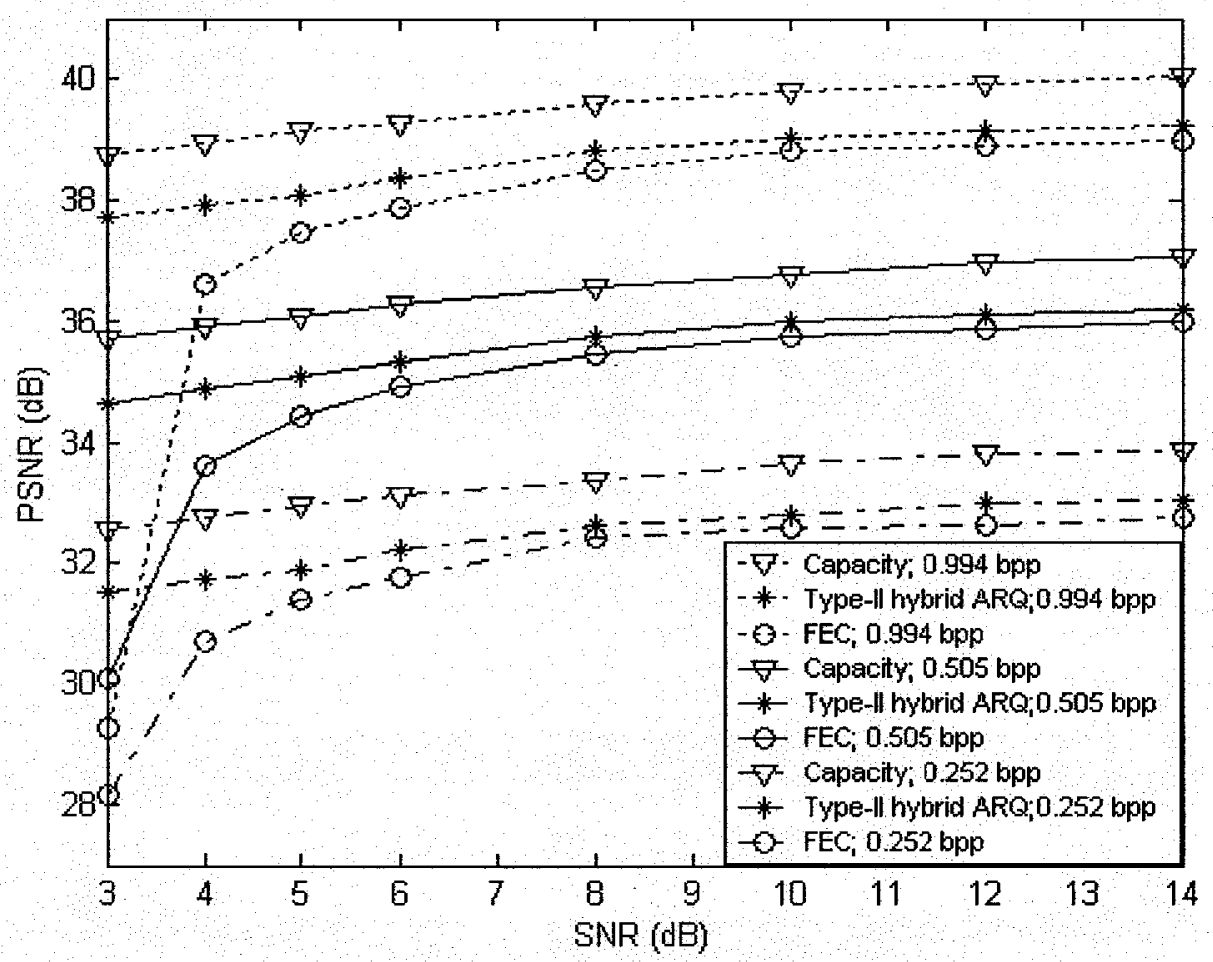

Figure 6.5: The PSNRs of FEC and ARQ schemes for uncorrelated Rayleigh flat-fading channel with SI compared with channel capacity values.

Figure 6.5 includes the PSNR curves of "Lena" for both the FEC and the type-II hybrid-ARQ systems. For the upper bound of PSNRs, the capacity curves are provided by transmitting the source encoded images at the channel capacity rates (capacity $\times$ total bit budget) and assuming an error free channel. This is done to illustrate how the PSNR will change with an increase in the total bit budget. Three total bit budgets of $0.252 \mathrm{bpp}, 0.505$ bpp and 0.994 bpp are employed. From Figure 6.5, we know that type-II hybrid-ARQ system can reduce the gap to capacity up to $0.75 \mathrm{~dB}$ for "Lena" at the SNR of $8 \mathrm{~dB}$ with the total rate of $0.252 \mathrm{bpp}$. We also find that when the SNR is low, corresponding to high probability of error, the PSNRs of type-II hybrid-ARQ System are much better than those 
of the FEC based system. With the increase of SNR, the gaps between the two systems become smaller.

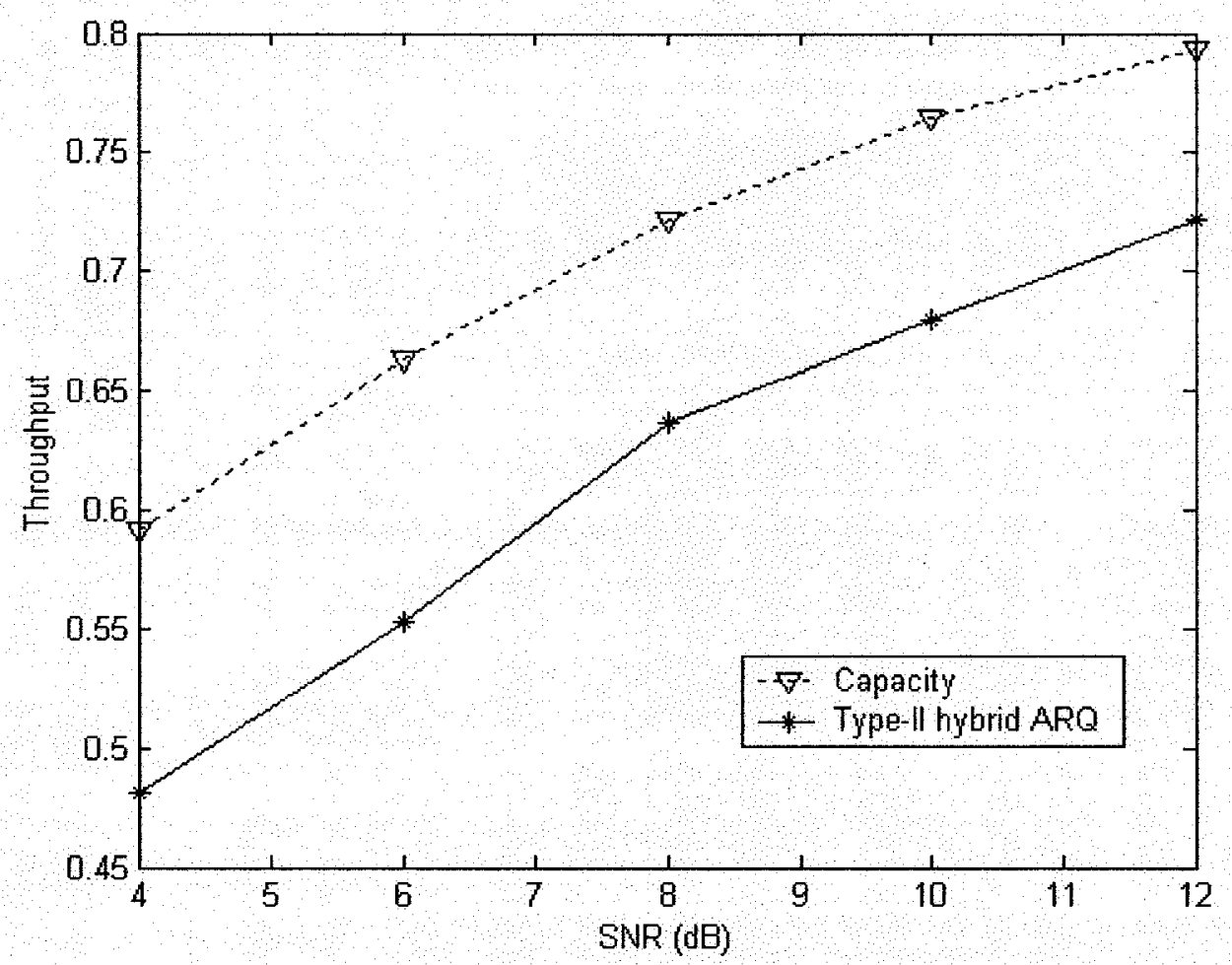

Figure 6.6: Throughput of Rayleigh flat-fading channel without SI compared with channel capacity.

Figure 6.6 shows the throughput for type-II hybrid-ARQ scheme with RC-LDPC codes over uncorrelated Raleigh fading channel without SI. This condition is discussed in Chapter 5. Since ideal side information is not known, the throughput is less than that of the case with SI. From Figure 6.6, we can see that the throughput for uncorrelated Rayleigh fading channel without SI is generally within the distance of about 0.09 from the capacity values (obtained from [36]). The smallest gap to capacity is 0.071 for the SNR of $12 \mathrm{~dB}$. 
With the RC-LDPC codes, we obtain the PSNR curves given in Fig 6.7. The PSNR curves in Figure 6.7 follow the same trend as those in Figure 6.5. Comparing Figure 56.6 with Figure 6.8, we can clearly see that the PNSRs in Figure 6.5 are generally better than those of Figure 6.7. This is expected as the system with ideal SI provides more information to the decoder, which helps to improve the coding performance. The smallest gap to the capacity is $0.4 \mathrm{~dB}$ for type-II hybrid-ARQ scheme when transmitting "Lena" at the SNR of $12 \mathrm{~dB}$ with the total rate of $0.252 \mathrm{bpp}$. The gap to capacity and the gap between FEC and ARQ decrease by increasing SNR

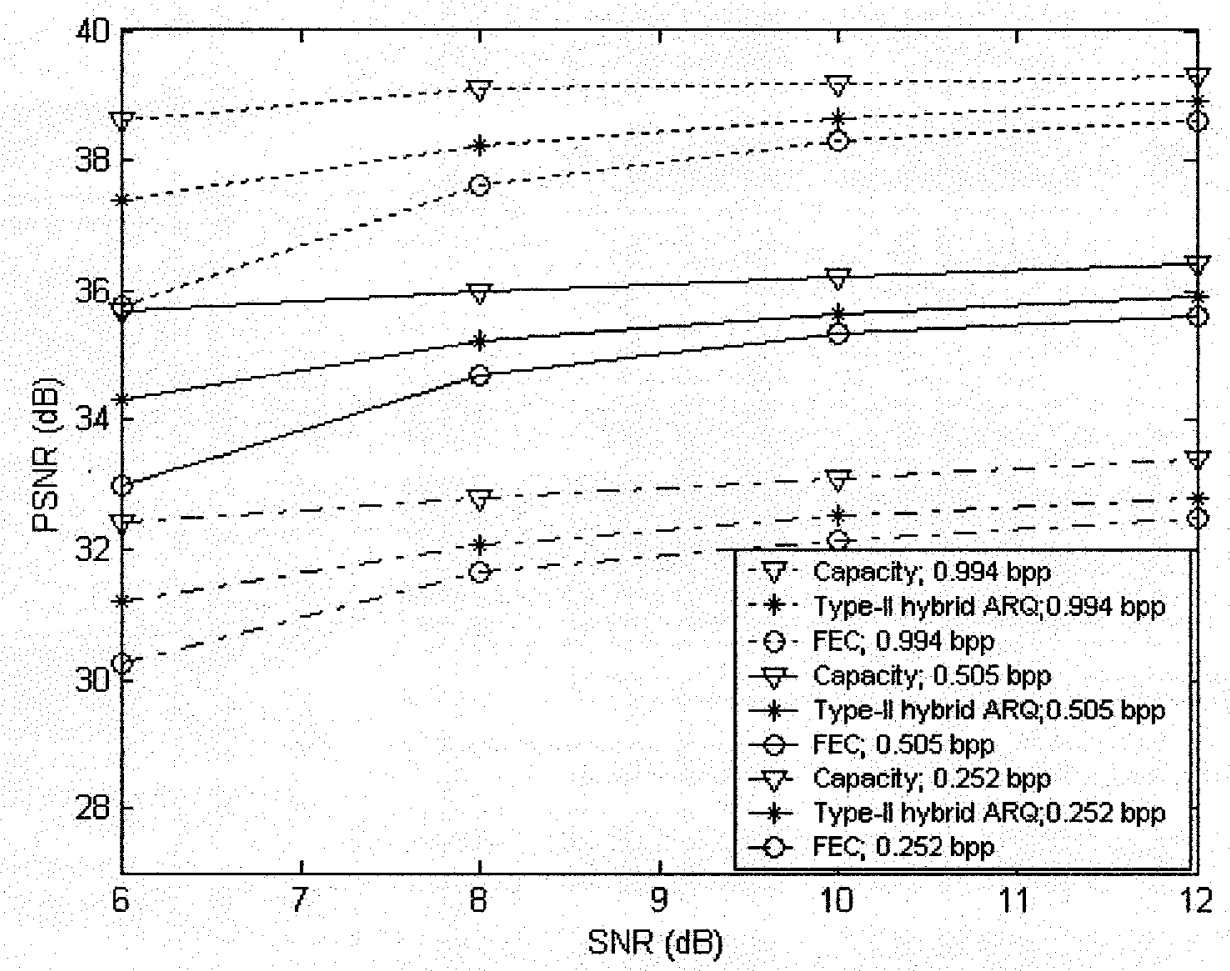

Figure 6.7: The PSNRs of FEC and ARQ schemes for uncorrelated Rayleigh flat-fading channel without SI compared with channel capacity values. 
We also examine the performance of the application of RC-LDPC codes to JPEG2000 coded imaged over correlated Rayleigh fading channels. The channels are modeled with Jakes' simulator [34]. We use the same channel configuration as in Chapter 5 for correlated Rayleigh fading channels: the carrier frequency is set as $900 \mathrm{MHz}$ and the source rate is $9.6 \mathrm{~kb} / \mathrm{s}$. The mobile speeds are $4 \mathrm{~m} / \mathrm{h}, 30 \mathrm{~m} / \mathrm{h}$ and $70 \mathrm{~m} / \mathrm{h}$. Thus, the corresponding normalized Doppler shifts $\left(f_{d}\right)$ are $1.85 \times 10^{-4}, 1.39 \times 10^{-4}$ and $3.24 \times 10^{-3}$ respectively. RC-LDPC codes with the same construction and set of rates as in Chapter 5 are used in the type-II hybrid-ARQ system. Just like the FEC scheme, the PSNRs of the ARQ scheme improve with an increase in channel SNR. The PSNRs are shown in Figure 6.8 (a)-(c). The results for FEC and ARQ are all included for comparison.

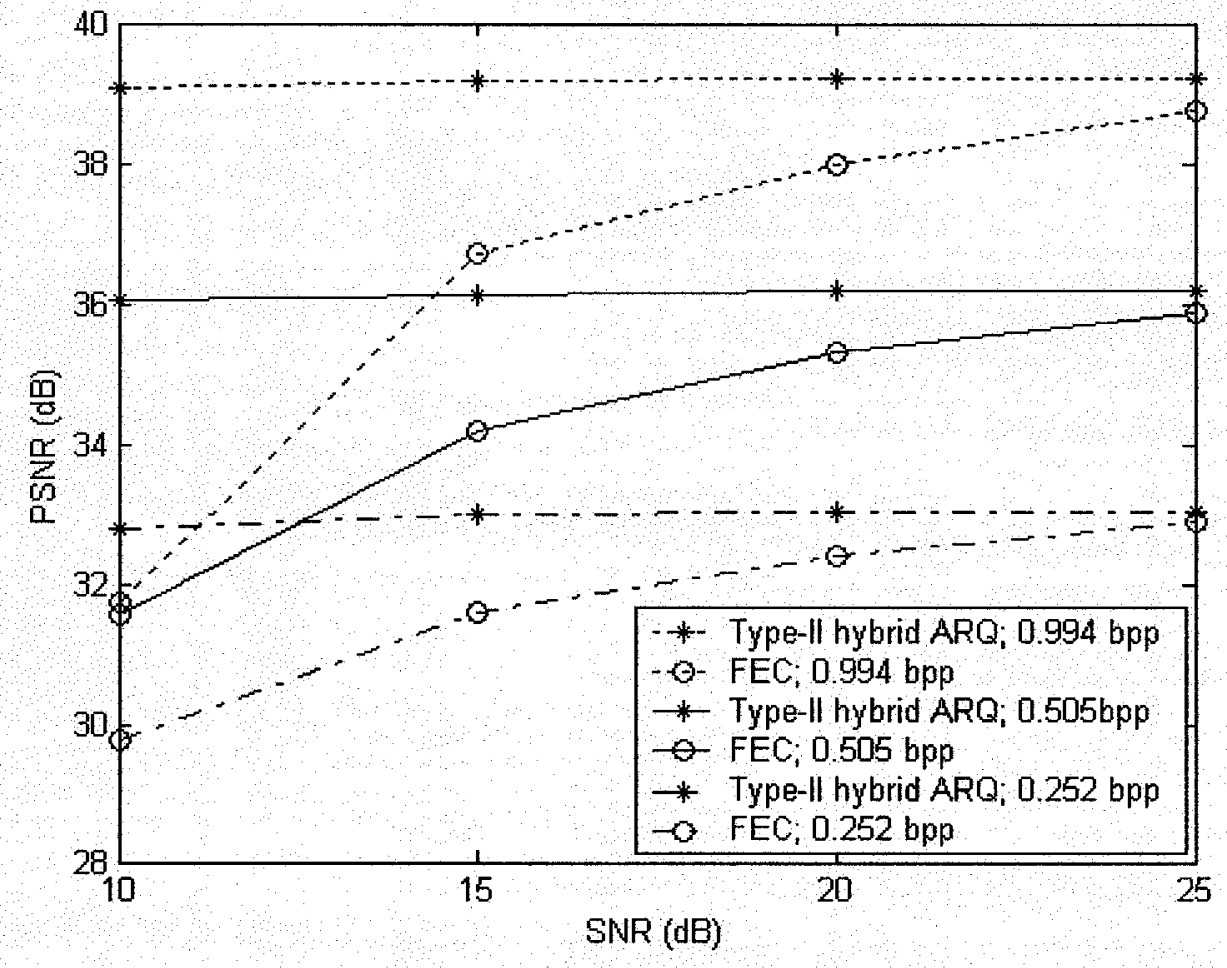

Figure 6.8 (a) $\mathrm{V}=4 \mathrm{~m} / \mathrm{h}$; 


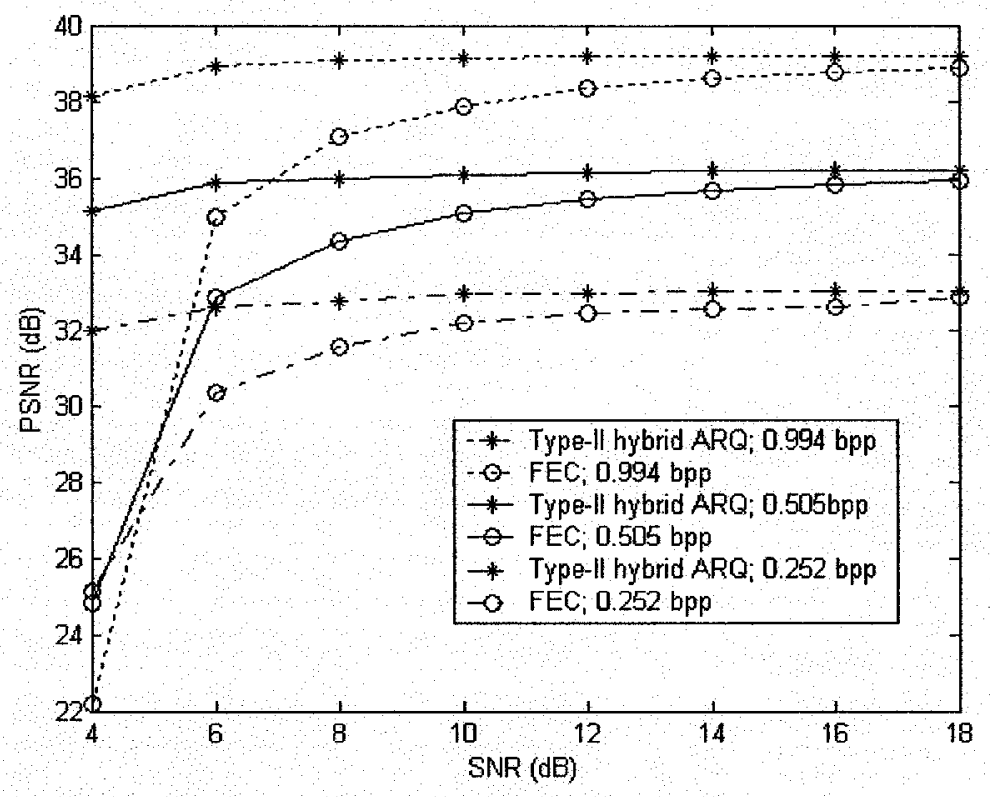

(b) $\mathrm{V}=30 \mathrm{~m} / \mathrm{h}$;

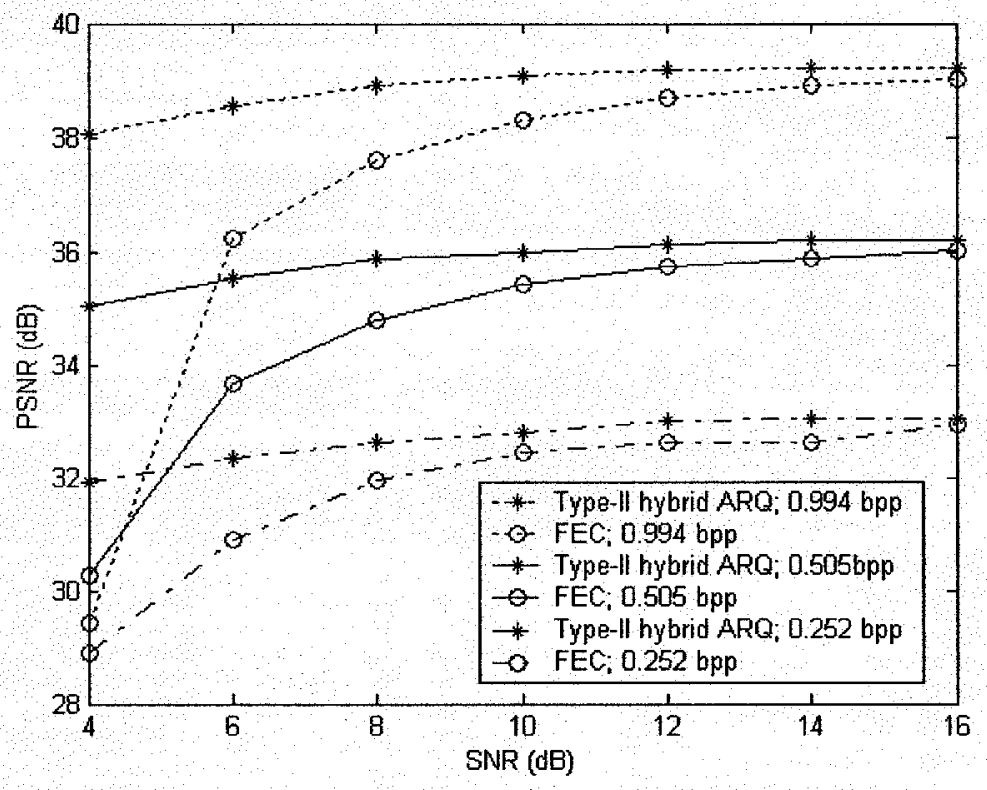

(c) $\mathrm{V}=70 \mathrm{~m} / \mathrm{h}$;

Figure 6.8: The PSNRs of JPEG-2000 coded "Lena" for FEC and ARQ schemes transmitted over correlated Rayleigh fading channels 
Simulation results for uncorrelated/correlated Rayleigh fading channels with/without SI show that type-II hybrid-ARQ scheme is generally superior to FEC scheme in PSNR of the reconstructed image. Big difference between the two schemes appears for low channel SNR. We know that FEC scheme stops decoding the bitstream when the first unrecoverble error happens. The unrecoverble error tends to move to the front of the bitstream with the increase of channel errors. Thus FEC scheme performs worse compared to ARQ scheme at low channel SNRs. 


\section{Chapter 7}

\section{Conclusions and Future Work}

\subsection{Conclusions}

In this thesis, first we proposed a fast trellis-based approach for solving the distortion optimal rate-allocation problem for the robust transmission of progressively coded images over noisy channels based on FEC. The algorithm, which is a faster version of a similar algorithm in [2], provides a speed-up factor of up to about three orders of magnitude for total bit budgets of up to $1 \mathrm{bpp}$. Our simulation results are given for the BSC and are based on the assumption of fixed source packet lengths and common numerator for the channel coding rates. One may, however, obtain similar improvements in speed by applying the same principles to other channels, variable source packet lengths, or channel coding rates with a common denominator.

We also investigated the application of RC-LDPC codes to the transmission of progressively coded images over the BSC and a variety fading chanels. The proposed scheme, which exploits the hierarchial organization of the JP2 bitstream and protects the image using unequal error protection, jointly partitions the bit rates between source and channel codes. The robustness of the scheme has been demonstrated for different images and 
transmission rates, and for a variety of channel conditions. Simulation results show that the proposed scheme outperforms similar joint source/channel coding schemes based on turbo codes and irregular repeat-accumulate codes by up to about $1.1 \mathrm{~dB}$ and $1.0 \mathrm{~dB}$, respectively, and can yield an expected PSNR within about $0.8 \mathrm{~dB}$ of the capacity upper bound. In addition, our RC-LDPC coding scheme is linear-time encodable and can be implemented using only a single pair of encoder/decoder for all the rates. The decoding complexity of the proposed scheme is also comparable to that of the existing scheme based on irregular repeat-accumulate codes and is lower than that of turbo codes. The end-to-end performance of the proposed scheme over fading channels is analyzed by transmitting the progressively coded bitstreams over uncorrelated/correlated Rayleigh fading channels with/without SI. The simulation results that combine SPIHT source coder with RC-LDPC channel codes outperform the existing methods given in [5] and [6]. We also show that our scheme, when JPEG2000 source coder is used, produces PSNRs that are very close to the capacities, with the smallest gap less than $1 \mathrm{~dB}$. We also investigate the influence of channel estimation error on the performance of our system. The robustness of our system could be maintained employing under-estimation at the transmitter. Under-estimation is much less detrimental compared to over-estimation. We also examined this for BSC and find that, in general, underestimation of the channel parameter is more deteriorating than overestimation.

Finally, we studied the type-II hybrid-ARQ based transmission of images over BSC and Rayleigh fading channels. RC-LDPC codes have the potential of achieving nearcapacity throughput. With the ARQ scheme, we obtained PSNRs better than the FEC scheme. The main advantage of type-II hybrid-ARQ image transmission with RC-LDPC 
codes lies in its near-capacity performance. This is promising for applications when delay is a major concern in communications. For the ARQ scheme we haven't compared your results with any of the existing literature (both here and in corresponding sections), because there hasn't been any comparable results.

\subsection{Future Work}

In this thesis, we focused on transmission of still images over noisy channels with JPEG2000/SPIHT and RC-LDPC codes. RC-LDPC codes can be used for volumetric image compression and video sequences. Typically, the video coding algorithm, such as MPEG-4, is also progressively organized within a frame. Research can be done by optimizing RC-LDPC codes such that the PSNR of the video sequences are maximized. Unlike still images, video communication has more strict limitations on the transmission speed. Fast rate-allocation algorithm should be found with the limitation of the transmission speed of the video sequence.

FEC scheme has the characteristic of fast speed. But when the error probability of the channel is high, the performance of the system is not satisfying. ARQ scheme could achieve higher PSNR especially for high channel error probability. But it increases the transmission delay. The initial channel rate could be determined according to the channel condition to reduce the delay. The system will then be more intelligent and flexible according to the channel conditions. There is more work to be done on ARQ and FEC schemes (especially ARQ) in the presence of channel estimation error. 


\section{References}

[1] M. R. Yazdani and A. H. Banihashemi, "On construction of rate-compatible lowdensity parity-check codes," IEEE Comm. Letters, vol. 8, no. 3, pp. 159 - 161, Mar. 2004.

[2] B. A. Banister, B. Belzer, and T. R. Fischer, "Robust image transmission using JPEG2000 and turbo-codes," IEEE Signal Processing Letters, vol. 9, no. 4, pp. 117119, Apr. 2002.

[3] C. Lan, T. Chu, K. R. Narayanan, and Z. Xiong, "Scalable image and video transmission using irregular repeat-accumulation codes with fast algorithm for optimal unequal error protection," IEEE Trans. Comm., vol. 52, no. 7, pp. 1092-1101, Jul. 2004.

[4] A. Said and W. A. Pearlman, "A new fast and efficient image codec based on set partitioning in hierarchical trees," IEEE Trans. Circuits Syst. Video Tech., vol. 6, pp. 243-250, June 1996.

[5] V. Stankovic, R. Hamzaoui, and Z. Xiong, "Efficient channel code rate selection algorithms for forward error correction of packetized multimedia bitstreams in varying channels," IEEE Trans. Multimedia, vol. 6, no. 2, pp. 240-248, Apr. 2004.

[6] D. G. Sachs, R. Anand and K. Ramchandran, "Wireless image transmission using multiple-description based concatenated codes," Proc. SPIE, Image Video Commun. Process., vol 3974, pp. 300-311, Jan. 2000.

[7] D. Taubman and M. Marcellin, JPEG2000: Image Compression Fundamentals, Standards, and Practice. Norwell, MA: Kluwer, 2001. 
[8] JPEG2000

final

committee

draft

[online]:

http://www.jpeg.org/JPEG2000/CDs15444.html.

[9] R. G. Gallager, Low-Density Parity-Check Codes, Cambridge, MA: M.I.T. Press, 1963.

[10] I. M. Tanner, "Recursive approach to low complexity codes," IEEE Trans. Inform. Theory, vol. IT-27, pp. 533-547, Sep. 1981.

[11] S. Y. Chung, G. D. Forney, Jr., T. J. Richardson and R. Urbanke, "On the design of low-density parity check codes within $0.0045 \mathrm{~dB}$ of Shannon limit," IEEE Comm. Lett., vol. 5, no. 2, pp. 58-60, Feb. 2001.

[12] T. J. Richardson and T. L. Urbanke, "The Capacity of Low-Density Parity-Check Codes Under Message-Passing Decoding," IEEE Trans. Inform. Theory, vol. 2, Feb. 2001.

[13] J. Richardson, M. A. Shokrollahi and R. U. Urbanke, "Design of capacityapproaching irregular low-density parity-check codes," IEEE Trans. Inform. Theory, vol. 47, no. 2, pp. $619-637$, Feb. 2001.

[14] X.-Y. Xiao, E. Eleftheriou and D.-M. Arnold, "Progressive edge-growth Tanner graphs," IEEE GLOBECOM, vol. 2 , pp.995 - 1001 , Nov. 2001.

[15] A. Nosratinia, J. Liu, and B. Aazhang, "Source-Channel Rate Allocation for Progressive Transmission of Images," IEEE Trans. Comm., vol. 51, no. 2, pp. 186-196, Feb. 2003. 
[16] J. Kim, R. M. Mersereau, and Y. Altunbasak, "Error-resilient image and video transmission over the Internet using unequal error protection," IEEE Trans. Image Processing, vol. 12, no. 2, pp. 121 - 131, Feb. 2003.

[17] P. G. Sherwood and K. Zeger, "Progressive image coding on noisy channels," in Proc. Data Compression Conf., pp. 72 - 79, March 1997.

[18] V. Sanchez and M. K. Mandal, "Efficient channel protection for JPEG2000 bitstream," IEEE Trans. Circuits and Systems for Video Technology, vol. 14, no. 4, pp. $554-558$, April 2004.

[19] L. Chu, Z. Liu and Z. Xiong, "Joint UEP and layered source coding with application to transmission of JPEG-2000 coded images," IEEE GLOBCOM, vol. 3, pp. 20362039, Nov. 2001.

[20] V. Sanchez and M. K. Mandal, "Robust transmission of JPEG2000 images over noisy channels," IEEE Trans. On Consumer Electronics, vol. 48, no. 3, pp. 451-456, Aug. 2002.

[21] Y. Mao and A. H. Banihashemi, "A heuristic search for good low-density paritycheck codes ar short block length," Proc of IEEE Int. Conf. on Comm. (ICC), vol. 1, pp. 41-44, 2001.

[22] R. Hamzaoui, V. Stankovic, and Z. Xiong, "Rate-based versus distortion-based optimal joint source-channel coding," in Proc. DCC, Snowbird, UT, pp.63-67, Apr. 2002. 
[23] V. Chande, and N. Farvardin, "Progressive transmission of images over memoryless noisy channels," IEEE Journal and Selected Areas in Comm., vol. 18, no. 6, pp. 850-860, Jun. 2000.

[24] X. Pan, A. Cuhadar and A. H. Banihashemi, "Joint Source/Channel Coding with JPEG2000 and Rate-Compatible Low-Density Parity-Check (RC-LDPC) Codes," submitted to IEEE Trans. Signal Processing, July 2004.

[25] A.Amraoui, http://lthcwww.epfl.ch/research/ldpcopt.

[26] http://vision.ploy.edu:8080/ -zhul/EL512/SampleData.html.

[27] Kakadu software implementation of JPEG2000 standard: [online]http://www.kakadusoftware.com/.

[28] D. S. Taubman and Michael W. Marcellin, JPEG2000 image compression fundamentals, standards, and practice, Norwell: Kluwer Academic Publishers, 2002.

[29] P. G. Sherwood and K. Zeger, "Error protection for progressive image transmission over memoryless and fading channels," IEEE Trans. Comm., vol. 46, no. 12, Dec. 1998.

[30] P. C. Cosman, J. K. Rogers, P. G. Sherwood, and K. Zeger, "Combined forward error control and packetized zerotree wavelet encoding for transmission of images over varying channels," IEEE Trans. Image Processing, vol. 9, no. 6, pp. 982-993, Jun. 2000.

[31] M. Grangetto, E. Magli and G. Olmo, "Ensuring quality of service for image transmission: hybrid loss protection," IEEE Trans. Image Processing, vol. 13, no. 6, pp. 751-757, Jun. 2004. 
[32] V. Stankovic, R. Hamzaoui, and Z. Xiong, "Real-time error protection of embedded codes for packet erasure and fading channels," IEEE Trans. Circuits and Systems for Video Technology, vol. 14, no. 8, pp. 1064-1072, Aug. 2004.

[33] J. Hou, P. H. Siegel, and L.B. Milstein, "Performance analysis and code optimization of low-density parity-check codes on Rayleigh fading channels," IEEE Journal on Selected Areas in Comm., vol. 19, no. 5, pp. 924-934, May 2001.

[34] W. C. Jakes, Microwave Mobile Communications. New York: Wiley, 1974.

[35] T. F. Wong, "Numerical calculation of systemetric capacity of Rayleigh fading channel with BPSK/QPSK," IEEE Comm. Letters, vol. 5, no. 5, pp. 328-330, Aug. 2001.

[36] I. C. Abou-Faycal, M. D. Trott, and S. Shamai, "The capacity of discrete-time memoryless Rayleigh-fading channels," IEEE Trans. Inform. Theory, vol. 47, no. 4, pp. 1290-1301, May 2001. 